\title{
Characterization of dense Planck clumps observed with Herschel and SCUBA-2 $2^{\star}, \star \star$
}

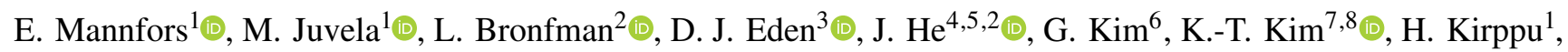

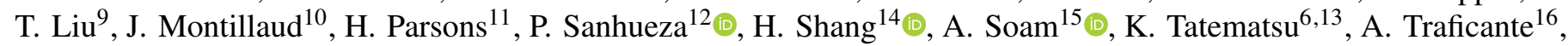 \\ M. S. Väisälä ${ }^{14}\left(0\right.$, and C. W. Lee ${ }^{7,8}(0)$ \\ (Affiliations can be found after the references)
}

Received 21 February 2020 / Accepted 23 May 2021

\begin{abstract}
Context. Although the basic processes of star formation (SF) are known, more research is needed on SF across multiple scales and environments. The Planck all-sky survey provided a large catalog of Galactic cold clouds and clumps that have been the target of several follow-up surveys. Aims. We aim to characterize a diverse selection of dense, potentially star-forming cores, clumps, and clouds within the Milky Way in terms of their dust emission and SF activity.

Methods. We studied 53 fields that have been observed in the JCMT SCUBA-2 continuum survey SCOPE and have been mapped with Herschel. We estimated dust properties by fitting Herschel observations with modified blackbody functions, studied the relationship between dust temperature and dust opacity spectral index $\beta$, and estimated column densities. We extracted clumps from the SCUBA-2 $850 \mu \mathrm{m}$ maps with the FellWalker algorithm and examined their masses and sizes. Clumps are associated with young stellar objects found in several catalogs. We estimated the gravitational stability of the clumps with virial analysis. The clumps are categorized as unbound starless, prestellar, or protostellar.

Results. We find 529 dense clumps, typically with high column densities from $(0.3-4.8) \times 10^{22} \mathrm{~cm}^{-2}$, with a mean of $(1.5 \pm 0.04) \times 10^{22} \mathrm{~cm}{ }^{-2}$, low temperatures $(T \sim 10-20 \mathrm{~K})$, and estimated submillimeter $\beta=1.7 \pm 0.1$. We detect a slight increase in opacity spectral index toward millimeter wavelengths. Masses of the sources range from $0.04 M_{\odot}$ to $4259 M_{\odot}$. Mass, linear size, and temperature are correlated with distance. Furthermore, the estimated gravitational stability is dependent on distance, and more distant clumps appear more virially bound. Finally, we present a catalog of properties of the clumps.

Conclusions. Our sources present a large array of SF regions, from high-latitude, nearby diffuse clouds to large SF complexes near the Galactic center. Analysis of these regions will continue with the addition of molecular line data, which will allow us to study the densest regions of the clumps in more detail.
\end{abstract}

Key words. methods: observational - stars: formation - ISM: clouds - dust, extinction - infrared: ISM - ISM: general

\section{Introduction}

Star formation (SF) processes involve all scales within the interstellar medium (ISM). Large filaments in molecular clouds (MCs) fragment into parsec-scale clumps and subparsec cores, which can eventually form young stellar objects (YSOs; Offner et al. 2014). SF processes are affected by the combined effects of turbulence, magnetism, thermal pressure, and gravity. There is evidence that the relative importance of these forces changes in structures from filaments to cores (Mattern et al. 2018; Liu et al. 2018b; Tang et al. 2019; Soam et al. 2019; Traficante et al. 2020). In addition, there is evidence that larger mass reservoirs are required for SF in energetic environments such as the Galactic center (Trujillo-Gomez et al. 2019). Regions with second-generation SF, such as the edges of bubbles, are also likely to have higher column and volume density than the general ISM (Thompson et al. 2012; Liu et al. 2012, 2016;

\footnotetext{
* Planck (http://www. esa.int/Planck) is a project of the European Space Agency - ESA - with instruments provided by two scientific consortia funded by ESA member states (in particular the lead countries: France and Italy) with contributions from NASA (USA), and telescope reflectors provided in a collaboration between ESA and a scientific Consortium led and funded by Denmark.

$\star \star$ Herschel is an ESA space observatory with science instruments provided by European-led Principal Investigator consortia and with important participation from NASA.
}

Li et al. 2019b), leading to an increased occurrence of SF in these regions (Kendrew et al. 2012; Palmeirim et al. 2017). Changing environments make it necessary to study the interplay of these forces under different conditions. Regions of SF within the Milky Way provide an opportunity to study SF from small highlatitude clouds to the dense MCs within the Galaxy's spiral arms. We seek to extend the range of SF research by studying potentially star-forming clouds up to $4.5 \mathrm{kpc}$ in distance, a sample that includes high-mass SF regions.

Filamentary structures are believed to be ubiquitous within the ISM (e.g., Men'shchikov et al. 2010; André et al. 2010, 2014; Arzoumanian et al. 2011; Hennemann et al. 2012; Juvela et al. 2012; Malinen et al. 2012; Palmeirim et al. 2013; Wang et al. 2015). Fragmentation creates clumpy condensations of radii under $2 \mathrm{pc}$ as well as smaller cores of radii under $0.5 \mathrm{pc}$ (Offner et al. 2014; Wang et al. 2014; Zhang et al. 2015). Whether a core will form stars in the future depends on the relative strength of gravity, thermal pressure, turbulence, and magnetic energy.

Nonthermal motions inside large MCs are supersonic (Larson 2003), and kinetic and magnetic energy dominate at large scales (Li et al. 2015; Pattle et al. 2017a). The magnetic field dominates in the difuse ISM (Heiles \& Troland 2005; Basu \& Roy 2013), while the effect of gravity and external pressure is significant on smaller scales (McKee \& Ostriker 2007; Maruta et al. 2010; Pattle et al. 2015). Magnetic energy can be crucial to the formation of filaments, with filaments being either 
contained by magnetic flux or contracting along magnetic field lines (Crutcher 2012; Juvela et al. 2018a; Gholipour 2019; Tang et al. 2019; Soam et al. 2019). Fragmentation at core scales is likely rapid enough that it does not depend on the larger environment (Kainulainen et al. 2013; Hacar et al. 2013; Teixeira et al. 2016; Mattern et al. 2018). In clouds that have already begun SF, feedback from protostars is effective at diminishing future SF, and at lower densities, destroying the parent cloud (Ngoumou et al. 2015; Tasker et al. 2015; Fontani et al. 2018). The relative strengths of these forces depend on the scale at which they are observed and on the surrounding environment. To understand these various forces, it is necessary to incorporate multiple scales of SF into our research.

Cores that show signs of YSOs already are classified as protostellar (PS). Of the starless cores, those that are gravitationally bound and will likely form stars in the future are classified as prestellar (PRE), and those that are too diffuse for gravitational collapse as unbound starless (SL). In cases in which we refer to all starless cores, we use the acronym SL PRE. In time, unbound starless cores disperse unless they lose the internal support against collapse or more mass is accreted onto them. We seek to answer if the star-forming status of dense clumps can be predicted from continuum data, by observing the properties available through continuum observations: temperatures, column densities, and masses.

Objects within the cold ISM are studied by observing dust and molecular lines; the latter method is important to the study of cloud stability and to that of general kinematics (Motte et al. 1998; Andre et al. 2000; Enoch et al. 2007; Pattle et al. 2017b). The spectral energy distribution (SED) of an accreting protostar peaks at under $100 \mu \mathrm{m}$ (Benedettini et al. 2018), and thus emission in far-infrared (IR) wavelengths can be used to determine the presence of YSOs. At wavelengths of over $100 \mu \mathrm{m}$, dust emission from the cold, dense ISM is detectable, which is useful for estimating the temperature and density of the clouds. This emission can be approximated by modified blackbody (MBB) column emission (Sadavoy et al. 2013; Planck Collaboration XI 2014), which gives the opportunity to estimate the temperature of the emitting grains. According to theory, at far-IR and submillimeter wavelengths opacity spectral index $\beta$ should be mostly independent of temperature and restricted to the values of 1-2 (Bohren \& Huffman 1998). However, a dependence of spectral index on temperature was first detected by Dupac et al. (2003), and spectral index has been found to have values above 2 in cold regions (Juvela et al. 2015). Recent $\mathrm{cm}$ observations of Orion have shown significant mm excess compared to shorter wavelengths, lowering the estimated value of the opacity spectral index (Mason et al. 2020). Uncertainty on the grain sizes and optical properties of the cold dust still exists. Understanding the behavior of this parameter is important because the value of the spectral index affects the shape of the MBB function and thus the derived dust properties.

The European Space Agency (ESA) Herschel space observatory has been crucial to observing the cold ISM. Key surveys using Herschel include Hi-GAL (the Herschel infrared Galactic Plane Survey) (PI: S. Molinari), which mapped the inner Galactic plane (Molinari et al. 2010) and the Gould Belt Survey (PI: P. André), which imaged major SF regions within $500 \mathrm{pc}$ of the Sun in clouds within the Gould Belt (Ward-Thompson et al. 2007; André et al. 2010). The Gould Belt Survey has resulted in multiple studies about nearby low-mass SF regions (e.g., Sadavoy et al. 2013; Könyves et al. 2015; Pattle et al. 2015, 2017b; Bresnahan et al. 2018; Benedettini et al. 2018). Cold, dense Galactic regions have been observed as part of the
Galactic cold cores (GCC) survey (PI: M. Juvela; Planck Collaboration XXIII 2011; Planck Collaboration XXII 2011; Planck Collaboration XXVIII 2016) (see Sect. 2), using fields selected from the Planck Galactic Cold Clumps (PGCC) catalog (Planck Collaboration XXIII 2011; Planck Collaboration XXII 2011; Planck Collaboration XXVIII 2016).

Many PGCC sources have been imaged with ground-based observatories, as in the SCOPE (SCUBA-2 Continuum Observations of Pre-protostellar Evolution) survey (PI T. Liu), which used the SCUBA-2 (Submillimetre Common-User Bolometer Array 2) instrument (Holland et al. 2013) on the James Clerk Maxwell Telescope (JCMT; see Sect. 2). Preliminary analysis of SCUBA-2 sources combined with Herschel observations has been performed (Juvela et al. 2018b; Liu et al. 2018a) and a catalog of detected clumps has recently been published (Eden et al. 2019).

In this paper, we analyze 53 high-column density fields from the SCOPE survey that have also been mapped with Herschel. With this sample we seek to understand SF by analyzing starforming regions in a wide range of Galactic environments. We combine Herschel data with JCMT SCUBA-2 data to detect clumps and to study the dust properties of these dense environments and their SF status. This study of the properties of clumps, their dust properties, and SF activity also serves as preparation for future investigations where the chemical properties of the clumps will be studied.

This paper is structured as follows: Sect. 2 describes the observation and reduction of these data and Sect. 3 the methodology used in analysis. Section 4 presents the results of analysis of the dust properties and of the dense clumps, which are further discussed in Sect. 5.

\section{Observations}

In this paper, we use Herschel and JCMT dust continuum observations to study high-column-density clumps in 53 fields. Around half of these fields also have Taeduk Radio Astronomy Observatory (TRAO) $)^{1} \mathrm{CO}$ line data. These regions were originally selected from the PGCC catalog and are also the target of future chemical studies. We present instrumental characteristics for all bands used in Appendix A.

\subsection{Target selection}

The PGCC has led to several follow-up projects, including the TOP (TRAO Observations of Planck cold clumps; PI: T. Liu) survey, which aims to observe 2000 sources that have high column densities with TRAO in ${ }^{12} \mathrm{CO}$ and ${ }^{13} \mathrm{CO} J=1-0$ transitions. Preference was given to sources that had also been imaged with Herschel, but the objects are located across all Galactic latitudes and longitudes. From the TOP sources, 1235 PGCCs with high column densities, generally over $10^{21} \mathrm{~cm}^{-2}$ in Planck measurements, were imaged with the JCMT on Mauna Kea in Hawai' $i$ in $850 \mu \mathrm{m}$ continuum emission for the SCOPE survey (Liu et al. 2018a; Eden et al. 2019). For a complete sample, some PGCCs with lower column densities at high Galactic latitude were also imaged. The purpose of the TOP-SCOPE joint survey is to study the formation and evolution of dense clumps and filaments in a variety of environments (Liu et al. 2018a). The regions are located across the whole sky, although mostly concentrated around the Galactic plane (see Fig. 1).

\footnotetext{
https://radio.kasi.re.kr/trao/main_trao.php
} 


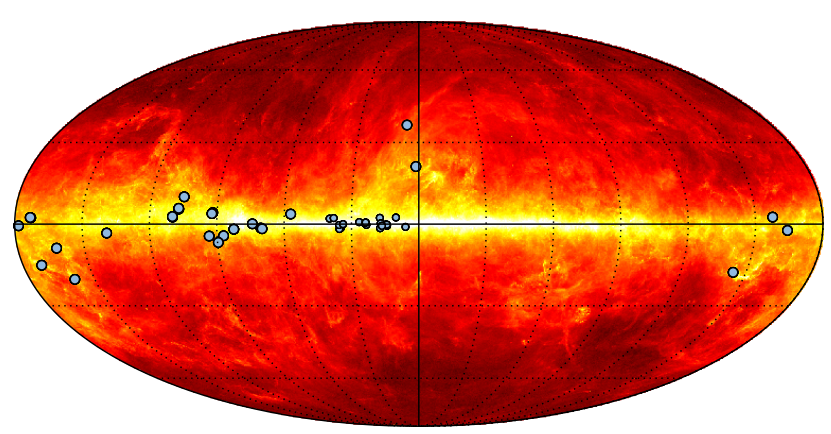

Fig. 1. Fifty-three fields denoted on the $857 \mathrm{GHz}$ Planck surface brightness map of the Galaxy. Some SCUBA-2 fields are located on the same Herschel field, and the markers overlap at this scale.

These fields are also being observed with the Nobeyama $45 \mathrm{~m}$ radio telescope (PI: Ken'ichi Tatematsu) in $\mathrm{DNC}, \mathrm{HN}^{13} \mathrm{C}$, $\mathrm{N}_{2} \mathrm{D}^{+}, \mathrm{N}_{2} \mathrm{H}^{+}, \mathrm{CCS}, \mathrm{HC}_{3} \mathrm{~N}$, and cyclic- $\mathrm{C}_{3} \mathrm{H}_{2}$. The purpose of the Nobeyama survey is to characterize the chemical evolution of the cores and find the earliest signs of SF, as in Tatematsu et al. (2017). The densest clumps in these 53 fields have also been observed in $\mathrm{NH}_{3}$ lines with the Effelsberg $100 \mathrm{~m}$ radio telescope (PI: Viktor Tóth). These molecular line observations are discussed in Tatematsu et al. (2020) and Kim et al. (2020).

\subsection{SCUBA-2 observations}

The SCUBA-2 $850 \mu \mathrm{m}$ observations were made as part of the SCOPE program (Liu et al. 2018a, ; project ID: M16AL003). As the average angular size of PGCC sources is $8^{\prime}$ (Liu et al. 2018a), the fields were observed with the constant velocity (CV) daisy scan pattern, which is suitable for sources up to $12^{\prime}$ in size (Bintley et al. 2014) ${ }^{2}$. The average observing time was 16 minutes per field (Liu et al. 2018a). Data reduction was performed using an iterative mapmaking technique described in Chapin et al. (2013). This method compares detector beams and removes any lowfrequency signals that are correlated between the beams, which removes contamination from the atmosphere but also any largescale structure above 200" (Liu et al. 2018a). In the pipeline, a mean flux conversion factor of $554 \mathrm{Jy} \mathrm{pW}^{-1}$ beam $^{-1}$ was used to convert data from pW to Jy beam ${ }^{-1}$ by Liu et al. (2018a). SCUBA-2 data show compact sources with high column densities and can thus be used to find dense cores in the fields. Owing to its resolution of $\sim 14^{\prime \prime}$, SCUBA-2 can detect dense cores with size of $\sim 0.1 \mathrm{pc}$ to within $2 \mathrm{kpc}$. As noise ranged from $6-$ $10 \mathrm{mJy}^{-1}$ beam $^{-1}$ in the central $3^{\prime}$ to $10-30 \mathrm{mJy} \mathrm{beam}^{-1}$ out to $12^{\prime \prime}$ (Liu et al. 2018a), we assume an uncertainty of $10 \mathrm{mJy} \mathrm{beam}^{-1}$ for the SCUBA-2 data, as in Juvela et al. (2018b).

\subsection{Herschel observations}

We used data observed with two instruments on ESA's Herschel space observatory (Pilbratt et al. 2010): SPIRE (Spectral and Photometric Imaging Receiver; Griffin et al. 2010) and PACS (Photodetector Array Camera and Spectrometer; Poglitsch et al. 2010). The 250,350 , and $500 \mu \mathrm{m}$ data were imaged simultaneously using the SPIRE photometer and the 70,100, and $160 \mu \mathrm{m}$ data using PACS. The PACS imaged either 70 and $160 \mu \mathrm{m}$ or 100 and $160 \mu \mathrm{m}$ maps simultaneously.

\footnotetext{
2 https://www.eaobservatory.org/jcmt/instrumentation/ continuum/scuba-2/observing-modes/
}
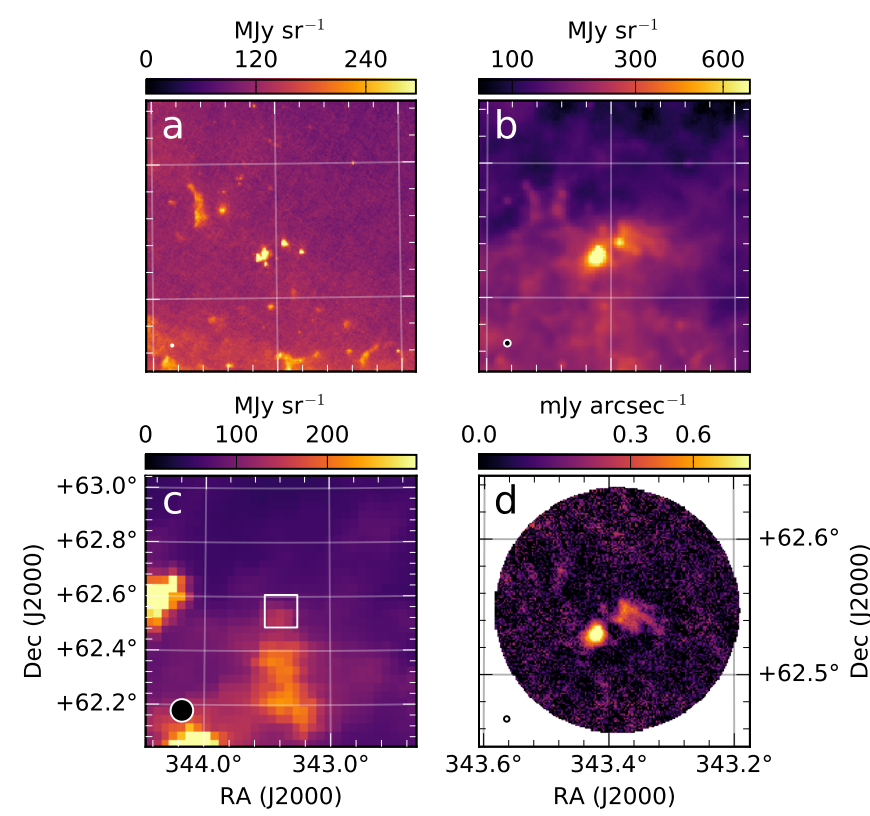

Fig. 2. Surface brightness maps of field G109.81+02.72, part of the Cepheus MC, in PACS $70 \mu \mathrm{m}(a)$, SPIRE $250 \mu \mathrm{m}($ b), Planck $857 \mathrm{GHz}$ $(\sim 350 \mu \mathrm{m})(c)$, and SCUBA-2 $850 \mu \mathrm{m}(d)$. SCUBA-2 data are represented in units of $\mathrm{mJy} \mathrm{arcsec}^{-2}$, all others are in $\mathrm{MJy} \mathrm{sr}^{-1}$. The Planck image is $1 \times 1$ degrees in size. All other images cover a smaller area of $0.2 \times 0.2$ degrees, as indicated by the rectangle drawn on the Planck image. The beam is shown in the lower left corner of each frame.

Our sample consists of 53 TOP-SCOPE fields, 45 of which have both Herschel PACS $160 \mu \mathrm{m}$ and SPIRE data, 3 only SPIRE data, and 1 only PACS $160 \mu \mathrm{m}$ data. In addition, 29 fields have PACS $70 \mu \mathrm{m}$ data and 25 PACS $100 \mu \mathrm{m}$ data. The observations are summarized in Tabare summarized in Table C.1. Angular resolution of the Herschel instruments ranges from 5.6" at $70 \mu \mathrm{m}$ to $35.4^{\prime \prime}$ at $500 \mu \mathrm{m}$. Unlike the SPIRE fields used, the PACS fields do not have a constant FWHM size due to different scanning velocities; a faster scan results in elongation of the effective beam.

Herschel data were collected from the Herschel Science Archive $^{3}$. The data were reduced by the official pipeline to level 2.5 or 3 , and the SPIRE maps include intensity zero-point corrections, which are obtained from comparison to Planck measurements. The PACS images are not zero point corrected by the pipeline. All data were converted to units of $\mathrm{MJy} \mathrm{sr}^{-1}$.

Extended emission is visible in the SPIRE observations (Fig. 2b), which are useful for determining cloud characteristics such as temperature and column density. Hotter protostellar sources are visible at shorter wavelengths, and 70 or $100 \mu \mathrm{m}$ detection are a strong indicator of ongoing SF. Figure 2a shows an example of several protostellar sources at $70 \mu \mathrm{m}$, which are forming inside the clumps visible in $850 \mu \mathrm{m}$ (Fig. $2 \mathrm{~d}$ ).

\subsection{TRAO observations}

The TRAO data were observed as part of the TOP key science program (PI: T. Liu), which observed 2000 PGCCs in ${ }^{12} \mathrm{CO}(J=1-0)$ and ${ }^{13} \mathrm{CO}(J=1-0)$, as well as $\sim 10 \%$ of these fields in $\mathrm{C}^{18} \mathrm{O}(J=1-0)$. The ${ }^{12} \mathrm{CO}$ and ${ }^{13} \mathrm{CO}$ lines were mapped simultaneously using on-the-fly mapping, with maps being $\leq 30^{\prime} \times 30^{\prime}$ in size. These data were regridded to a pixel

\footnotetext{
http://archives.esac.esa.int/hsa/whsa/
} 
size of $24^{\prime \prime}$ and smoothed to $0.33 \mathrm{~km} \mathrm{~s}^{-1}$, and the baseline was removed with a first- or third-order polynomial. The front end of the TRAO telescope is the SEcond QUabbin Optical Image Array (SEQUOIA-TRAO), a $4 \times 4$ array receiver with spatial separation of $89^{\prime \prime}$, and the back end a fast Fourier transform spectrometer with $4096 \times 2$ channels at a resolution of $15 \mathrm{kHz}$ (Chung et al. 2019). Full TRAO data reduction steps are available in Liu et al. (2018a), but we have listed instrumental specifics in Table A. 2 for ${ }^{12} \mathrm{CO}$ and ${ }^{13} \mathrm{CO}$ observations.

\section{Methods}

\subsection{Distance estimates}

In addition to the distances from literature, we calculated distances to 27 fields based on ${ }^{12} \mathrm{CO}$ TRAO line data, using a kinematic distance code written by Chris Beaumont ${ }^{4}$ and the rotation curve of Brand \& Blitz (1993). This method gives two solutions for heliocentric distance $d ; d_{\min }-\Delta d, d_{\min }+\Delta d$, where

$d_{\min }=R_{0} \cos (l)$

$\Delta d=\sqrt{R^{2}-\left(R_{0} \sin (l)\right)^{2}}$,

using $R_{0}=8.8 \mathrm{kpc}$. One realistic positive solution exists for objects outside the solar circle and two solutions for objects inside of the solar circle (Russeil et al. 2011). For sources inside the solar circle, we choose the lower solution for a field's distance. The derived galacto- and heliocentric distances for these fields are listed in Table D.1. For fields that have distances estimated in the literature using other methods other than than kinematic estimates, we use those distances instead owing to the uncertain nature of kinematic distances.

\subsection{Dust temperature and column density maps}

To estimate temperature, column density, optical depth, and intensity of the fields, the observed data were fitted with a MBB function. The MBB takes the form

$F_{v}=F_{v_{0}} \frac{B_{v}(T)}{B_{v_{0}}(T)}\left(\frac{v}{v_{0}}\right)^{\beta}$,

where $F_{v}$ is the flux density at a frequency $v$ and $F_{v_{0}}$ the flux density at a reference frequency $v_{0}=250 \mu \mathrm{m}$. When using a fixed value for the opacity spectral index, we fixed it to $\beta=1.8$, which should be appropriate for dense regions (Juvela et al. 2015).

Unless otherwise stated, analysis of dense clumps used only SPIRE bands. Before fitting the MBB, the maps of the three wavelengths were convolved to a $35.4^{\prime \prime}$ resolution using a Gaussian beam with beam sizes listed in Appendix A and reprojected onto the same pixels. We did not use SCUBA-2 data in the MBB fitting of maps because, as a result of filtering, these do not contain extended emission. The SCUBA-2 measurements were used only in clump analysis, where we compared the fluxes to MBB fits made to the Herschel data. Shorter wavelength PACS data are not used for analysis of clumps because PACS data exist only for 45 out of the 53 fields. The shorter wavelengths are sensitive to temperature variations and therefore their inclusion could also increase bias in the derived physical quantities (i.e., lead to higher dust temperature and lower column density estimates) (Shetty et al. 2009a; Juvela \& Ysard 2012). We compare

\footnotetext{
4 http://www.ifa.hawaii.edu/users/beaumont/code/ kdist-code.html
}

the results of SED fits including PACS and SCUBA-2 data with those that only use SPIRE data in Appendix B.

A least-squares fit of the MBB function was performed on each pixel. Based on the fits, we made maps of the dust color temperature $T$, intensity $I_{250}$, and the optical depth $\tau_{250}$ at $250 \mu \mathrm{m}$. This intensity map is referred to as the $I_{250}$ map, whereas the original observed $250 \mu \mathrm{m}$ map is referred to as the SPIRE $250 \mu \mathrm{m}$ map. The $I_{250}$ map is the best fit of the MBB at each pixel. In the future we refer to dust color temperature simply as temperature. Optical depth at $250 \mu \mathrm{m}$ was calculated by

$\tau_{250}=I_{250} / B_{250}(T)$,

where $B_{250}(T)$ is the value of the Planck function at $250 \mu \mathrm{m}$. Column density $N\left(\mathrm{H}_{2}\right)$ was estimated using

$N\left(\mathrm{H}_{2}\right)=\tau_{250} / \kappa_{250} / \mu_{\mathrm{H}_{2}}$,

where $\mu_{\mathrm{H}_{2}}=2.8 m_{\mathrm{H}}$ is the total mass relative to the $\mathrm{H}_{2}$ molecule. We assume dust opacity $\kappa_{v}$ of Beckwith et al. (1990), which takes into account the gas-to-dust mass ratio

$\kappa_{v}=0.1(v / 1000 \mathrm{GHz})^{\beta} \mathrm{cm}^{2} \mathrm{~g}^{-1}$,

using $\beta=1.8$. Optical depth and column density are quantities averaged over the effective beam, $35.4^{\prime \prime}$.

\subsection{Clump detection}

The FellWalker algorithm from the Starlink: FindClumps ${ }^{5}$ package was used to extract clumps from SCUBA-2 $850 \mu \mathrm{m}$ maps. FellWalker is a watershed algorithm, which uses uphill paths rather than contours to define the clumps (Berry 2015). FellWalker has been used in other SCUBA-2 surveys (e.g., Rumble et al. 2015; Eden et al. 2017, 2019; Johnstone et al. 2017; Juvela et al. 2018b), as well as in, for example, Zhang et al. (2018), using Herschel column density maps. The input values used in the algorithm are presented in Appendix E. The RMS noise values are calculated from the reference regions (Table F.1, Appendix F). FellWalker, along with ClumpFind and several other algorithms, are included in Starlink's CUPID-package. We compare the performance of some of these algorithms in Appendix G.

\subsection{Clump properties}

Clump properties were extracted using Starlink's EXTRACTCLUMPS algorithm from the CUPID package using $850 \mu \mathrm{m}$ data as well. Up to 15 clump properties were derived, either directly with EXTRACTCLUMPS or extracted from Herschel maps using those parameters. The characteristics are listed in Table 1. The $850 \mu \mathrm{m}$ clump maps are at the resolution of the SCUBA-2 data, 14.6", and the temperature and column density maps at the resolution of the SPIRE $500 \mu \mathrm{m}$ data, 35.4".

Central coordinates, position angle, and angular sizes of the major and minor axes of the clump are given by the EXTRACTCLUMPS routine. Average values of dust temperature $\langle T\rangle$, surface brightness $\left\langle I_{250}\right\rangle$, and column density $\left\langle N\left(\mathrm{H}_{2}\right)\right\rangle$ over the clump area were calculated using the Herschel SPIRE maps. The source flux density $F_{v}$ is calculated by multiplying the average $850 \mu \mathrm{m}$ surface brightness with the clump solid angle, $F_{850}=\left\langle I_{850}\right\rangle \Omega$. Volume density is estimated by dividing mass by

\footnotetext{
5 http://starlink. eao.hawaii.edu/docs/sun255.htx/ sun255ss 5. html
} 
Table 1. Characteristics calculated for each clump.

\begin{tabular}{lc}
\hline \hline Coordinates & Position angle \\
Angular size & Effective radius $R_{\text {eff }}$ \\
Flux density $F_{v}$ & Temperature $T$ \\
Intensity at $250 \mu \mathrm{m} I_{250}$ & Mean column density $N\left(\mathrm{H}_{2}\right)$ \\
Mass $M$ & Peak column density $N\left(\mathrm{H}_{2}\right)_{850}$ \\
Virial mass $M_{\text {vir }}$ & Virial alpha $\alpha_{\text {vir }}$ \\
Number of YSOs $N_{\text {YSO }}$ & Number of confirmed YSOs $N_{\text {YSO, } \Delta \mathrm{C}}$ \\
Clump type & \\
\hline
\end{tabular}

Notes. Mean column density is calculated from Herschel SPIRE data and peak column density from SCUBA-2 data. See Sect. 3.6.3 for explanation on the difference between $N_{\mathrm{YsO}}$ and $N_{\mathrm{YSO}, \Delta \mathrm{C}}$.

the clump volume, mean molecular weight $\mu$, and atomic hydrogen mass $m_{\mathrm{H}_{2}}$ (Liu et al. 2018a) as follows:

$n=\frac{M}{\frac{4}{3} \pi R_{\mathrm{eff}}^{3} \mu \mathrm{m}_{\mathrm{H}_{2}}}$.

Radius and mass are calculated as in Liu et al. (2018a). Effective radius is written as

$R_{\mathrm{eff}}=\sqrt{a b}$,

where $a$ and $b$ are the deconvolved spatial major and minor axis FWHMs, which are given in arcsec by EXTRACTCLUMPS. Masses of clumps were calculated from Eq. (1) as follows:

$M=\frac{F_{\nu} d^{2}}{\kappa_{\nu} B_{v}(T)}$,

where $F_{v}$ is the flux density of the clump, $d$ the distance to the field, $\kappa_{v}$ the dust opacity, and $B_{v}(T)$ is the Planck function at the clump average dust temperature, setting the temperature of any clumps without temperature estimates or with $T<10 \mathrm{~K}$ to the mean overall clump temperature.

\subsection{Gravitational stability}

We estimate gravitational boundedness for all clumps with mass estimates, assuming that they are isolated systems supported against gravity by thermal pressure and turbulence. The level of turbulence within the clouds is estimated using ${ }^{13} \mathrm{CO}$ line width measurements from Wang et al. (2018). Velocity dispersion $\sigma_{\text {tot }}$ is calculated from the full width at half maximum (FWHM) line width $\Delta V$ of the ${ }^{13} \mathrm{CO}$ molecule and is written as

$\sigma_{\text {tot }}=\sqrt{\frac{k_{\mathrm{B}} T_{\mathrm{kin}}}{\bar{m}}+\left(\frac{\Delta V^{2}}{8 \ln (2)}-\frac{k_{\mathrm{B}} T_{\mathrm{kin}}}{m}\right)}$,

where $k_{\mathrm{B}}$ is the Boltzmann constant, $\bar{m}=2.33 \mathrm{u}$ the mean molecular mass (total mass divided by the number of particles), and $m=29 \mathrm{u}$ the mass of the ${ }^{13} \mathrm{CO}$ molecule. We assume kinetic temperature is equal to dust temperature, a good assumption at the density of star-forming cores (Goldsmith 2001). Virial mass is then calculated as

$M_{\mathrm{vir}}=\frac{k R_{\mathrm{eff}} \sigma^{2}}{G}$,

where $k$ is a constant that depends on the density distribution. Assuming the density profile of the core scales with distance as $r^{-n}$ (MacLaren et al. 1988), $k$ can be calculated as

$k=\frac{5-2 n}{3-n}$.
As in Parikka et al. (2015), we assume $n=1.5$ and thus use a value of $k=1.333$. Virial mass ratio $\alpha_{\text {vir }}$ is calculated, with a critically bound core having observed mass equal to its virial mass. We define $\alpha_{\text {vir }}=M_{\text {vir }} / M_{\text {obs }}$, and require prestellar cores to have $\alpha_{\text {vir }} \leq 1$, as in Bresnahan et al. (2018).

\subsection{YSO association with clumps}

\subsubsection{Catalogs used}

We searched for potential YSOs from several catalogs. We used AKARI and Herschel point source catalogs, the YSO candidate catalog of Marton et al. (2016), and the Spitzer catalog of Gutermuth \& Heyer (2015). As coverage between catalogs varies, the fields covered by each catalog are listed in Table C.1.

The Akari/IRC point-source catalog (PSC) ${ }^{6}$ is based on the $9 \mu \mathrm{m}$ and $18 \mu \mathrm{m}$ observations of the AKARI satellite (Kataza et al. 2010) and the AKARI/FIS bright-source catalog (BSC) data from 65-160 $\mu \mathrm{m}$ observations (Yamamura et al. 2010). The Herschel PACS point source catalogs (hereafter PACS catalogs) include point sources at 70, 100, and $160 \mu \mathrm{m}$, as well as extended source and rejected source catalogs (Marton et al. 2017). For YSO detection, we used the 70 and $100 \mu \mathrm{m}$ catalogs. The YSO candidates in the Marton et al. (2016) catalog are based on source classification using the WISE (Wide-field IR Survey Explorer) satellite (Wright et al. 2010) data and the 2MASS (Two Micron All-Sky Survey) catalog (Skrutskie et al. 2006). The Marton catalog is divided into two sections: Class I and II YSO candidates, and Class III and more evolved YSO candidates. We used both of these. The Gutermuth catalog was created using the MIPSGAL $24 \mu \mathrm{m}$ Galactic Plane Survey matched with archival data from the 2MASS, GLIMPSE, and WISE surveys (Gutermuth \& Heyer 2015). We searched the catalog for those sources with spectra matching those of Class I and II YSOs. Sources from different catalogs were merged if their separations were smaller than $2^{\prime \prime}$.

The detection limit of the Herschel catalogs depends on the wider environment of the field, but also on the mass of the protostar. For YSOs with mass $1 M_{\odot}$, a circumstellar mass of $\geq 0.5 M_{\odot}$ is required for detection; YSOs of $10 M_{\odot}$ require circumstellar masses of only $\geq 0.0025 M_{\odot}$ (Gaczkowski et al. 2013). The AKARI catalogs are supposed to have uniform detection limits over the whole sky, of $\sim 0.7 \mathrm{Jy}$ for PSC, and $\sim 1.8 \mathrm{Jy}$ for the BSC (Kataza et al. 2010; Yamamura et al. 2010). In the different WISE bands a $S / N$ of 20 was achieved at $\sim 12$ mag (Cutri et al. 2012). In the 2 MASS source catalogs, a $10 \sigma$ detection level of $\geq 15$ was achieved over the entire sky (Skrutskie et al. 2006). The sensitivity of the MIPSGAL point sources is $\sim 2$ and $75 \mathrm{mJy}$ at 24 and $70 \mu \mathrm{m}$, respectively (Carey et al. 2009).

\subsubsection{Associating YSOs with clumps}

Not all YSOs in a field are physically associated with the detected clumps. The method for choosing clump association was based on spatial matching. If any of the clump's pixels are within the FWHM beam size of SCUBA-2 (14.6") of a YSO, then the YSO is considered potentially associated with that clump and the clump is characterized tentatively as protostellar. If a YSO is near the boundary of two or more clumps, it is associated with the clump with most pixels within an 14.6" distance of it. Otherwise, the YSO is considered a field YSO.

\footnotetext{
$\overline{6}$ https://skyview.gsfc .nasa.gov/current/cgi/query.pl
} 


\subsubsection{Confirming YSO candidates}

To seek further corroboration of the physical connection between YSO candidates and clumps, we compare the mean intensity of a circle within $12^{\prime \prime}$ of the YSO in PACS $160 \mu \mathrm{m}$ with that in SPIRE $250 \mu \mathrm{m}$. Before this analysis the PACS images are converted to $\mathrm{MJy} \mathrm{sr}^{-1}$ and both fields are convolved to $20^{\prime \prime}$ resolution. The average signal in a reference region chosen from a low-intensity region of each map is used to subtract the background. Furthermore, other YSO candidates in the field are masked to prevent contamination. The increased temperature at the YSO location results in increased short-wavelength emission, which should be visible as higher intensity at $160 \mu \mathrm{m}$. This is compared to a reference annulus between $36^{\prime \prime}$ and $84^{\prime \prime}$ of the YSO. We define the change in Herschel intensity ratios $\Delta C$ as the difference between the ON and OFF areas. To be significant, $\Delta C$ is required to be significantly positive compared to the fluctuations, that is,

$\Delta C=\left(\frac{I_{160}}{I_{250}}\right)_{\mathrm{ON}}-\left(\frac{I_{160}}{I_{250}}\right)_{\mathrm{OFF}}>\xi \times \sigma\left(\frac{I_{160}}{I_{250}}\right)$,

where $\xi$ is a threshold value that takes into account the convolution of the original data and $I_{160}$ and $I_{250}$ are the $160 \mu \mathrm{m}$ and $250 \mu \mathrm{m}$ intensities, respectively. The quantity $\sigma$ refers to the standard deviation of the ratios. We estimate $\xi$ by simulating maps of white noise and taking the $95 \%$ probability value of the distribution of $\Delta C$. We find a value $\xi=12.98$.

In figures and tables, we refer to clumps that have additional confirmation from a significantly positive $\Delta C$ as PS-C, and those that do not as PS. We note that Herschel intensity ratio does not provide absolute confirmation of the YSO, only a stronger probability of association with the clump in question.

\section{Results}

In this section we present the results of our analysis of these data, which are discussed in more detail in Sect. 5. The fields show characteristics we would expect from dense regions. In this paper, all sources are called clumps even though they span a large range of linear sizes, from nearby cores to entire distant clouds. Owing to the resolution of the SCUBA-2 instrument, individual cores are not resolved in distant fields.

\subsection{Distances of the examined fields}

Distances to 23 fields were estimated in Montillaud et al. (2015), where the authors use kinematic distance and extinction modeling. Distances to ten fields have been estimated in the PGCC (Planck Collaboration XXVIII 2016); four of these also in Montillaud et al. (2015). The distance estimates in the two catalogs are consistent within their uncertainties.

Twenty-seven new kinematic distances were derived from line-of-sight velocities of ${ }^{12} \mathrm{CO}$ spectra from TRAO (see Sect. 3.1). The derived galacto- and heliocentric kinematic distances are listed in Table D.1, with all distances used in this paper in Table D.2. Assuming uncertainties of $\sim 5 \mathrm{~km} \mathrm{~s}^{-1}$ in velocity dispersion (Stark 1984; Clemens 1985; Whitaker et al. 2017), we derive a final uncertainty in $v_{\text {LSR }}$ of $50 \%$. Adopting uncertainties of $0.2 \mathrm{kpc}$ for $R_{0}, 8 \mathrm{~km} \mathrm{~s}^{-1}$ for $v_{0}$ (Reid et al. 2014), and $5^{\prime}$ for $l$ and $b$ (the resolution of Planck), we derive an uncertainty of $\sim 51 \%$ for galacto- and heliocentric distances. As we are unable to solve the near-far kinematic distance ambiguity, we increase the uncertainty in heliocentric distance to ${ }_{-51}^{+100} \%$. Montillaud et al. (2015) provide uncertainty estimates for their derived distances, which are on average $53 \%$, and we adopt an uncertainty of $30 \%$ for the distances from the PGCC that do not have previous uncertainty estimates. The fields in this sample are located at distances of $0.1-4.5 \mathrm{kpc}$, with a mean at around $1 \mathrm{kpc}$. Near $\ell \sim 0^{\circ}$, many fields are likely to be associated with the Sagittarius arm and near $\ell \sim 180^{\circ}$ with the Perseus arm (see Fig. 3 in Beuther et al. 2012). We also note that while we analyzed the projected two-dimensional distance between clumps, it is possible that these clumps are more distant along the line of sight. To solve this question, molecular line data of all of the clumps would be required.

\subsection{The general ISM}

We calculated the intensity, dust temperature, column density, and optical depth for the 45 Herschel fields with full SPIRE and PACS $160 \mu \mathrm{m}$ data. Analysis was performed with constant $\beta=1.8$ as well as varying $\beta$. The PACS and SPIRE fields were background subtracted. The coordinates of the reference regions used for zero-point correction are listed in Appendix F. The same analysis was performed using only the SPIRE 250$500 \mu \mathrm{m}$ fields relying on the zero points set in the archival maps. Derived temperature, column density, and spectral index maps are in Appendix $\mathrm{H}$ and the average values are listed in Table 2. We plot histograms of temperature and opacity spectral index for all fields with SPIRE and PACS data in Fig. 3. As PACS and SPIRE coverage varies within individual fields, this figure includes only pixels that have data at all wavelengths.

The average color temperature of the fields is $18.8 \mathrm{~K}$ and the average column density $N\left(\mathrm{H}_{2}\right)=0.4 \times 10^{21} \mathrm{~cm}^{-2}$. Using only $250-500 \mu \mathrm{m}$ data results in lower temperature, higher optical depth, and higher column density estimates. This is expected as $160 \mu \mathrm{m}$ emission is more sensitive to warm dust along the line of sight. The temperature derived from $160-500 \mu \mathrm{m}$ data ranges from $7 \mathrm{~K}$ to $40 \mathrm{~K}$, and column densities from $2.7 \times 10^{19}$ to $1.5 \times 10^{22} \mathrm{~cm}^{-2}$. We plot temperature and column density as a function of Galactic latitude $b$ and Galactic longitude $\ell$ in Fig. 4. Both mean temperature and column density are highest at low Galactic latitudes, although the high-latitude bins $\left(|b|>10^{\circ}\right)$ often contain only one or two nearby fields.

The mean spectral index of the fields is around 1.7-1.9; the standard deviation under 0.5 . $\beta$ ranges from 0.6 to 3.5. Variations of opacity spectral index do not, in general, have a large effect on the properties derived for the regions. Only the changes in optical depth and column density (probabilities $p=0.09$ and $p=0.001$ for SPIRE and SPIRE+PACS, respectively for the null hypothesis of no change) are significant (see Table 3 ). Thus we use maps with constant spectral index for analysis of dense clumps.

\subsection{The temperature- $\beta$ relation}

The temperature dependence of opacity spectral index $\beta$ is a much-studied feature, which provides information on the chemical composition, structure, and size distribution of interstellar dust grains (Ossenkopf \& Henning 1994); (Kruegel \& Siebenmorgen 1994; Mennella et al. 1998; Boudet et al. 2005; Meny et al. 2007; Compiègne et al. 2011; Juvela et al. 2011). However, as instrumental noise causes false anticorrelation between temperature and dust opacity spectral index (Shetty et al. 2009a,b; Juvela \& Ysard 2012; Juvela et al. 2013), how much of this anticorrelation is physical is uncertain.

We examined the $(T, \beta)$ values from the $160-500 \mu \mathrm{m}$ and $250-500 \mu \mathrm{m}$ fits with the function 
Table 2. Parameters of the full Herschel fields.

\begin{tabular}{lccccc}
\hline \hline $\begin{array}{l}\text { Wavelength } \\
\text { used }\end{array}$ & $\begin{array}{c}\langle T\rangle \\
(\mathrm{K})\end{array}$ & $\begin{array}{c}\langle I\rangle \\
\left(\mathrm{MJy} \mathrm{sr}^{-1}\right)\end{array}$ & $\begin{array}{c}\left\langle N\left(\mathrm{H}_{2}\right)\right\rangle \\
\left(10^{21} \mathrm{~cm}^{-2}\right)\end{array}$ & $\begin{array}{c}\langle\tau\rangle \\
\left(10^{-3}\right)\end{array}$ & $\langle\beta\rangle$ \\
\hline $250-500 \mu \mathrm{m}$ & $17.1 \pm 3.1$ & $261.2 \pm 480.9$ & $2.6 \pm 2.9$ & $1.7 \pm 1.9$ & 1.8 \\
$160-500 \mu \mathrm{m}$ & $20.3 \pm 6.7$ & $142.5 \pm 338.8$ & $1.3 \pm 2.2$ & $0.9 \pm 1.5$ & 1.8 \\
\hline $250-500 \mu \mathrm{m}$ & $16.5 \pm 3.5$ & $261.0 \pm 481.1$ & $3.1 \pm 3.2$ & $2.1 \pm 2.1$ & $1.9 \pm 0.3$ \\
$160-500 \mu \mathrm{m}$ & $22.7 \pm 8.5$ & $142.2 \pm 338.2$ & $1.2 \pm 2.2$ & $0.8 \pm 1.5$ & $1.7 \pm 0.7$ \\
\hline Average: & $17.7 \pm 4.8$ & $239.7 \pm 460.9$ & $2.6 \pm 3.0$ & $1.7 \pm 2.0$ & $1.9 \pm 0.4$ \\
\hline
\end{tabular}

Notes. Mean temperature $T$, intensity $I_{250}$, column density $N\left(\mathrm{H}_{2}\right)$, optical depth $\tau_{250}$, and opacity spectral index $\beta$ calculated for the full map, for pixels with value over 0 , using the various methods. The first two maps have constant spectral index.
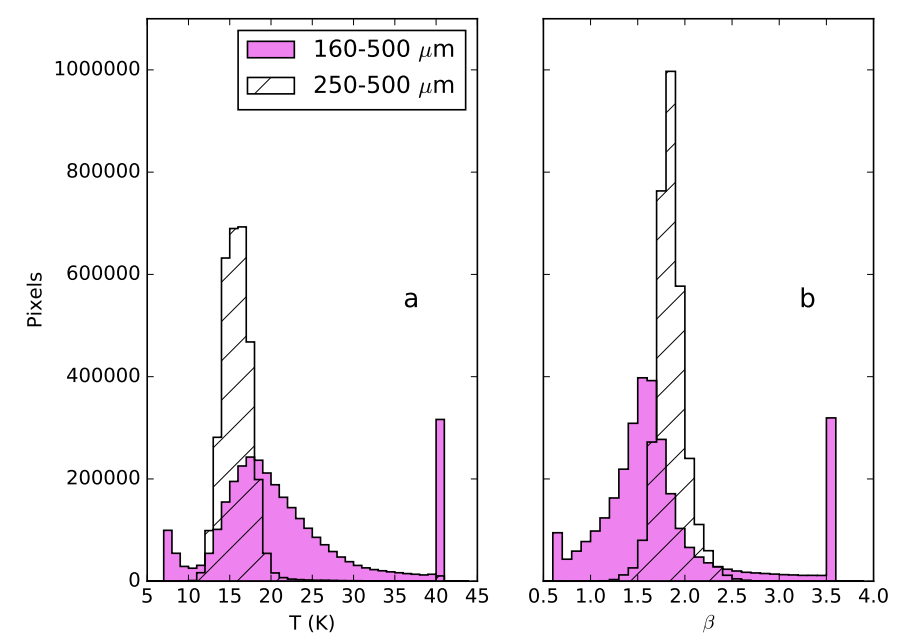

Fig. 3. Temperature $(a)$ and spectral index $(b)$ histograms for the entire fields, computed with SED fits of the $250-500 \mu \mathrm{m}$ (hatched histogram) and $160-500 \mu \mathrm{m}$ (purple histograms) data. Only areas covered by both PACS and SPIRE observations are used. As the MBB script fits temperatures between $5-40 \mathrm{~K}$, and spectral index between 0.6 and 3.5 , thus the first and last bins include all pixels with $T$ or $\beta$ outside of these ranges.

$\beta(T ; A, \alpha)=A \times T^{-\alpha}$

(Désert et al. 2008). Fitting data in the range $T=8-39 \mathrm{~K}$ and $\beta=0.6-3.5$ resulted in $\beta=7.01 \times T^{-0.47}$ (Table 4 , Fig. 5). We also examined the relation separately at the YSO and clump locations using 160-500 $\mu \mathrm{m}$ data (Fig. 6). The relation appears to be steeper at the location of YSOs compared to at dense clumps or over the entire fields. However at the location of YSOs we have only 113 pixels, compared to 15920 at clumps. To test whether the differing numbers of points had an effect on the derived slope of the relation, we randomly selected 113 pixels from the clump locations and calculated the temperature- $\beta$ relation, repeating this $137(=15920 / 133)$ times. We find a mean slope of $\langle\alpha\rangle=0.3 \pm 0.1$, showing that the temperature- $\beta$ relation is steeper at the location of YSOs. To estimate the accuracy of all these derived relations, we compare our results with simulations where the true spectral index values are independent of temperature.

To estimate the effects of this contamination by noise, we performed Monte Carlo simulations using 1000 data points with the mean and standard deviation after addition of noise matching that of our data. We fixed the value of $\beta$ to 1.8 and calculate intensities from Eq. (1). To this intensity we added Gaussian noise at $2 \%$ of intensity for SPIRE wavelengths and $5 \%$ at $160 \mu \mathrm{m}$, the predicted uncertainties for the two instruments (Valtchanov 2017). We calculated the new temperature and spectral index for
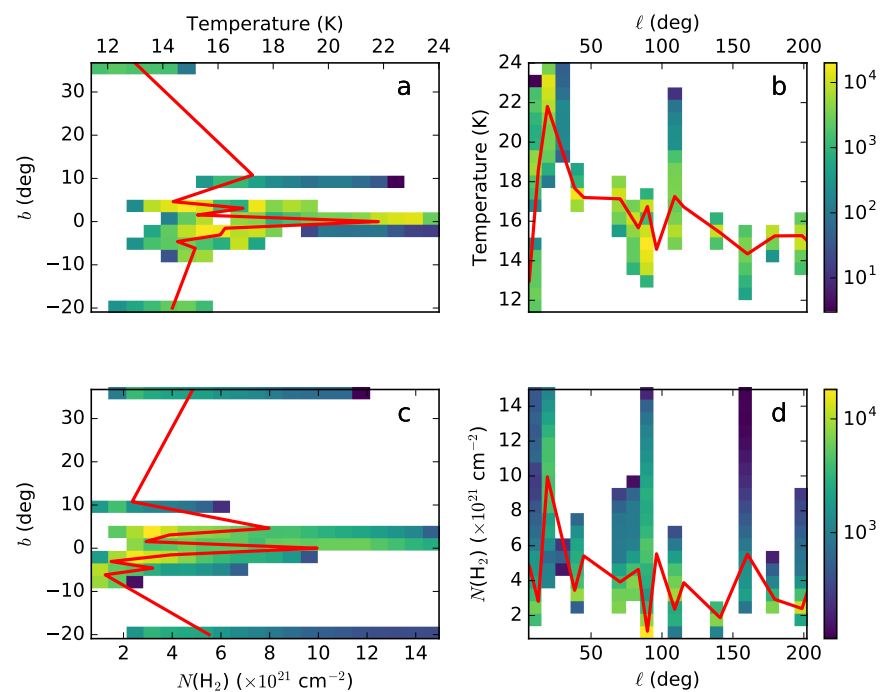

Fig. 4. Average properties of the 53 fields. The frames show 2D histograms of (a) temperature plotted against Galactic latitude $b$, (b) temperature plotted against Galactic longitude $\ell,(c)$ column density plotted against Galactic latitude $b$, and $(d)$ column density plotted against Galactic longitude $\ell$. The red line plots the averages for each bin. The plots are based on fits to SPIRE data only.

Table 3. Effect of constant vs. variable spectral index on derived field properties.

\begin{tabular}{|c|c|c|c|c|c|}
\hline \multicolumn{3}{|c|}{ SPIRE maps only } & \multicolumn{3}{|c|}{ SPIRE and PACS maps } \\
\hline Variable & $t$ & $p$ & Variable & $t$ & $p$ \\
\hline$T$ & 0.69 & 0.497 & $T$ & -0.13 & 0.895 \\
\hline$I$ & $<10^{-2}$ & 0.998 & $I$ & $<10^{-2}$ & 0.999 \\
\hline$N\left(\mathrm{H}_{2}\right)$ & -1.73 & 0.091 & $N\left(\mathrm{H}_{2}\right)$ & -3.57 & 0.001 \\
\hline$\tau$ & -1.73 & 0.091 & $\tau$ & -3.57 & 0.001 \\
\hline
\end{tabular}

Notes. Student- $t$ test comparing the mean values of maps with constant and variable spectral index, for SPIRE maps only (Cols. 1-3) and SPIRE+PACS $160 \mu \mathrm{m}$ maps (Cols. 4-6).

this noisy data using the methodology in Sect. 3.2, and we calculated the $A$ and $\alpha$ parameters, which are compared to those of our derived relation. We repeated this 1000 times. The histogram of derived $\alpha$ is plotted in gray in Fig. 7. We find $100 \%$ of derived values of $\alpha$ to be below 0.47 , which suggests that some of the $T-\beta$ anticorrelation is not due to noise. We also perform an additional test, by doubling noise to $4 \%$ of intensity at $250-500 \mu \mathrm{m}$ and $10 \%$ at $160 \mu \mathrm{m}$, and plot this in orange in Fig. 7. With this, we find $99.9 \%$ of values above 4.7 . 
Table 4. Parameters of the fitted $\beta=A \times T^{-\alpha}$ relations.

\begin{tabular}{lcc}
\hline \hline Dataset & A & $\alpha$ \\
\hline $250-500 \mu \mathrm{m}$ & 6.32 & 0.43 \\
$160-500 \mu \mathrm{m}$ & 9.30 & 0.57 \\
Both datasets & 7.01 & 0.47 \\
At YSOs & 13.7 & 0.72 \\
At clumps & 5.76 & 0.46 \\
\hline
\end{tabular}

Notes. Coefficients derived for Eq. (12) using $\chi^{2}$ method. The last two rows use 160-500 $\mu \mathrm{m}$ data, taking into account only those pixels at YSOs or clumps, and for which both temperature and spectral index are above zero.
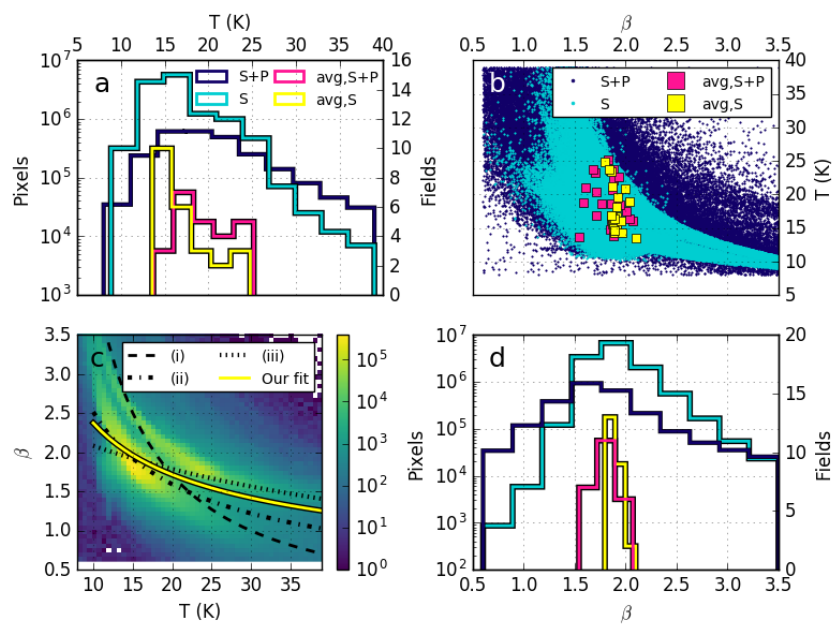

Fig. 5. (a) Histogram of field temperature. (b) Spectral index plotted against temperature. (c) 2D histogram of temperature plotted against spectral index. $(d)$ Histogram of field spectral index. The values are calculated from background-subtracted data in the ranges $250-500 \mu \mathrm{m}$ (light blue, S) and 160-500 $\mu \mathrm{m}$ (dark blue, S+P). In frames $a$ and $d$, these histograms correspond to the left-hand $y$-axis. Every tenth value of those pixels with $9<T<39 \mathrm{~K}$ and $0.6<\beta<3.4$ is included in frame $b$ and every pixel in the other frames. Yellow and pink stand for averages of each field $(n=24)$ with the four-map $(\operatorname{avg}, \mathrm{S}+\mathrm{P})$ and three-map (avg, S) combinations, respectively, and in frames $a$ and $d$ correspond to the right-hand $y$-axis. $\beta$ - $T$ relations from the literature are also plotted in (c), where (i) $\beta=1.71 \times(T / 20 \mathrm{~K})^{-1.33}$ (Paradis et al. 2010), dashed line, (ii) $\beta=11.5 \times T^{-0.66}$ (Désert et al. 2008), dashdotted line, and (iii) $\beta=1 /(0.4+0.008 \times T)$ (Dupac et al. 2003), dotted line. The relation derived for our data is plotted on frame $c$ in yellow.

\subsection{Herschel spectra and $850 \mu \mathrm{m}$ measurements}

Because of previous reports of spectral index variations as a function of frequency (Reach et al. 1995; Paradis et al. 2012; Juvela et al. 2015; Mason et al. 2020), we wished to extend the SED analysis to SCUBA-2 data at $850 \mu \mathrm{m}$. In the comparison with satellite data, a major concern is the spatial filtering that the sky-noise reduction introduces into the SCUBA-2 maps. This could be taken into account by filtering the Herschel observations so that the loss of low spatial frequencies would be similar to the filtering in the ground-based observations (Sadavoy et al. 2013; Juvela et al. 2018b). However, we concentrate here only on the cores at small spatial scales where the effects of filtering are smaller. Sadavoy et al. (2013) simulated flux loss due to SCUBA-2 filtering, finding that on scales below $2.5^{\prime}$ objects are fully recovered. We also examined flux loss using Herschel $250 \mu \mathrm{m}$ maps, comparing the clump fluxes extracted from the
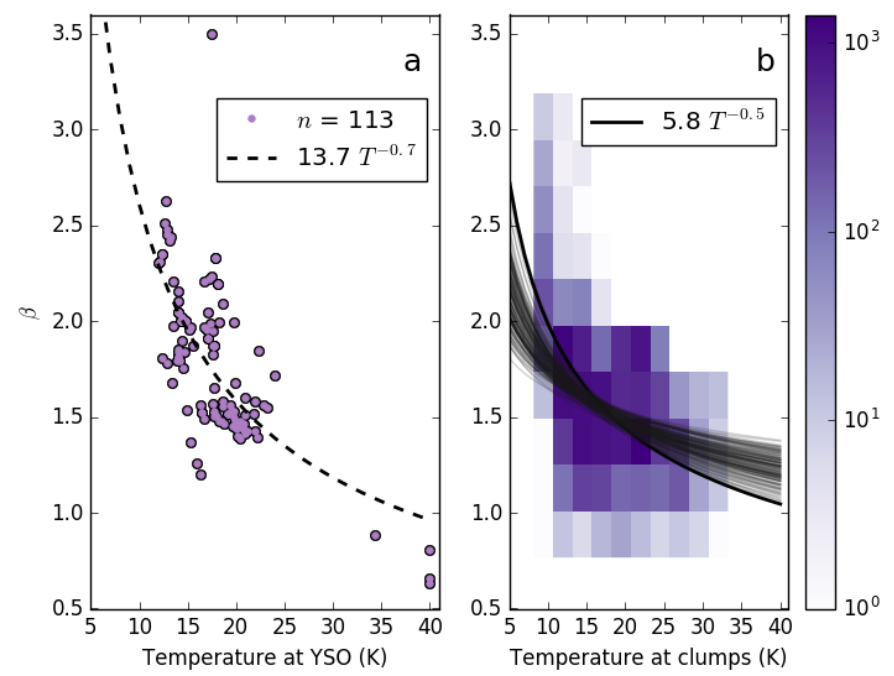

Fig. 6. Temperature- $\beta$ relation, taking into consideration only those pixels where $(a)$ a YSO is located ( $n=113$ pixels) and $(b)$ at the location of clumps ( $n=15920$ pixels). The color bar corresponds to the number of pixels in the case of $b$. We plot the calculated relations for 113 random points from the clumps sample in gray in frame $b$.

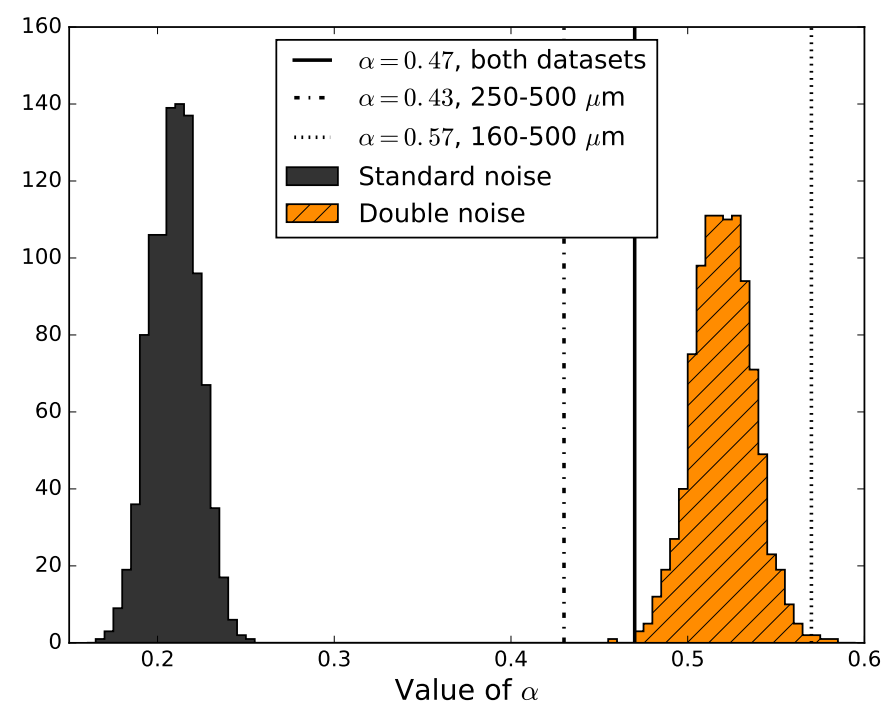

Fig. 7. Histogram of derived values of $\alpha$ using 1000 sets of 1000 SEDs each, with assumed observational noise (black) and double assumed noise (orange). Our derived relations are plotted with a black dotted line.

original maps with maps run through the SCUBA-2 reduction pipeline. The approach is therefore the same as in Sadavoy et al. (2013), but in this work we use the actual structures in the maps and similar values for the aperture and reference-annulus size as in the SCUBA-2 analysis. In the sample of 15 clumps, the filtering caused a flux loss of $(1.3 \pm 6.2) \%$, where the flux loss is under $5 \%$ in $80 \%$ of cases. The filtering thus introduces some scatter but only a small bias, which is statistically compatible with zero.

We convolved the $250-850 \mu \mathrm{m}$ maps to a common $40^{\prime \prime}$ resolution and color corrected them assuming a MBB spectrum $B_{v}(T=17 K) v^{1.8}$. We constructed SEDs from clump peak values from which the local background was subtracted. The background estimates correspond to the median over an ellipse that starts at a distance of 0.7 times the clump FWHM (using the 

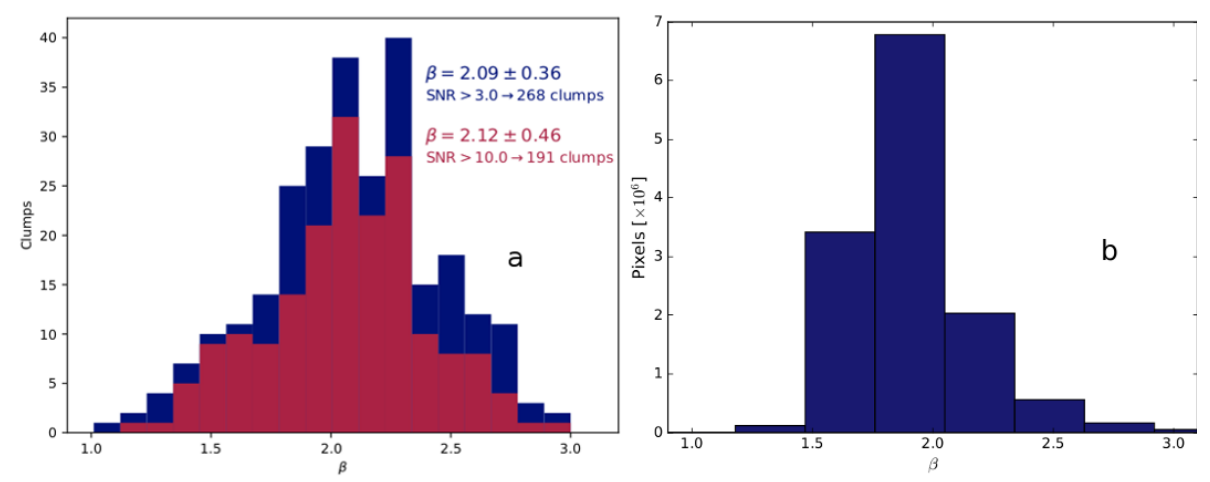

Fig. 8. (a) Values of opacity spectral index for clumps with $S / N>3$ (blue) and $S / N>6$ (red). (b) Values of derived opacity spectral index over all pixels over the fields in in our sample. major and minor axis FWHM values) and is $30^{\prime \prime}$ wide. The SCUBA-2 maps should recover spatial scales up to $\sim 200^{\prime \prime}$ and because the median FWHM size of the clumps is less than $1^{\prime}$, the systematic effects from the spatial filtering should remain small compared to other sources of uncertainty. We do not apply any aperture corrections because we are only interested in the SED shape and all input data are already at the same resolution and even use the same pixelization.

We selected the most reliable set of cores by setting a threshold for the minimum signal-to-noise ratio $(\mathrm{S} / \mathrm{N})$ in each band. The 250-500 $\mu \mathrm{m}$ data were fitted with MBBs, estimating the uncertainties with a Markov chain Monte Carlo procedure. Figure I. 1 shows the fits for nine random clumps with $S / N>3$ in all bands. The green boxes indicate the 5\%-95\% confidence interval for the extrapolated $850 \mu \mathrm{m}$ value. In most cases the observed $850 \mu \mathrm{m}$ point falls inside the box.

The distributions of spectral index values (Fig. 8a) are consistent with those derived in Sect. 4.2 (Fig. 8b), although here the values represent the background-subtracted emission of the clump centers only. Figure 9 shows the distributions of the residuals when the SED prediction is subtracted from the $850 \mu \mathrm{m}$ observation. These peak close to zero. Moreover, the plot also includes the predicted distribution for the $S / N>6$ cores, which is calculated assuming a zero mean and for individual clumps the error distributions predicted by the uncertainties of the $850 \mu \mathrm{m}$ observed and SED-predicted values. The sample shows neither indications of a systematic spectral index variation beyond $500 \mu \mathrm{m}$ nor any source-to-source variations beyond the observational uncertainties. We detect no correlation between column density and excess. Our detected $850 \mu \mathrm{m}$ excess is consistent with zero, although a marginal excess may exist when SCUBA-2 flux loss is taken into account. In Appendix B we further compare the temperatures, spectral index values, and masses derived for the clumps using different combinations of the $160-850 \mu \mathrm{m}$ bands. We find that the inclusion of the $160 \mu \mathrm{m}$ point systematically decreases the temperature estimates, while the inclusion of the $850 \mu \mathrm{m}$ point slightly increases the spectral index estimates.

\subsection{Detected clumps}

FellWalker analysis found a total of 529 clumps in the 53 fields. Each field contains between 1 and 35 clumps, with an average of 10 clumps per field, although in general fields closer to the plane of the Galaxy also contain more clumps. The clumps are generally found on filamentary structures. The clumps found in each field are plotted on top of SCUBA-2 intensity maps in Appendix J.

Clumps are cold and dense, with temperatures around 10$25 \mathrm{~K}$ and average column density on the order of $10^{22} \mathrm{~cm}^{-2}$.

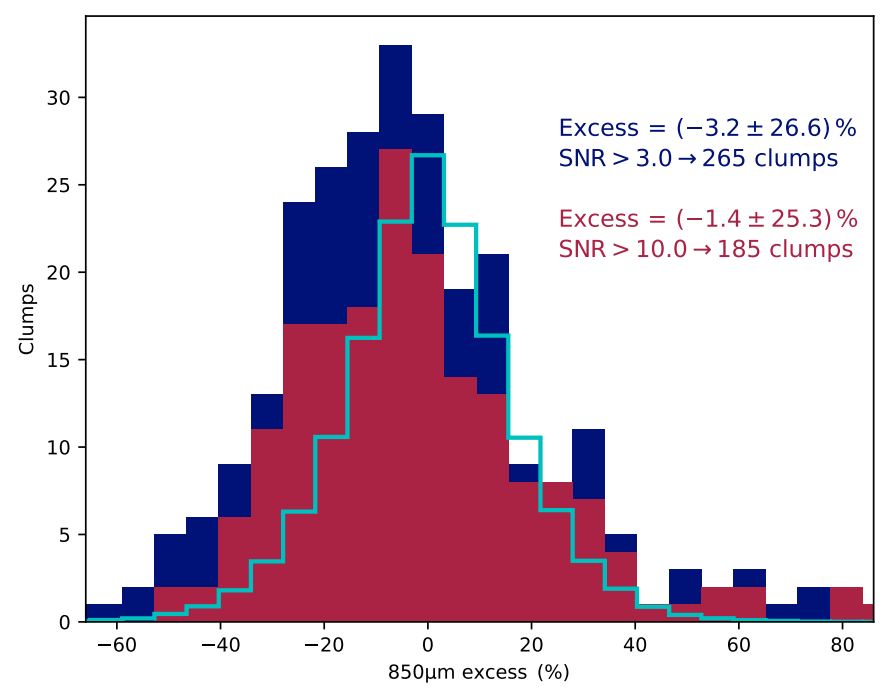

Fig. 9. SCUBA-2 $850 \mu \mathrm{m}$ excess fit for clumps with $S / N>3$ (blue) and $S / N>6$ (red). The turquoise line is the predicted distribution for the $S / N>6$ cores that is calculated assuming a zero mean.

Clump masses range from $10^{-2}$ to $10^{3} M_{\odot}$, with an average mass on the order of $10^{2} M_{\odot}$. The flux density is approximately $2 \mathrm{Jy}$. Properties are estimated as described in Sect. 3.4, and are discussed further in Sect. 4.8. We present a catalog of derived clump properties in Appendix K.

\subsection{YSO detections}

The YSOs that are not associated with any clump are referred to as field YSOs. Clumps that are spatially associated with at least one YSO are tentatively classified as protostellar. The possibilities of misclassifying clumps as protostellar are discussed further in Sect. 4.7. We also plot clump-associated YSOs on top of the clumps in Appendix J. The mean distance to field YSOs is slightly higher: $2 \mathrm{kpc}$ compared to $1.8 \mathrm{kpc}$ for clump-associated YSOs. This is to be expected, as nearby clumps are generally high-latitude clumps with fewer field YSOs. Over half of the YSO candidates located in the fields are not associated with any clump.

\subsection{Virial analysis and clump classification}

We use virial analysis to predict whether the self-gravity of the clump is strong enough to prevent dissipation due to internal turbulence (Sect. 3.5). We use ${ }^{13} \mathrm{CO}$ line data from Wang et al. (2018), which covers 13 clumps in three fields: G105.44+09.88, 
Table 5. Estimated mean parameters for different clump categories.

\begin{tabular}{|c|c|c|c|c|c|c|c|c|c|c|c|}
\hline $\begin{array}{l}\text { (1) } \\
\text { Clump } \\
\text { type }\end{array}$ & $\begin{array}{l}(2) \\
\langle T\rangle \\
(\mathrm{K}) \\
\end{array}$ & $\begin{array}{c}(3) \\
\left\langle F_{v}(850 \mu \mathrm{m})\right\rangle \\
(\mathrm{Jy})\end{array}$ & $\begin{array}{c}(4) \\
\left\langle N\left(\mathrm{H}_{2}\right)\right\rangle \\
\left(10^{21} \mathrm{~cm}^{-2}\right) \\
\end{array}$ & $\begin{array}{c}(5) \\
\left\langle N\left(\mathrm{H}_{2}\right)_{850}\right\rangle \\
\left(10^{21} \mathrm{~cm}^{-2}\right) \\
\end{array}$ & $\begin{array}{c}(6) \\
\text { Ratio } \\
\text { (peak/mean) }\end{array}$ & $\begin{array}{l}(7) \\
\langle\beta\rangle\end{array}$ & $\begin{array}{c}(8) \\
\langle M\rangle \\
\left(M_{\odot}\right)\end{array}$ & $\begin{array}{c}(9) \\
\left\langle M_{\mathrm{vir}}\right\rangle \\
\left(M_{\odot}\right)\end{array}$ & $\begin{array}{c}(10) \\
\langle d\rangle \\
(\mathrm{kpc})\end{array}$ & \multicolumn{2}{|c|}{$N_{\text {clumps }}$} \\
\hline \multicolumn{12}{|c|}{ All fields with distance, $n=395$ clumps } \\
\hline All & $17.8 \pm 3.6$ & $2.4 \pm 3.9$ & $14.5 \pm 6.7$ & $30.0 \pm 39.1$ & $2.2 \pm 4.5$ & $1.7 \pm 0.1$ & $135.7 \pm 316.0$ & $9.8 \pm 7.4$ & $2.4 \pm 1.3$ & 264 & 395 \\
\hline PS-C & $16.5 \pm 3.3$ & $2.8 \pm 2.7$ & $17.8 \pm 8.1$ & $52.9 \pm 52.2$ & $2.1 \pm 5.7$ & $1.7 \pm 0.1$ & $176.8 \pm 300.6$ & $9.4 \pm 7.1$ & $2.3 \pm 1.6$ & 36 & 36 \\
\hline PS & $17.7 \pm 3.3$ & $4.2 \pm 5.8$ & $15.7 \pm 7.8$ & $43.1 \pm 56.5$ & $2.5 \pm 2.4$ & $1.8 \pm 0.1$ & $233.4 \pm 552.5$ & $11.0 \pm 8.1$ & $2.2 \pm 1.2$ & 52 & 90 \\
\hline PRE & $18.2 \pm 3.6$ & $2.0 \pm 3.0$ & $14.1 \pm 5.7$ & $23.7 \pm 26.0$ & $2.8 \pm 2.1$ & $1.7 \pm 0.1$ & $107.0 \pm 171.1$ & $10.2 \pm 7.0$ & $2.6 \pm 1.2$ & 154 & 245 \\
\hline SL & $17.3 \pm 4.4$ & $0.4 \pm 0.2$ & $9.9 \pm 3.7$ & $11.0 \pm 5.5$ & $1.2 \pm 0.6$ & $1.8 \pm 0.1$ & $1.5 \pm 1.0$ & $2.3 \pm 1.2$ & $0.7 \pm 0.2$ & 22 & 24 \\
\hline SL PRE & $18.1 \pm 3.7$ & $1.8 \pm 2.9$ & $13.6 \pm 5.6$ & $22.5 \pm 25.1$ & $2.2 \pm 1.9$ & $1.8 \pm 0.1$ & $97.6 \pm 166.0$ & $9.5 \pm 7.1$ & $2.4 \pm 1.2$ & 176 & 269 \\
\hline PS PS-C & $17.2 \pm 3.4$ & $3.8 \pm 5.1$ & $16.5 \pm 8.0$ & $45.9 \pm 55.5$ & $2.2 \pm 5.0$ & $1.7 \pm 0.1$ & $217.2 \pm 494.5$ & $10.6 \pm 7.9$ & $2.2 \pm 1.3$ & 88 & 126 \\
\hline \multicolumn{12}{|c|}{$d<0.5 \mathrm{kpc}, n=13$ clumps } \\
\hline All & $11.9 \pm 1.0$ & $1.1 \pm 1.0$ & $14.1 \pm 5.8$ & $20.9 \pm 11.2$ & $2.0 \pm 1.0$ & $1.8 \pm 0.1$ & $1.7 \pm 1.8$ & $1.4 \pm 0.7$ & $0.4 \pm 0.1$ & 8 & 13 \\
\hline PS & - & $1.0 \pm 0.6$ & - & $15.3 \pm 5.5$ & - & - & $1.4 \pm 0.8$ & $1.6 \pm 0.6$ & $0.4 \pm 0.0$ & 0 & 3 \\
\hline PRE & $12.1 \pm 1.2$ & $2.1 \pm 1.0$ & $15.2 \pm 5.2$ & $34.9 \pm 9.1$ & $2.5 \pm 0.9$ & $1.8 \pm 0.1$ & $3.5 \pm 2.1$ & $1.9 \pm 0.8$ & $0.4 \pm 0.1$ & 4 & 4 \\
\hline SL & $11.7 \pm 0.7$ & $0.5 \pm 0.1$ & $13.1 \pm 6.1$ & $14.3 \pm 3.5$ & $1.5 \pm 0.9$ & $1.8 \pm 0.1$ & $0.7 \pm 0.5$ & $1.0 \pm 0.5$ & $0.3 \pm 0.2$ & 4 & 6 \\
\hline SL PRE & $11.9 \pm 1.0$ & $1.1 \pm 1.0$ & $14.1 \pm 5.8$ & $22.6 \pm 11.9$ & $1.5 \pm 0.9$ & $1.8 \pm 0.1$ & $1.8 \pm 2.0$ & $1.4 \pm 0.8$ & $0.3 \pm 0.2$ & 8 & 10 \\
\hline \multicolumn{12}{|c|}{$0.5 \leq d<1.5 \mathrm{kpc}, n=99$ clumps } \\
\hline All & $15.8 \pm 3.5$ & $1.9 \pm 2.7$ & $13.8 \pm 6.6$ & $34.6 \pm 45.9$ & $2.3 \pm 2.1$ & $1.7 \pm 0.1$ & $13.7 \pm 18.8$ & $3.4 \pm 1.5$ & $0.8 \pm 0.1$ & 99 & 99 \\
\hline PS-C & $14.0 \pm 1.4$ & $2.7 \pm 2.7$ & $16.6 \pm 8.1$ & $66.0 \pm 62.4$ & $2.2 \pm 2.4$ & $1.7 \pm 0.1$ & $23.0 \pm 24.4$ & $3.6 \pm 1.5$ & $0.8 \pm 0.1$ & 19 & 19 \\
\hline PS & $17.0 \pm 3.8$ & $3.5 \pm 3.8$ & $15.0 \pm 8.6$ & $40.2 \pm 51.5$ & $2.4 \pm 1.5$ & $1.8 \pm 0.1$ & $20.7 \pm 24.7$ & $3.9 \pm 1.9$ & $0.8 \pm 0.1$ & 21 & 21 \\
\hline PRE & $14.7 \pm 2.7$ & $1.4 \pm 1.9$ & $13.9 \pm 4.4$ & $28.2 \pm 33.6$ & $3.6 \pm 2.3$ & $1.7 \pm 0.1$ & $11.0 \pm 11.5$ & $3.5 \pm 1.4$ & $0.9 \pm 0.1$ & 41 & 41 \\
\hline SL & $18.6 \pm 3.8$ & $0.4 \pm 0.3$ & $9.2 \pm 2.4$ & $9.9 \pm 5.6$ & $1.1 \pm 0.5$ & $1.8 \pm 0.1$ & $1.8 \pm 0.9$ & $2.7 \pm 1.1$ & $0.8 \pm 0.1$ & 18 & 18 \\
\hline SL PRE & $15.9 \pm 3.6$ & $1.1 \pm 1.7$ & $12.5 \pm 4.5$ & $22.6 \pm 29.4$ & $2.4 \pm 2.1$ & $1.7 \pm 0.1$ & $8.2 \pm 10.5$ & $3.2 \pm 1.4$ & $0.9 \pm 0.1$ & 59 & 59 \\
\hline PS PS-C & $15.6 \pm 3.3$ & $3.1 \pm 3.4$ & $15.8 \pm 8.4$ & $52.5 \pm 58.4$ & $2.3 \pm 2.1$ & $1.7 \pm 0.1$ & $21.8 \pm 24.6$ & $3.8 \pm 1.7$ & $0.8 \pm 0.1$ & 40 & 40 \\
\hline \multicolumn{12}{|c|}{$1.5 \leq d<3.0 \mathrm{kpc}, n=168$ clumps } \\
\hline All & $18.5 \pm 2.5$ & $2.9 \pm 4.8$ & $14.8 \pm 6.4$ & $30.5 \pm 39.3$ & $2.7 \pm 8.7$ & $1.7 \pm 0.1$ & $114.3 \pm 203.1$ & $9.5 \pm 4.5$ & $2.3 \pm 0.2$ & 63 & 168 \\
\hline PS-C & $15.7 \pm 0.5$ & $1.2 \pm 0.6$ & $8.9 \pm 1.3$ & $23.1 \pm 12.0$ & $2.7 \pm 10.1$ & $1.6 \pm 0.2$ & $63.3 \pm 42.7$ & $9.5 \pm 1.5$ & $2.2 \pm 0.2$ & 3 & 3 \\
\hline PS & $18.2 \pm 3.1$ & $4.4 \pm 6.4$ & $13.9 \pm 6.7$ & $43.8 \pm 55.4$ & $2.6 \pm 3.3$ & $1.7 \pm 0.1$ & $171.8 \pm 274.5$ & $11.0 \pm 4.7$ & $2.2 \pm 0.2$ & 15 & 47 \\
\hline PRE & $18.8 \pm 2.2$ & $2.3 \pm 3.8$ & $15.5 \pm 6.3$ & $25.4 \pm 29.5$ & $2.8 \pm 1.7$ & $1.7 \pm 0.1$ & $92.6 \pm 163.7$ & $8.9 \pm 4.4$ & $2.3 \pm 0.2$ & 45 & 118 \\
\hline PS PS-C & $17.8 \pm 3.0$ & $4.3 \pm 6.3$ & $13.1 \pm 6.4$ & $42.6 \pm 54.1$ & $2.7 \pm 8.9$ & $1.7 \pm 0.1$ & $165.3 \pm 267.5$ & $10.9 \pm 4.6$ & $2.2 \pm 0.2$ & 18 & 50 \\
\hline \multicolumn{12}{|c|}{$d \geq 3.0 \mathrm{kpc}, n=115$ clumps } \\
\hline All & $19.9 \pm 2.8$ & $2.4 \pm 3.3$ & $15.2 \pm 6.9$ & $26.2 \pm 33.7$ & $1.7 \pm 1.5$ & $1.8 \pm 0.2$ & $287.3 \pm 493.9$ & $16.8 \pm 7.9$ & $4.0 \pm 0.4$ & 94 & 115 \\
\hline PS-C & $19.9 \pm 2.1$ & $3.2 \pm 2.9$ & $21.2 \pm 7.0$ & $41.6 \pm 33.8$ & $1.5 \pm 1.1$ & $1.7 \pm 0.1$ & $409.7 \pm 376.8$ & $17.4 \pm 4.0$ & $4.2 \pm 0.3$ & 14 & 14 \\
\hline PS & $18.3 \pm 2.6$ & $4.7 \pm 6.2$ & $18.2 \pm 6.9$ & $48.8 \pm 66.5$ & $2.6 \pm 2.5$ & $1.8 \pm 0.2$ & $657.7 \pm 1005.4$ & $20.4 \pm 9.8$ & $4.1 \pm 0.3$ & 16 & 19 \\
\hline PRE & $20.3 \pm 2.9$ & $1.8 \pm 1.9$ & $13.1 \pm 5.8$ & $18.4 \pm 12.2$ & $1.8 \pm 1.4$ & $1.8 \pm 0.2$ & $180.6 \pm 194.7$ & $15.9 \pm 7.6$ & $4.0 \pm 0.4$ & 64 & 82 \\
\hline PS PS-C & $19.1 \pm 2.5$ & $4.1 \pm 5.1$ & $19.6 \pm 7.1$ & $45.7 \pm 55.2$ & $1.7 \pm 1.5$ & $1.8 \pm 0.1$ & $552.5 \pm 810.7$ & $19.1 \pm 8.0$ & $4.2 \pm 0.3$ & 30 & 33 \\
\hline
\end{tabular}

Notes. Mean temperature $T$, flux density $F_{v}$, column density $\left\langle N\left(\mathrm{H}_{2}\right)\right\rangle$, peak column density $N\left(\mathrm{H}_{2}\right)_{850}$, ratio of peak to mean $N\left(\mathrm{H}_{2}\right)$, mean beta, observed mass $M$, virial mass $M_{\text {vir }}$, and distance $d$ of unbound SL, PRE, and PS-C, and unconfirmed (PS) clumps. Only fields with distance estimates are considered. Average temperatures and column densities are further limited to fields with SPIRE data. The number of clumps of each type with distance estimates and Herschel SPIRE data are listed in Col. 11 and the total number of clumps with distance estimates in Col. 12. Estimates for all starless (SL plus PRE) and all protostellar (PS plus PS-C) clumps are also included.

G159.23-20.09, and G202.31+02.53. This sample contains 10 potentially protostellar clumps, of which one is confirmed. The other three clumps are unbound starless or prestellar. The line width was estimated from the spectra plotted in Appendix L. For the 13 clumps we calculate $\langle\Delta V\rangle=0.9 \pm 0.03 \mathrm{~m} \mathrm{~s}^{-1}$ and $\sigma_{\text {avg }}=$ $0.43 \pm 0.01 \mathrm{~km} \mathrm{~s}^{-1}$. As the line widths for the 13 clumps are similar enough, we use $\sigma_{\text {avg }}$ to estimate the critical virial mass for the rest of the clumps. This is very similar to the nonthermal velocity dispersion for dense clumps of $0.49 \mathrm{~km} \mathrm{~s}^{-1}$ derived by Juvela et al. (2018c). However, we note that this is a tentative classification because the local environment strongly affects the kinematics of a clump. For this reason, we also include values derived for all starless clumps in Table 5.

We classify starless clumps as prestellar if they are gravitationally bound, with $\alpha_{\text {vir }} \leq 1.0$. Through propagation of error we find that uncertainty in virial mass is approximately $100 \%$. Out of 234 starless clumps with distance estimates, we estimate 210 (90\%) to be virially bound. Extrapolated into the whole sample, virial analysis predicts $\sim 37$ unbound and $\sim 331$ prestellar clumps, although of course magnetic forces, for example, play a role in core evolution.

By requiring that YSOs have $160 \mu \mathrm{m}$ intensity significantly higher than $250 \mu \mathrm{m}$ intensity (Sect. 3.6.3), we find that 50 out of
$108(46 \%)$ YSOs that are located on Herschel PACS $160 \mu \mathrm{m}$ and SPIRE $250 \mu \mathrm{m}$ fields can be confirmed as YSOs. A further 106 $(51 \%)$ out of 214 total YSOs do not have Herschel $160 \mu \mathrm{m}$ and $250 \mu \mathrm{m}$ data. Only $242(46 \%)$ of all clumps have both SPIRE $250 \mu \mathrm{m}$ and PACS $160 \mu \mathrm{m}$ data, as well as mass estimates. Out of these, we find $28(12 \%)$ protostellar and 167 (69\%) starless clumps, out of which $145(60 \%)$ are classified prestellar. A further $47(19 \%)$ clumps are tentatively protostellar but without further confirmation from Herschel intensity ratio $\Delta C$.

Uncertainty in number of YSOs associated with a clump depends on a range of factors, including the completeness of the surveys used, the distance, the extinction, and the YSO class. We assume an uncertainty in the position across all catalogs of $6.3^{\prime \prime}$ (22\% uncertainty), a chance of misclassifying other sources as YSOs of $10 \%$, and of missing YSOs of $10 \%$, resulting in final uncertainty of $26 \%$ in number of YSOs.

\subsection{Clump properties}

We present the full catalog of derived clump properties in Appendix K. Mean temperature, flux density, opacity spectral index, mass, and mean and peak column density for all unbound starless (SL), prestellar (PRE), and protostellar (PS) clumps with 

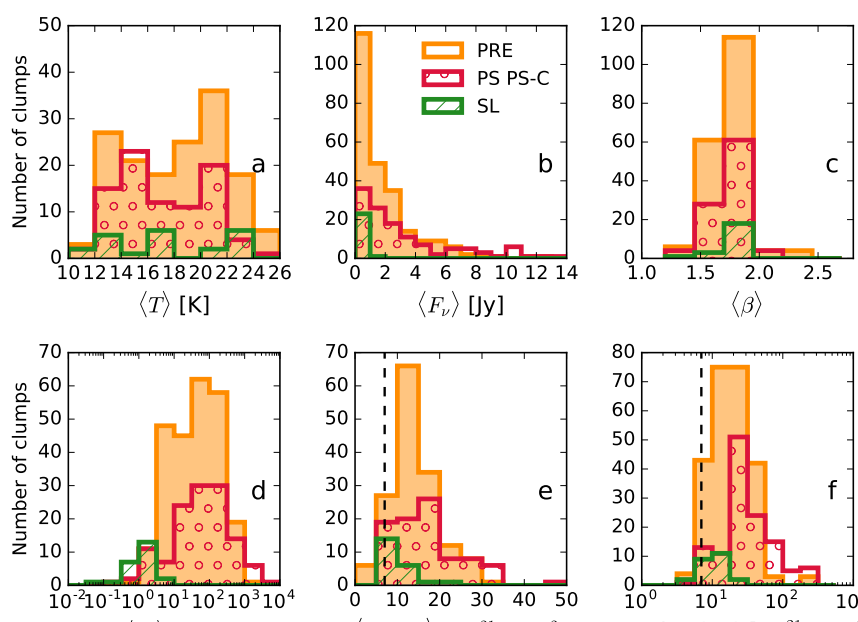

$\langle M\rangle\left[\mathrm{M}_{\odot}\right]$

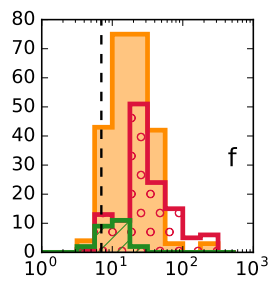

Peak $N\left(\mathrm{H}_{2}\right)\left[10^{21} \mathrm{~cm}^{-2}\right]$

Fig. 10. Histograms for properties of unbound starless (SL), prestellar (PRE), and all protostellar (PS PS-C) clumps. (a) Mean temperature. (b) Flux density. $(c)$ Mean clump opacity spectral index $(d)$ Mass, plotted on a logarithmic scale. (e) Mean clump column density. $(f)$ Peak clump column density. The dotted line in (e) and (f) correspond to $A_{v}=7$.

distance is shown in Table 5 and plotted in Fig. 10. The confirmed protostellar (PS-C) clumps are included under the PS bar in Fig. 10; but these are listed separately in Table 5, which also includes derived values for all starless (SL PRE) and all protostellar (PS PS-C) clumps.

We divided our clumps into four distance bins (bin I: $d<$ $0.5 \mathrm{kpc}$, bin II: $0.5 \leq d<1.5 \mathrm{kpc}$, bin III: $1.5 \leq d<3.0 \mathrm{kpc}$, and bin IV: $d \geq 3.0 \mathrm{kpc}$ ). We chose these distance bins because clump distances fall on four distinct peaks, shown in Fig. M.1. As a consequence of the large range of distances, we do not include analysis of regions with no distance estimates, which includes 193 clumps in 18 fields.

\subsubsection{Dust opacity spectral index in clumps}

Dust emission within clumps was examined based on the pixelby-pixel MBB fits of Herschel 250-500 $\mu \mathrm{m}$ data. The mean opacity spectral index over the clumps (Table 5, Col. 7) is lower by $\sim 0.2$ compared to the full fields. The opacity spectral index value does not significantly differ between the prestellar and starless clumps or between the distance bins (Fig. M.2). Overall, we find $\beta$ to be fairly consistent, with a standard deviation in all categories of $\leq 0.2$.

\subsubsection{Clump sizes}

Clump angular effective radii range from 1.7 to $49.4^{\prime \prime}$, with a mean of $(15.2 \pm 6.8)^{\prime \prime}$, and show no dependence on distance (Fig. M.3). Mean spatial effective radius is $(0.2 \pm 0.1) \mathrm{pc}$, with a range from $0.006-0.6 \mathrm{pc}$. As the uncertainties of linear size are dominated by distance, $30 \%$ uncertainties are adopted for the effective radius and the SCUBA-2 FWHM, 14.6", for angular radii. The uncertainty in linear size is discussed further in Sect. 5.4.2. The clump sizes are similar above the threshold of the SCUBA-2 beam size, most likely because of selection effects: large-scale structure is filtered out and smaller-scale structure is not resolved by SCUBA-2. The sizes of the unresolved small clumps are unreliable. There is, in general, no significant difference in the angular sizes of different clump categories.

The average area of a clump is approximately $0.25 \mathrm{arcmin}^{2}$. Clumps are generally round and have a mean aspect ratio of 0.75 . More massive clumps are also likely to be rounder, whereas smaller cores have a large variation in aspect ratio. This may be due to deconvolution of clumps close to beam size.

\subsubsection{Clump temperatures}

Based on 250-500 $\mu \mathrm{m}$ Herschel data, the average temperature over a clump ranges from 10 to $25 \mathrm{~K}$ and has a mean of $17.8 \pm 3.6 \mathrm{~K}$ over all clumps. The total error in Herschel intensities $I_{250}$ is estimated to be below $10 \%$, resulting in temperature errors of the order of $1 \mathrm{~K}$ as in Montillaud et al. (2015).

The temperature is strongly correlated with distance (Fig. M.4). In distance bins I and II, the mean temperature is under $16 \mathrm{~K}$, rising to almost $20 \mathrm{~K}$ in more distant bins. We do not see large differences in temperature between clump categories. The largest differences are found in bin II, in which there is a difference of $4.6 \mathrm{~K}$ between SL and PS-C clumps. In the largest distance bins, bins III and IV, PRE clumps have higher mean temperatures. PS clumps have generally higher temperature than PS-C clumps.

\subsubsection{Column density}

Based on $250-500 \mu \mathrm{m}$ data, mean column density over all the clumps is $(1.5 \pm 0.7) \times 10^{22} \mathrm{~cm}^{-2}$, corresponding to visual extinction $\left\langle A_{v}\right\rangle=15.0 \pm 7.0 \mathrm{mag}$, using the relation $N\left(\mathrm{H}_{2}\right) / A_{v}=$ $10^{21} \mathrm{~cm}^{-2} \mathrm{mag}^{-1}$ (Bohlin et al. 1978; Sadavoy et al. 2012). The uncertainty of the dust opacity $\kappa$ is at least $50 \%$, leading to a similar uncertainty in $N\left(\mathrm{H}_{2}\right)$. The highest column density is found in PS-C clumps in bin IV, whereas SL clumps are the most diffuse. The PRE clumps have lower column density than PS-C or PS clumps, also suggesting that these clumps include matter from the diffuse ISM. There is little change in column density as a function of distance (Fig. M.5).

For comparison, peak column densities were derived from the higher resolution SCUBA-2 surface brightness measurements. For clumps without $250-500 \mu \mathrm{m}$ temperature estimates (or with unrealistically low estimates below $10 \mathrm{~K}$ ), the mean clump temperature of $18 \mathrm{~K}$ was used. We plot SCUBA-2 peak column density against Herschel column density in Fig. 11a. Column density was estimated by calculating optical depth $\tau$ from Eq. (2) and column density using Eq. (3). We calculate volume density from Eq. (5). The average of clump peak column densities is $(3.0 \pm 0.2) \times 10^{22} \mathrm{~cm}^{-2}$, twice as high as that estimated from Herschel (Fig. 11a), with a maximum of $(3.1 \pm 1.6) \times 10^{23} \mathrm{~cm}^{-2}$. These values give a better picture of the densest regions of the clouds.

Highest peak column densities are found in protostellar clumps; PS-C clumps have the highest peak column density in bin II and PS clumps in other distance bins (Table 5). The SL clumps are the most diffuse, and PRE clumps have peak column densities over double that of SL clumps. There is a not much difference in mean peak column density over the distance bins. Average volume density calculated with peak column density $(\mathrm{Eq} .(5))$ is $(6.1 \pm 0.04) \times 10^{7} \mathrm{~cm}^{-3}$, and values range from $(1.1 \pm 0.7) \times 10^{4} \mathrm{~cm}^{-3}$ to $(1.3 \pm 0.8) \times 10^{9} \mathrm{~cm}^{-3}$ (Fig. $\left.11 \mathrm{~b}\right)$.

\subsubsection{Clump masses}

Clumps have a wide range of masses, from $0.04 M_{\odot}$ to $4259 M_{\odot}$ based on Herschel temperature and SCUBA-2 flux, with a mean mass of $\left(136_{-136}^{+316}\right) M_{\odot}$. Uncertainty in mass is dominated by 

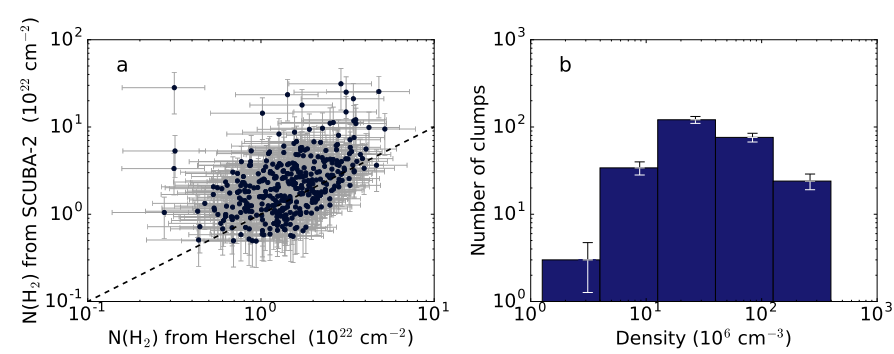

Fig. 11. (a) Column density from Herschel (x-axis) plotted against column density from SCUBA-2 peak values ( $y$-axis). The black dotted line shows where both are equal. The clumps for which temperature was assumed to be unrealistic, set to $18 \mathrm{~K}$, are denoted with red squares and the rest with dark blue circles. (b) Histogram of peak volume density of each clump with uncertainty.

distance, but is also affected by uncertainty in $\kappa, \beta$, and flux density. We assume a total uncertainty in mass of $80 \%$.

Mass increases significantly with distance (Fig. M.6), proving that the most distant clumps are really unresolved clouds, which likely contain many smaller clumps and cores. In all distance bins, SL clumps are the least massive by as much as a factor of 10 when compared to protostellar clumps in bin II. Protostellar clumps are the most massive across all bins; PS-C clumps are generally smaller than PS clumps. The masses of PRE clumps are of the same order as those of protostellar clumps.

Virial masses are generally smaller than observed masses, have a mean of $(9.8 \pm 7.4) M_{\odot}$, and a smaller range of $(0.3 \pm 0.5)-(38.5 \pm 53.9) M_{\odot}$. Few nearby cores are virially bound and no distant clouds are unbound. There is an increase of virial mass with distance by about one order of magnitude (Fig. M.7). As our ${ }^{13} \mathrm{CO}$ data comes from fields at distances of $\sim 1.7,0.8$, and $0.7 \mathrm{kpc}$, our virial masses are likely to be more precise in bin II, where our derived virial masses most closely match our observed masses. We derive a final uncertainty of $140 \%$ on virial mass.

\section{Discussion}

\subsection{Properties of the extended ISM}

Various relations between temperature and spectral index can be found in the literature, from the inner Galaxy (Paradis et al. 2010), the outer Galaxy (Désert et al. 2008), the solar neighborhood (Dupac et al. 2003), and the entire Galaxy (Planck Collaboration XI 2014). Even after accounting for error, anticorrelation has been found to exist at the scale of cold clouds and near the Galactic poles (e.g., Dupac et al. 2003; Désert et al. 2008; Paradis et al. 2010; Planck Collaboration XI 2014; Juvela et al. 2015); however, a positive correlation has been found in some Bok globules and the overall Galactic plane (Kelly et al. 2012; Planck Collaboration XI 2014), as well as in small, dense regions near strong point sources (Juvela et al. 2015). Our simulations strongly suggest that not all the anticorrelation is due to noise.

Our derived mean temperature of $18.8 \mathrm{~K}$ over the fields is slightly lower than all-sky models, and our mean column density approximately four times higher, as expected for star-forming regions, which are protected from the interstellar radiation field. In the literature, an average dust temperature of $19.7 \mathrm{~K}$ has been derived for the whole sky, which is dominated by diffuse regions (Planck Collaboration XI 2014). The mean temperatures of large-scale filaments in the Milky Way are approximately 19$21 \mathrm{~K}$, and have column densities of approximately $10^{21} \mathrm{~cm}^{-2}$ (Zucker et al. 2018). In the case of dense clumps, Juvela et al. (2015) find mean temperatures of $16.9 \mathrm{~K}$ for SPIRE-only data, and $18.4 \mathrm{~K}$ for fits including also SPIRE $160 \mu \mathrm{m}$. Juvela et al. (2015) also derive slightly lower temperatures from $250-500 \mu \mathrm{m}$ than from 160-500 $\mu \mathrm{m}$ Herschel data.

We derive a mean dust opacity spectral index of 1.9 over the fields using $250-500 \mu \mathrm{m}$ data and 1.7 by including also $160 \mu \mathrm{m}$ data. Juvela et al. (2015) derive slightly higher spectral index of 1.89 for 166 GCC fields, using Herschel and Planck data. The omission of shorter wavelengths increases the mean spectral index; in our sample there is a mean of $\Delta \beta \approx 0.06$. Juvela et al. (2015) find slightly larger difference; however, their sample includes a larger range of wavelengths.

Elia et al. (2017) find no relation between temperature and Galactocentric radius, but find a slight increase for median bolometric temperature. Juvela et al. (2015) also do not find significant latitude- or longitude-dependence on temperature, but find higher spectral index at higher latitudes. However, Marsh et al. (2017) find a decreasing radial profile of dust temperature using Herschel data, while Urquhart et al. (2018) find a mild decrease using SCUBA-2 observations. Many nearby spiral galaxies have mean cold dust temperatures of approximately $25 \mathrm{~K}$ near the center $(\ell \sim 0)$, which drops $\sim 10 \mathrm{~K}$ toward the edge of the galaxies (Tabatabaei et al. 2014; Meléndez et al. 2015; Riener et al. 2018). We also find lower temperatures away from the plane and center of the Galaxy, although not as pronounced; of course, the extragalactic surveys study the entire cold ISM and not the densest clumps. Eden et al. (2019) also find high column densities around $10^{22} \mathrm{~cm}^{-2}$ near the plane of the Galaxy for their observed SCOPE cores.

\subsection{YSO association}

There are several possibilities why over half of YSO candidates located within the SCUBA-2 fields are not associated with any clumps. First, they may be more evolved YSOs that have already left the densest cores; however, these would likely already be more evolved stars. Second, some cores may have lower flux and thus not have been found by the FellWalker algorithm. There are also clouds outside the clumps, although these typically have lower column densities and are generally not star-forming. In the case of large, smooth clumps, the filtering of large-scale structure may also prevent detection. Finally, some YSO candidates in the catalogs may have been incorrectly classified sources. In addition, as the YSO association is based on the match of projected positions on the sky, YSOs may not be located at the distance of the clumps. This is especially likely for fields near the plane of the Galaxy, which are likely to have several SF regions and IR sources along the line of sight.

\subsection{Clump classification}

We find mean virial mass of $9 M_{\odot}$, and mean $\alpha_{\text {vir }}$ of $\sim 0.5$, which is a lower virial mass and mean $\alpha_{\text {vir }}$ than found in Contreras et al. (2017) for clumps found within $10 \mathrm{kpc}$. Owing to increased difficulty of detecting fainter clumps, a larger percentage of distant starless clumps are classified as prestellar (Benedettini et al. 2018). According to the literature, mostly using Herschel observations, only $20 \%$ of starless cores were classified as prestellar in nearby clouds with distance under 200 pc (Marsh et al. 2016; Benedettini et al. 2018; Bresnahan et al. 2018; Tang et al. 2018). This percentage rises to nearly $60 \%$ at distances between 200 

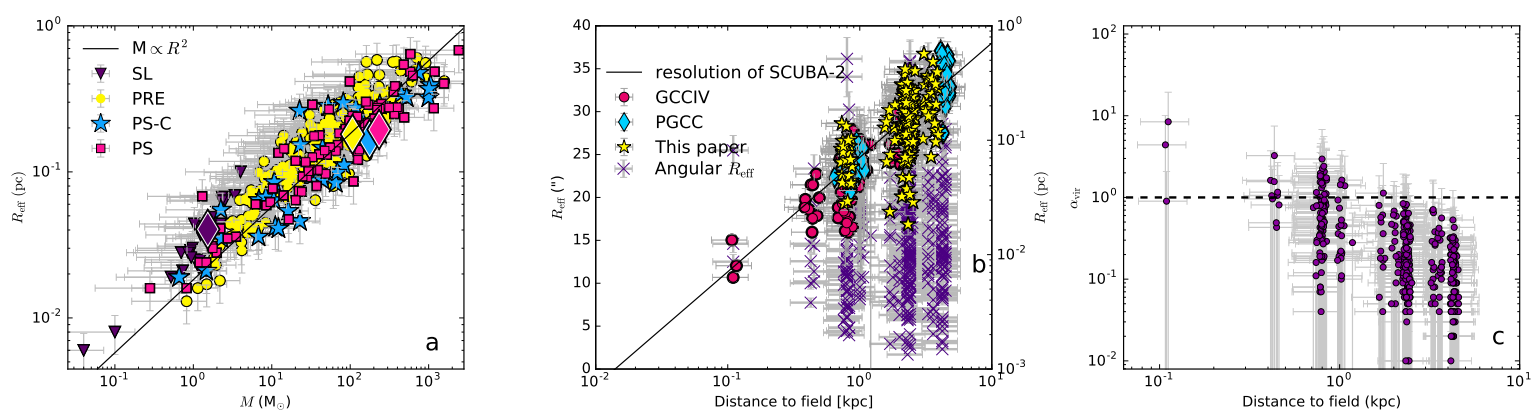

Fig. 12. (a) Effective clump radius as a function of clump mass. The symbols correspond to unconfirmed PS (pink squares), PS-C (blue stars), PRE (yellow circles), and unbound SL (purple triangles) clumps. Mean mass and radius are plotted with diamonds of the same color. The black line shows $M \propto R^{2}$. (b) Angular size (left axis, purple crosses) and spatial size (right axis) of the clumps as a function of distance on a logarithmic scale. The distances are from GCCIV (Montillaud et al. 2015) (pink circles), the PGCC catalog (Planck Collaboration XXVIII 2016) (blue diamonds), and this paper (yellow stars). Random jitter of $\leq 10 \%$ has been added to distances to improve readability. The black line corresponds to the resolution of the SCUBA-2 instrument. (c) Clump $\alpha_{\text {vir }}$ (blue circles) plotted as a function of distance. Random jitter of $\pm 3 \%$ of distance has been added for readability. The black dashed line is the limit of gravitational instability.

and 500 pc (Polychroni et al. 2013; Könyves et al. 2015) and is $80-100 \%$ at distances over $1 \mathrm{kpc}$ (Giannini et al. 2012; Elia et al. 2013; Merello et al. 2019). This trend is noted across a wide range of distances in Montillaud et al. (2015) and is also clearly visible in our data; Fig. 12c shows that $\alpha_{\text {vir }}$ is significantly lower, and thus clumps appear to be more gravitationally bound in distant fields. Furthermore, the smaller $\alpha_{\text {vir }}$ at larger distances may be caused by uncertainties in distance, as $\alpha_{\mathrm{vir}} \propto M_{\mathrm{obs}}^{-1} \propto d^{-1}$. Finally, resulting from our lack of dense gas tracers, we are unable to observe the kinematic environments of the clumps, and it is likely that we underestimated the turbulence within some regions.

Clumps classified as protostellar should be already undergoing SF. In addition to the possibility of misclassifying starless clumps as protostellar, quiescent cores classified as starless may have hidden SF, which is only visible through interferometric observations (Sanhueza et al. 2019; Li et al. 2019a). Many studies have used a detection in $70 \mu \mathrm{m}$ as a definitive sign of protostars (e.g., Giannini et al. 2012; Könyves et al. 2015; Merello et al. 2019) or evolved clumps (Giannetti et al. 2019). However, especially low-mass SF clumps have been found to be quiet in $70 \mu \mathrm{m}$ (Traficante et al. 2017), and some $70 \mu \mathrm{m}$-quiet clumps have been found to have associated water masers, a sign of SF activity (König et al. 2017).

In this study, we looked for additional evidence for protostellar clumps by looking at the increase in the $160-250 \mu \mathrm{m}$ ratio. With this clarification, we find that just under one half of the YSOs can be confirmed. However, Montillaud et al. (2019) find that dust temperature often also drops toward the center of protostellar clumps. Furthermore, they find several "warm starless cores", finding that cores without SF can also show significant increase in temperature toward the center. Their sample was at a distance of $760 \mathrm{pc}$ and the $160-250 \mu \mathrm{m}$ ratio might be a more sensitive test of the protostellar status in more nearby fields. We perform this test only on those clumps with a candidate YSO from one or more catalogs, and thus are less likely to misclassify warm starless cores as protostellar. Warm starless cores are also often more diffuse, and given the requirement of a SCUBA2 detection and thus high column density of all our sources, it is unlikely that our sample includes warm starless cores misclassified as protostellar clumps.

\subsection{Clump properties}

Figure M.1 shows histograms and the correlations of clump distance, aspect ratio, mass, temperature, column density, and volume density (Fig. M.1). A strong correlation $(|r| \geq 0.4)$ is found between mass and distance, temperature and distance, volume density and distance, and temperature and volume density. A weak correlation $(0.2 \leq|r| \leq 0.4)$ is found between mass and the following quantities: temperature, column density, volume density, and aspect ratio.

We plot mass against $R_{\text {eff }}$, physical and angular size against distance, and $\alpha_{\text {vir }}$ against distance in Figs. 12a-c, respectively. The strong relation between distance and other parameters is apparent in the images.

\subsubsection{Clump spectral index}

The derived opacity spectral index of the clumps in our samples are in the range 1.3-2.4, with a mean around 1.7 using 250-500 $\mu \mathrm{m}$ data. Our sample agrees with current understanding of dense SF regions, which generally are understood to have $\beta \approx 1.8$ (Planck Collaboration XXII 2011; Planck Collaboration XXIII 2011; Planck Collaboration XXV 2011; Juvela et al. 2015). Our values are also consistent with those found in the $\lambda$ Orionis cloud of $\beta \simeq 1.65$, and slightly lower than those found in Orion A and Orion B (Yi et al. 2018). In the literature, the highest values of opacity spectral index have been found in SL clumps (Juvela et al. 2015). Although all averages of spectral index in our sample are similar within the uncertainties, our SL clumps also show slightly higher mean values. Juvela et al. (2015) find values up to $\beta=2.2$ using the full sample of GCC clumps.

We extended the SED analysis of our clumps to $850 \mu \mathrm{m}$, which showed a slight increase in opacity spectral index as a function of wavelength. This is in contrast to previous studies, in which opacity spectral index generally decreases beyond $500 \mu \mathrm{m}$ (Reach et al. 1995; Paradis et al. 2012; Juvela et al. 2015). We also do not find any $850 \mu \mathrm{m}$ excess, but rather a slight loss around $5 \%$.

\subsubsection{Clump sizes}

There is a correlation between distance and derived radius, both in the literature and in our sample, with a derived relation of $R_{\text {eff }}[\mathrm{pc}]=7.1 \times d[\mathrm{kpc}]+1.0$. This relation is set primarily by the resolution of the SCUBA-2 instrument on the lower end, and spatial filtering on the upper end. The clump angular effective radius is similar for all clumps regardless of distance (Fig. 12b). 

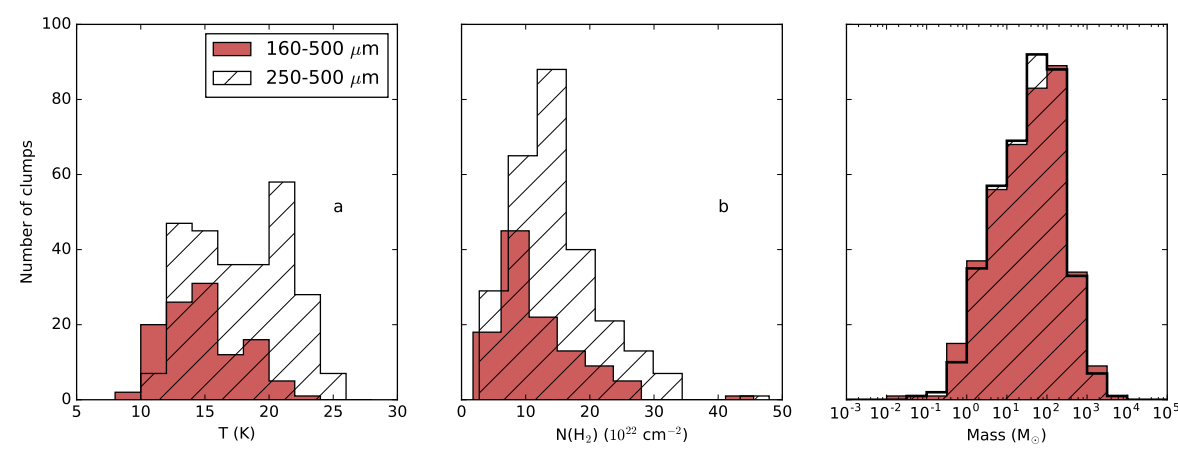

Fig. 13. Histograms of $(a)$ mean clump temperature, $(b)$ mean column density, and $(c)$ mass calculated using 250-500 $\mu \mathrm{m}$ data (black dashes) and $160-500 \mu \mathrm{m}$ data (pink) using a constant $\beta=1.8$. Clumps are not separated by type or distance for this analysis. Mass estimation assumes $d=1 \mathrm{kpc}$ and $F_{850}=2 \mathrm{Jy}$ for clumps without these values.
The black line represents the resolution of the SCUBA-2 instrument; the deconvolved sizes of some clumps are below the beam size. Typical core sizes in the literature are $\sim 0.01 \mathrm{pc}$ at a distance of $200 \mathrm{pc}$ (Sadavoy et al. 2012), but rise to $\sim 0.1 \mathrm{pc}$ at distances over $5 \mathrm{kpc}$ (Contreras et al. 2017; Liu et al. 2018a; Merello et al. 2019). Our derived radii and distances match the literature. In addition, more massive clumps are also likely to be rounder, whereas smaller cores have a large variation in aspect ratio. As in our sample, the peak aspect ratio of all SCOPE sources is also close to 1 compared to all PGCC sources, which are more extended or filamentary (Eden et al. 2019). However, many of our clumps fall on filamentary structures, as can be seen from the SCUBA-2 surface brightness maps in Appendix J.

\subsubsection{Clump temperatures}

The mean clump temperature in our study of $17.8 \pm 3.6 \mathrm{~K}$ is similar to the mean temperatures of 3000 MALT90 highmass clumps at distances from 3-10 kpc (Guzmán et al. 2015; Contreras et al. 2017). The temperatures of $15.5 \pm 3.5 \mathrm{~K}$ in clumps with $d<1.5 \mathrm{kpc}$ (distance bins I and II) are consistent with those found within nearby regions (e.g., Corona Australis and Cepheus; Bresnahan et al. 2018; Pattle et al. 2017b) and all PGCCs and other GCC studies (Juvela et al. 2018b; Eden et al. 2019), but a few degrees higher than those found in the Lupus cluster and some infrared dark clouds (Sanhueza et al. 2013, 2017; Traficante et al. 2015; Benedettini et al. 2018). In the literature prestellar clumps have been found to be colder than protostellar clumps (Guzmán et al. 2015; Montillaud et al. 2015; Contreras et al. 2017; Elia et al. 2017; Merello et al. 2019). In our sample, the mean clump temperatures are generally equal within their uncertainties. The peak temperatures of a few clumps are over $20 \mathrm{~K}$, suggesting presence of YSO formation. As our sample derives from the Planck cold clumps sample, we do not see temperatures as high as those found in, for example, the MALT90 sample (Guzmán et al. 2015).

A strong increase in temperature as a function of distance is visible in our sample. The mean dust temperature increases with distance as a consequence of the difficulty in observing distant colder sources, as simulated by Montillaud et al. (2015). Furthermore, nearby fields are likely to be dominated by a single dense core, whereas the clumps detected in distant fields are clouds that include also more diffuse, warmer material (Juvela et al. 2015). Temperature within ATLASGAL clumps has been found to increase with increasing helio- and galactocentric distance (Elia et al. 2017; Urquhart et al. 2018), similar to our sample. As lineof-sight temperature variations tend to increase the measured color temperatures (Montillaud et al. 2015), it is likely that this analysis overestimates the physical mass-averaged dust temperature. This effect gets worse with the inclusion of shorter wave- lengths such as PACS $160 \mu \mathrm{m}$ estimates (Shetty et al. 2009a,b; Juvela et al. 2018b), thus using only SPIRE wavelengths may give a less biased estimate. We calculated temperatures, column densities, and masses for clumps using 160-500 $\mu \mathrm{m}$ data (Fig. 13). The mean temperatures are $\sim 3 \mathrm{~K}$ lower and the mean column densities $\sim 3 \times 10^{21} \mathrm{~cm}^{-2}$ lower than those derived using 250-500 $\mu \mathrm{m}$ data. Assuming a distance of $1 \mathrm{kpc}$ and a flux of $2 \mathrm{Jy}$, clump masses derived using $160-500 \mu \mathrm{m}$ data are $\sim 4 M_{\odot}$ higher.

\subsubsection{Column density}

The mean column density over the extracted clumps, calculated from Herschel data is approximately $(1.5 \pm 0.7) \times 10^{22} \mathrm{~cm}^{-2}$, and is highest for protostellar clumps. The peak column densities calculated from SCUBA-2 $850 \mu \mathrm{m}$ intensities are higher by about $10 \%$ for SL clumps and almost three times for PS-C clumps (Sect. 4.8.4). The column densities derived for our sample are higher than those found by other studies using only Herschel data (e.g., di Francesco et al. 2010; Könyves et al. 2015; Guzmán et al. 2015; Benedettini et al. 2018) because SCUBA-2 targets are selected for high column density. We also find significantly higher volume density than in Contreras et al. (2017), where our derived median volume density, derived from peak column density, is larger than their maximum $n\left(\mathrm{H}_{2}\right)$. Contreras et al. (2017) also find volume density decreasing with increased dust temperature; while this trend is also visible in our results it is not significant. As these sources may be more extended along the line of sight than the distance corresponding to a single SCUBA-2 beam, our method likely overestimates the mean volume density. Especially in highly crowded regions near the plane of the Galaxy, it is possible for irregularly-shaped diffuse clouds to overlap along the line of sight, creating an illusion of a single dense clump. Even isolated clumps may face this issue because the random orientation of irregular 3D structures may affect derived clump properties. These cloud structures require observation of $3 \mathrm{D}$ chemistry, kinematics, and polarization to resolve (Ge et al. 2020). However, while high-density prestellar cores exist (e.g., L1544; Ohashi et al. 1999), these high values suggest the sources have the potential to be directly involved in SF.

A density threshold for SF was first proposed by Schmidt (1959) and has been observed (Evans et al. 2009; Heiderman et al. 2010; Lada et al. 2010, 2012). Using $A_{v, \mathrm{BG}}=7 \mathrm{mag}$ as the threshold for SF (Clark \& Glover 2014; Könyves et al. 2015), approximately $88 \%$ of PS-C, $85 \%$ of PS, $100 \%$ of PRE, and $87 \%$ of SL clumps with estimates for column density are found above this threshold; however, these percentages apply to the mean clump column densities. In our sample, we thus do not detect significant differences between clump categories, similar to Merello et al. (2019). Commonly, in the literature prestellar cores are associated with high background column density. About $90 \%$ 
of prestellar cores observed with Herschel in the Aquila complex are found in regions with $A_{v} \geq 7$, and all prestellar cores in Taurus on filaments with $A_{v} \geq 5$ (Könyves et al. 2015; Marsh et al. 2016). A similar high fraction of prestellar cores with high column density has been found in Corona Australis (Bresnahan et al. 2018).

In our sample, the ratio between the peak and mean column density ranges from $0.4-69$, where $90 \%$ of ratios are between $0.5-4.8$; this is a wider range than that found for the 3000 MALT90 clumps of Guzmán et al. (2015). We also find that prestellar clumps have the steepest column density profile, unlike, for example, Guzmán et al. (2015), who find the steepest profiles in protostellar clumps.

\subsubsection{Clump masses}

In our sample, we derive masses between 0.04-4259 $M_{\odot}$. Within all but bin II, prestellar clumps have larger masses; however, the overall distribution is dominated by the distance dependence. The mass of clumps compared to their radius is plotted for unbound starless, prestellar, and protostellar clumps in Fig. 12a, and this shows a strong linear correlation caused by the finite angular resolution of the SCUBA-2 instrument. The clump masses derived by also including $850 \mu \mathrm{m}$ data (Table B.1) are $\sim 15 \%$ higher, but show similar patterns to masses derived using only SPIRE data.

In previous Herschel studies, typical core masses of under $0.2 M_{\odot}$ have been found in the Lupus and Perseus MCs at a distance of $200 \mathrm{pc}$ (Benedettini et al. 2018; Sadavoy et al. 2012), and under $10 M_{\odot}$ in the Aquila complex at $300 \mathrm{pc}$ (Könyves et al. 2015). At up to $4 \mathrm{kpc}$ distances, significantly larger masses between 27 and $2000 M_{\odot}$ were found (Liu et al. 2018a); in a sample of Hi-GAL clumps at distances under $18 \mathrm{kpc}$, the mean mass was $850 M_{\odot}$ (Merello et al. 2019), and in a sample of MALT90 clumps at distances under $10 \mathrm{kpc}$, the mean mass was on the order of $10^{3} M_{\odot}($ Contreras et al. 2017). Higher masses are found toward the center of the Galaxy, as in a sample of 48 Planck clumps, no sources with $M>10^{4} M_{\odot}$ were found at Galactocentric distances over $6 \mathrm{kpc}$ (Zahorecz et al. 2016); sources closer to the Galactic center have larger heliocentric distances. As in our study, Hi-GAL protostellar clumps were found to have higher mass and density than prestellar clumps (Merello et al. 2019).

Distant starless clumps are more likely to be classified as prestellar than unbound because unbound clumps are more difficult to detect owing to lower flux (Benedettini et al. 2018). Within 500 pc of the Sun, 20-60\% of starless sources have been found to be prestellar (Polychroni et al. 2013; Könyves et al. 2015; Marsh et al. 2016; Benedettini et al. 2018; Bresnahan et al. 2018; Tang et al. 2018), although mostly based on Herschel studies. At distances of $\sim 1 \mathrm{kpc}, 80-100 \%$ of starless sources are prestellar (Giannini et al. 2012; Elia et al. 2013). This of course depends strongly on clump detection methods and on the sensitivity of the observations.

The derived characteristics of our sources match these previous studies very well. In general, clumps in nearby fields under $0.5 \mathrm{kpc}$ have mass under $5 M_{\odot}$ and radius under $0.1 \mathrm{pc}$, fitting the definition of cores. The most distant clumps are likely entire bound clouds and have radii nearer to $1 \mathrm{pc}$ and mass up to $4 \times 10^{3} M_{\odot}$, which themselves contain unresolved clumps and cores. We also note this strong correlation between virial $\alpha$ and distance, seen in Fig. 12, where all clumps with $d \gtrsim 2 \mathrm{kpc}$ are classified as gravitationally bound.

\section{Summary and conclusions}

We have characterized 529 dense clumps selected from the JCMT SCOPE survey. The clumps were selected from 53 fields mapped by the Herschel satellite and cover a range of distances from 0.1 to $4.5 \mathrm{kpc}$. We classify the clumps as protostellar, prestellar, or unbound starless based on their estimated virial masses, the presence of YSOs, and dust temperature (Sect. 4.7). We find the following properties for these dense clumps.

1. The gravitational stability was estimated for 336 clumps with distance estimates. A total of 242 (46\%) clumps have both mass estimates and measurements of the Herschel $160 \mu \mathrm{m} / 250 \mu \mathrm{m}$ intensity ratio.

(a) Using those 242 clumps, we find 167 (69\%) starless clumps, out of which 145 are classified as prestellar, and $75(31 \%)$ protostellar clumps (PS), 28 of which we are able to further confirm by an increase in the Herschel $160 \mu \mathrm{m}$ versus $250 \mu \mathrm{m}$ intensity ratio.

(b) The reliability of the classification is affected by the lack of measurements of molecular line data (for estimates of turbulent support and external pressure) and magnetic fields.

(c) We find that many prestellar clumps are likely to be entire bound structures, which themselves contain smaller clumps and cores. This is because of their significantly higher mean distance compared to the other categories.

2. Gravitational stability is also dependent on distance; all clumps beyond $2 \mathrm{kpc}$ are classified as gravitationally bound. These fields have high column density and low temperature, and there is little effect of internal heating. The characteristics of these sources show strong dependence on distance. They span a wide range of objects, from individual nearby high-latitude cores to distant star-forming clouds near the Galactic center. Calculated distances to sources can be improved in the future by utilizing new observations, such as Gaia data. We do not find it possible to resolve significant differences between the various categories of clumps with our data. Chemical evolution surveys are necessary to confirm the evolutionary status of these sources.

The mean temperature of dense clumps is $17.8 \mathrm{~K}$, where clumps with distance $d<1.5 \mathrm{kpc}$ have $\langle T\rangle \simeq 15.5 \mathrm{~K}$ and more distant clumps have $\langle T\rangle \simeq 19.4 \mathrm{~K}$. All starless (SL PRE) clumps have temperatures higher by $1 \mathrm{~K}$ than all protostellar (PS PS-C) clumps, but also have higher mean distances by $\sim 0.4 \mathrm{kpc}$. We attribute the strong temperature dependence on distance to three factors: the increasing difficulty of detecting distant cold sources, the fact that these distant fields are closer to the Galactic center, and that distant sources cover a larger spatial area and thus include more diffuse matter.

The clump masses range from 0.04 to $4259 M_{\odot}$. However, the mean virial mass only increases by one order of magnitude, leading to a higher percentage of bound sources in distant fields. The mean column density over the extracted clumps, calculated from Herschel data, is $(1.5 \pm 0.7) \times 10^{22} \mathrm{~cm}^{-2}$. The peak column densities calculated from SCUBA-2 $850 \mu \mathrm{m}$ intensities are approximately two times higher (Sect. 4.8.4), and the densest clumps have a peak column density of $\sim 10^{23} \mathrm{~cm}^{-2}$. Prestellar clumps tend to have the steepest column density profiles and unbound starless clumps have the flattest. Clump opacity spectral index shows no dependence on distance or the SF state of the clump, with the mean $\beta=1.7 \pm 0.1$. We find that among dense clumps the assumption of a constant opacity spectral index can be accurate.

We have characterized the dust properties of the ISM in the fields containing the SCUBA-2 clumps. Both the temperature 
and column density are higher in the plane of the Galaxy and drop toward $|b|=10^{\circ}$. We also find higher $T$ and $N\left(\mathrm{H}_{2}\right)$ near the Galactic center. Monte Carlo simulations suggest that only part of the detected $T-\beta$ anticorrelation is caused by observational noise. We derive a temperature $\beta$ relation of

$\beta=7.01 \times T^{-0.47}$,

for the temperature range $10-30 \mathrm{~K}$.

Acknowledgements. We thank the anonymous referee for their helpful comments. E. M. and M. J. acknowledge the support of the Academy of Finland Grant No. 285769. E. M. is funded by the University of Helsinki doctoral school in particle physics and universe sciences (PAPU). L. B. acknowledges support from CONICYT project Basal AFB-170002. J. H. thanks the National Natural Science Foundation of China under grant Nos. 11873086 and U1631237 and support by the Yunnan Province of China (No.2017HC018). This work is sponsored in part by the Chinese Academy of Sciences (CAS) through a grant to the CAS South America Center for Astronomy (CASSACA) in Santiago, Chile. P. S. was financially supported by a Grant-in-Aid for Scientific Research (KAKENHI Number 18H01259) of Japan Society for the Promotion of Science (JSPS). A. S. acknowledges financial support from the National Science Foundation grant AST-1715876. CWL is supported by Basic Science Research Program through the National Research Foundation of Korea (NRF) funded by the Ministry of Education, Science and Technology (NRF-2019R1A2C1010851). Tie Liu acknowledges the support from the international partnership program of Chinese academy of sciences through grant No. 114231KYSB20200009 and the support from National Natural Science Foundation of China (NSFC) through grant NSFC No.12073061. The James Clerk Maxwell Telescope is operated by the East Asian Observatory on behalf of The National Astronomical Observatory of Japan; Academia Sinica Institute of Astronomy and Astrophysics the Korea Astronomy and Space Science Institute; Center for Astronomical Mega-Science (as well as the National Key R\&D Program of China with No. 2017YFA0402700). Additional funding support is provided by the Science and Technology Facilities Council of the United Kingdom and participating universities in the United Kingdom and Canada. Additional funds for the construction of SCUBA-2 were provided by the Canada Foundation for Innovation. SCUBA-2 data were collected under program IDs M16AL003 and M15AI05. M16AL003 corresponds to the SCOPE survey, M15AI05 to data collected during the pilot program. This research used the facilities of the Canadian Astronomy Data Centre operated by the National Research Council of Canada with the support of the Canadian Space Agency. The James Clerk Maxwell Telescope is operated by the Joint Astronomy Centre on behalf of the Science and Technology Facilities Council of the United Kingdom, the Netherlands Organisation for Scientific Research, and the National Research Council of Canada.

\section{References}

Andre, P., Ward-Thompson, D., \& Barsony, M. 2000, Protostars and Planets IV, 59

André, P., Men'shchikov, A., Bontemps, S., et al. 2010, A\&A, 518, L102

André, P., Di Francesco, J., Ward-Thompson, D., et al. 2014, Protostars and Planets VI, 27

Arzoumanian, D., André, P., Didelon, P., et al. 2011, A\&A, 529, L6

Basu, A., \& Roy, S. 2013, MNRAS, 433, 1675

Beckwith, S. V. W., Sargent, A. I., Chini, R. S., \& Guesten, R. 1990, AJ, 99, 924

Benedettini, M., Pezzuto, S., Schisano, E., et al. 2018, A\&A, 619, A52

Berry, D. S. 2015, Astron. Comput., 10, 22

Beuther, H., Tackenberg, J., Linz, H., et al. 2012, ApJ, 747, 43

Bintley, D., Holland, W. S., MacIntosh, M. J., et al. 2014, Proc. SPIE, 9153, 915303

Bohlin, R. C., Savage, B. D., \& Drake, J. F. 1978, ApJ, 224, 132

Bohren, C. F., \& Huffman, D. R. 1998, Absorption and Scattering of Light by Small Particles (Wiley)

Boudet, N., Mutschke, H., Nayral, C., et al. 2005, ApJ, 633, 272

Brand, J., \& Blitz, L. 1993, A\&A, 275, 67

Bresnahan, D., Ward-Thompson, D., Kirk, J. M., et al. 2018, A\&A, 615, A125

Carey, S. J., Noriega-Crespo, A., Mizuno, D. R., et al. 2009, PASP, 121, 76

Chapin, E. L., Berry, D. S., Gibb, A. G., et al. 2013, MNRAS, 430, 2545

Cheng, Y., Tan, J. C., Liu, M., et al. 2018, ApJ, 853, 160

Chung, E. J., Lee, C. W., Kim, S., et al. 2019, ApJ, 877, 114

Clark, P. C., \& Glover, S. C. O. 2014, MNRAS, 444, 2396

Clemens, D. P. 1985, ApJ, 295, 422

Compiègne, M., Verstraete, L., Jones, A., et al. 2011, A\&A, 525, A103

Contreras, Y., Rathborne, J. M., Guzman, A., et al. 2017, MNRAS, 466, 340
Crutcher, R. M. 2012, ARA\&A, 50, 29

Cutri, R. M., Wright, E. L., Conrow, T., et al. 2012, Explanatory Supplement to the WISE All-Sky Data Release Products, Tech. rep.

Dempsey, J. T., Friberg, P., Jenness, T., et al. 2013, MNRAS, 430, 2534

Désert, F. X., Macías-Pérez, J. F., Mayet, F., et al. 2008, A\&A, 481, 411 di Francesco, J., Sadavoy, S., Motte, F., et al. 2010, A\&A, 518, L91 Dupac, X., Bernard, J. P., Boudet, N., et al. 2003, A\&A, 404, L11

Eden, D. J., Moore, T. J. T., Plume, R., et al. 2017, MNRAS, 469, 2163

Eden, D. J., Liu, T., Kim, K.-T., et al. 2019, MNRAS, 485, 2895

Elia, D., Molinari, S., Fukui, Y., et al. 2013, ApJ, 772, 45

Elia, D., Molinari, S., Schisano, E., et al. 2017, MNRAS, 471, 100 Enoch, M. L., Glenn, J., Evans, N. J., II, et al. 2007, ApJ, 666, 982 Evans, N. J. I., Dunham, M. M., Jørgensen, J. K., et al. 2009, ApJS, 181, 321 Exter, K. 2017, Quick-start guide to Herschel-PACS: the photometer, 1st edn. (Herschel Space Observatory)

Figueira, M., López-Calderón, C., Bronfman, L., et al. 2019, A\&A, 623, A141 Fontani, F., Commerçon, B., Giannetti, A., et al. 2018, A\&A, 615, A94 Gaczkowski, B., Preibisch, T., Ratzka, T., et al. 2013, A\&A, 549, A67

Ge, J. X., Mardones, D., He, J. H., et al. 2020, ApJ, 891, 36

Gholipour, M. 2019, MNRAS, 487, 3631

Giannetti, A., Bovino, S., Caselli, P., et al. 2019, A\&A, 621, L7

Giannini, T., Elia, D., Lorenzetti, D., et al. 2012, A\&A, 539, A156

Goldsmith, P. F. 2001, ApJ, 557, 736

Griffin, M. J., Abergel, A., Abreu, A., et al. 2010, A\&A, 518, L3

Gutermuth, R. A., \& Heyer, M. 2015, AJ, 149, 64

Guzmán, A. E., Sanhueza, P., Contreras, Y., et al. 2015, ApJ, 815, 130

Hacar, A., Tafalla, M., Kauffmann, J., \& Kovács, A. 2013, A\&A, 554, A55

Hall, H., \& Battersby, C. 2019, Am. Astron. Soc. Meeting Abstr., 233, 253.08

Heiderman, A., Evans, N. J. I., Allen, L. E., Huard, T., \& Heyer, M. 2010, ApJ, 723, 1019

Heiles, C., \& Troland, T. H. 2005, ApJ, 624, 773

Hennemann, M., Motte, F., Schneider, N., et al. 2012, A\&A, 543, L3

Holland, W. S., Bintley, D., Chapin, E. L., et al. 2013, MNRAS, 430, 2513

Johnstone, D., Ciccone, S., Kirk, H., et al. 2017, ApJ, 836, 132

Juvela, M., \& Ysard, N. 2012, A\&A, 541, A33

Juvela, M., Ristorcelli, I., Pelkonen, V. M., et al. 2011, A\&A, 527, A111

Juvela, M., Malinen, J., \& Lunttila, T. 2012, A\&A, 544, A141

Juvela, M., Montillaud, J., Ysard, N., \& Lunttila, T. 2013, A\&A, 556, A63

Juvela, M., Demyk, K., Doi, Y., et al. 2015, A\&A, 584, A94

Juvela, M., Guillet, V., Liu, T., et al. 2018a, A\&A, 620, A26

Juvela, M., He, J., Pattle, K., et al. 2018b, A\&A, 612, A71

Juvela, M., Malinen, J., Montillaud, J., et al. 2018c, A\&A, 614, A83

Kainulainen, J., Ragan, S. E., Henning, T., \& Stutz, A. 2013, A\&A, 557, A120

Kataza, H., Alfageme, C., Cassatella, A., et al. 2010, AKARI/IRC All-Sky

Survey Point Source Catalogue Version 1.0, http://www.ir.isas. jaxa.

jp/AKARI/Archive/Catalogues/PSC/RN/AKARI-IRC_PSC_V1_RN.pdf

Kelly, B. C., Shetty, R., Stutz, A. M., et al. 2012, ApJ, 752, 55

Kendrew, S., Simpson, R., Bressert, E., et al. 2012, ApJ, 755, 71

Kim, G., Tatematsu, K., Liu, T., et al. 2020, ApJS, 249, 33

Kong, S. 2019, ApJ, 873, 31

König, C., Urquhart, J. S., Csengeri, T., et al. 2017, A\&A, 599, A139

Könyves, V., André, P., Men'shchikov, A., et al. 2015, A\&A, 584, A91

Kruegel, E., \& Siebenmorgen, R. 1994, A\&A, 288, 929

Lada, C. J., Lombardi, M., \& Alves, J. F. 2010, ApJ, 724, 687

Lada, C. J., Forbrich, J., Lombardi, M., \& Alves, J. F. 2012, ApJ, 745, 190

Larson, R. B. 2003, Rep. Prog. Phys., 66, 1651

Li, H.-B., Yuen, K. H., Otto, F., et al. 2015, Nature, 520, 518

Li, X., Esimbek, J., Zhou, J., et al. 2019a, MNRAS, 487, 1517

Li, S., Zhang, Q., Pillai, T., et al. 2019b, ApJ, 886, 130

Li, C., Wang, H.-C., Wu, Y.-W., Ma, Y.-H., \& Lin, L.-H. 2020, Res. Astron. Astrophys., 20, 031

Liu, T., Wu, Y., Zhang, H., \& Qin, S.-L. 2012, ApJ, 751, 68

Liu, H.-L., Li, J.-Z., Wu, Y., et al. 2016, ApJ, 818, 95

Liu, T., Li, P. S., Juvela, M., et al. 2018a, ApJ, 859, 151

Liu, T., Kim, K.-T., Juvela, M., et al. 2018b, ApJS, 234, 28

MacLaren, I., Richardson, K. M., \& Wolfendale, A. W. 1988, ApJ, 333, 821

Malinen, J., Juvela, M., Rawlings, M. G., et al. 2012, A\&A, 544, A50

Marsh, K. A., Kirk, J. M., André, P., et al. 2016, MNRAS, 459, 342

Marsh, K. A., Whitworth, A. P., Lomax, O., et al. 2017, MNRAS, 471, 2730

Marton, G., Tóth, L. V., Paladini, R., et al. 2016, MNRAS, 458, 3479

Marton, G., Calzoletti, L., Perez Garcia, A. M., et al. 2017, ArXiv e-prints [arXiv:1705.05693]

Maruta, H., Nakamura, F., Nishi, R., Ikeda, N., \& Kitamura, Y. 2010, ApJ, 714, 680

Mason, B., Dicker, S., Sadavoy, S., et al. 2020, ApJ, 893, 13

Mattern, M., Kainulainen, J., Zhang, M., \& Beuther, H. 2018, A\&A, 616, A78

McKee, C. F., \& Ostriker, E. C. 2007, ARA\&A, 45, 565

Meléndez, M., Veilleux, S., Martin, C., et al. 2015, ApJ, 804, 46 
Mennella, V., Brucato, J. R., Colangeli, L., et al. 1998, ApJ, 496, 1058 Men'shchikov, A., André, P., Didelon, P., et al. 2010, A\&A, 518, L103 Meny, C., Gromov, V., Boudet, N., et al. 2007, A\&A, 468, 171

Merello, M., Molinari, S., Rygl, K. L. J., et al. 2019, MNRAS, 483, 5355

Molinari, S., Swinyard, B., Bally, J., et al. 2010, PASP, 122, 314

Montillaud, J., Juvela, M., Rivera-Ingraham, A., et al. 2015, A\&A, 584, A92

Montillaud, J., Juvela, M., Vastel, C., et al. 2019, A\&A, 631, L1

Motte, F., Andre, P., \& Neri, R. 1998, A\&A, 336, 150

Ngoumou, J., Hubber, D., Dale, J. E., \& Burkert, A. 2015, ApJ, 798, 32

Offner, S. S. R., Clark, P. C., Hennebelle, P., et al. 2014, in Protostars and Planets VI, eds. H. Beuther, R. S. Klessen, C. P. Dullemond, T. Henning, et al. (Tucson, AZ, USA: University of Arizona Press)

Ohashi, N., Lee, S. W., Wilner, D. J., \& Hayashi, M. 1999, ApJ, 518, L41

Ossenkopf, V., \& Henning, T. 1994, A\&A, 291, 943

Palmeirim, P., André, P., Kirk, J., et al. 2013, A\&A, 550, A38

Palmeirim, P., Zavagno, A., Elia, D., et al. 2017, A\&A, 605, A35

Paradis, D., Veneziani, M., Noriega-Crespo, A., et al. 2010, A\&A, 520, L8

Paradis, D., Paladini, R., Noriega-Crespo, A., et al. 2012, A\&A, 537, A113

Parikka, A., Juvela, M., Pelkonen, V.-M., Malinen, J., \& Harju, J. 2015, A\&A, 577, A69

Pattle, K., Ward-Thompson, D., Kirk, J. M., et al. 2015, MNRAS, 450, 1094

Pattle, K., Ward-Thompson, D., Berry, D., et al. 2017a, ApJ, 846, 122

Pattle, K., Ward-Thompson, D., Kirk, J. M., et al. 2017b, MNRAS, 464, 4255

Pilbratt, G. L., Riedinger, J. R., Passvogel, T., et al. 2010, A\&A, 518, L1

Planck Collaboration XXII. 2011, A\&A, 536, A22

Planck Collaboration XXIII. 2011, A\&A, 536, A23

Planck Collaboration XXV. 2011, A\&A, 536, A25

Planck Collaboration XI. 2014, A\&A, 571, A11

Planck Collaboration XXVIII. 2016, A\&A, 594, A28

Poglitsch, A., Waelkens, C., Geis, N., et al. 2010, A\&A, 518, L2

Polychroni, D., Schisano, E., Elia, D., et al. 2013, ApJ, 777, L33

Reach, W. T., Dwek, E., Fixsen, D. J., et al. 1995, ApJ, 451, 188

Reid, M. J., Menten, K. M., Brunthaler, A., et al. 2014, ApJ, 783, 130

Riener, M., Faesi, C. M., Forbrich, J., \& Lada, C. J. 2018, A\&A, 612, A81

Robitaille, T., Rice, T., Beaumont, C., et al. 2019, Astrodendro: Astronomical data dendrogram creator

Rumble, D., Hatchell, J., Gutermuth, R. A., et al. 2015, MNRAS, 448, 1551

Russeil, D., Pestalozzi, M., Mottram, J. C., et al. 2011, A\&A, 526, A151

Sadavoy, S. I., di Francesco, J., André, P., et al. 2012, A\&A, 540, A10

Sadavoy, S. I., Di Francesco, J., Johnstone, D., et al. 2013, ApJ, 767, 126

Sanhueza, P., Jackson, J. M., Foster, J. B., et al. 2013, ApJ, 773, 123

Sanhueza, P., Jackson, J. M., Zhang, Q., et al. 2017, ApJ, 841, 97

Sanhueza, P., Contreras, Y., Wu, B., et al. 2019, ApJ, 886, 102

Schmidt, M. 1959, ApJ, 129, 243

Shetty, R., Kauffmann, J., Schnee, S., \& Goodman, A. A. 2009a, ApJ, 696, 676

Shetty, R., Kauffmann, J., Schnee, S., Goodman, A. A., \& Ercolano, B. 2009b, ApJ, 696, 2234

Skrutskie, M. F., Cutri, R. M., Stiening, R., et al. 2006, AJ, 131, 1163

Soam, A., Liu, T., Andersson, B. G., et al. 2019, ApJ, 883, 95

Stark, A. A. 1984, ApJ, 281, 624

Stutzki, J. 2014, Astrophysics Source Code Library [record ascl:1406.018]

Stutzki, J., \& Guesten, R. 1990, ApJ, 356, 513

Tabatabaei, F. S., Braine, J., Xilouris, E. M., et al. 2014, A\&A, 561, A95

Tang, M., Liu, T., Qin, S.-L., et al. 2018, ApJ, 856, 141

Tang, Y.-W., Koch, P. M., Peretto, N., et al. 2019, ApJ, 878, 10

Tasker, E. J., Wadsley, J., \& Pudritz, R. 2015, ApJ, 801, 33

Tatematsu, K., Liu, T., Ohashi, S., et al. 2017, ApJS, 228, 12

Tatematsu, K., Liu, T., Kim, G., et al. 2020, ApJ, 895, 119

Teixeira, P. S., Takahashi, S., Zapata, L. A., \& Ho, P. T. P. 2016, A\&A, 587, A47

Thompson, M. A., Urquhart, J. S., Moore, T. J. T., \& Morgan, L. K. 2012, MNRAS, 421, 408

Tosaki, T., Kohno, K., Harada, N., et al. 2017, PASJ, 69, 18

Traficante, A., Fuller, G. A., Peretto, N., Pineda, J. E., \& Molinari, S. 2015, MNRAS, 451, 3089

Traficante, A., Fuller, G. A., Billot, N., et al. 2017, MNRAS, 470, 3882

Traficante, A., Fuller, G. A., Duarte-Cabral, A., et al. 2020, MNRAS, 491, 4310
Trujillo-Gomez, S., Reina-Campos, M., \& Kruijssen, J. M. D. 2019, MNRAS, 488, 3972

Urquhart, J. S., König, C., Giannetti, A., et al. 2018, MNRAS, 473, 1059

Valtchanov, I. 2017, The Spectral and Photometric Imaging REceiver (SPIRE) handbook, 3rd edn. (Herschel Space Observatory)

Wang, K., Zhang, Q., Testi, L., et al. 2014, MNRAS, 439, 3275

Wang, K., Testi, L., Ginsburg, A., et al. 2015, MNRAS, 450, 4043

Wang, K., Zahorecz, S., Cunningham, M. R., et al. 2018, Res. Notes Am. Astron. Soc., 2, 2

Ward-Thompson, D., Di Francesco, J., Hatchell, J., et al. 2007, PASP, 119, 855

Whitaker, J. S., Jackson, J. M., Rathborne, J. M., et al. 2017, AJ, 154, 140

Williams, J. P., de Geus, E. J., \& Blitz, L. 1994, ApJ, 428, 693

Wright, E. L., Eisenhardt, P. R. M., Mainzer, A. K., et al. 2010, AJ, 140, 1868

Yamamura, I., Makiuti, S., Ikeda, N., et al. 2010, AKARI/FIS All-Sky Survey Bright Source Catalogue Version 1.0 http://www.ir.isas.jaxa.jp/ AKARI/Archive/Catalogues/PSC/RN/AKARI-FIS_BSC_V1_RN.pdf

Yi, H.-W., Lee, J.-E., Liu, T., et al. 2018, ApJS, 236, 51

Zahorecz, S., Jimenez-Serra, I., Wang, K., et al. 2016, A\&A, 591, A105

Zhang, Q., Wang, K., Lu, X., \& Jiménez-Serra, I. 2015, ApJ, 804, 141

Zhang, G.-Y., Xu, J.-L., Vasyunin, A. I., et al. 2018, A\&A, 620, A163

Zucker, C., Battersby, C., \& Goodman, A. 2018, ApJ, 864, 153

${ }^{1}$ Department of Physics, PO box 64, 00014 University of Helsinki, Finland e-mail: emma .mannfors@helsinki . fi

2 Astronomy Department, Universidad de Chile, Camino el Observatorio 1515, Las Condes, Santiago de Chile, Chile

3 Astrophysics Research Institute, Liverpool John Moores University, IC2, Liverpool Science Park, 146 Brownlow Hill, Liverpool L3 5RF, UK

4 Yunnan Observatories, Chinese Academy of Sciences, Phoenix Mountain, East Suburb of Kunming, 650216 Yunnan, PR China

5 Chinese Academy of Sciences South America Center for Astronomy, National Astronomical Observatories, CAS, Beijing 100101, PR China

6 Nobeyama Radio Observatory, National Astronomical Observatory of Japan, National Institutes of Natural Sciences, Nobeyama, Minamimaki, Minamisaku, Nagano 384-1305, Japan

7 Korea Astronomy and Space Science Institute, 776 Daedeokdae-ro, Yuseong-gu, Daejon 34055, Republic of Korea

8 University of Science \& Technology, 176 Gajeong-dong, Yuseonggu, Daejeon, Republic of Korea

9 Shanghai Astronomical Observatory, Chinese Academy of Sciences, 80 Nandan Road, Shanghai 200030, PR China

10 Institut UTINAM - UMR 6213 - CNRS - Univ. Bourgogne Franche Comté, OSU THETA, 41bis avenue de l'Observatoire, 25000 Besançon, France

11 East Asian Observatory, 660 N. A'ohōkū Place, University Park, Hilo, HI 96720, USA

12 National Astronomical Observatory of Japan, National Institutes of Natural Sciences, 2-21-1 Osawa, Mitaka, Tokyo 181-8588, Japan

13 Department of Astronomical Science, The Graduate University for Advanced Studies, SOKENDAI, 2-21-1 Osawa, Mitaka, Tokyo 181-8588, Japan

14 Institute of Astronomy and Astrophysics, Academia Sinica. 11F of Astronomy-Mathematics Building, AS/NTU No.1, Section 4, Roosevelt Rd, Taipei 10617, Taiwan

15 SOFIA Science Center, Universities Space Research Association, NASA Ames Research Center, Moffett Field, CA 94035, USA

16 IAPS-INAF, via Fosso del Cavaliere 100, 00133 Rome, Italy 


\section{Appendix A: Instrument specifications}

In the following tables we present properties of the instruments used in our study. Table A. 1 lists the parameters of the continuum observations and Table A.2 the parameters of the line observations.

Table A.1. Continuum instrumental characteristics.

\begin{tabular}{lccc}
\hline \hline Band & $\begin{array}{c}\text { Central } \\
\text { Wavelength } \\
(\mu \mathrm{m})\end{array}$ & $\begin{array}{c}\text { Beam } \\
\text { FWHM } \\
\left({ }^{\prime \prime}\right)\end{array}$ & $\begin{array}{c}\text { Pixel } \\
\text { size } \\
\left({ }^{\prime \prime}\right)\end{array}$ \\
\hline PACS blue $^{(a)}$ & 70 & 5.6 & 3.2 \\
PACS green $^{(a)}$ & 100 & 6.8 & 3.2 \\
PACS red $^{(a)}$ & 160 & 12.0 & 3.2 \\
SPIRE PSW $^{b}$ & 250 & 17.9 & 6.0 \\
SPIRE PMW $^{(b)}$ & 350 & 24.2 & 10.0 \\
SPIRE PLW $^{(b)}$ & 500 & 35.4 & 14.0 \\
SCUBA-2 $^{(c, d)}$ & $850^{(c)}$ & $14.6^{(d)}$ & $4.0^{(c)}$ \\
\hline
\end{tabular}

Notes. Central wavelength, beam FWHM, and pixel size of the 7 bands used in this paper. ${ }^{(a)}$ Sources from Exter (2017). ${ }^{(b)}$ Sources from Valtchanov (2017). ${ }^{(c)}$ Sources from Holland et al. (2013). ${ }^{(d)}$ Sources from Dempsey et al. (2013).
Table A.2. TRAO molecular line instrumental characteristics.

\begin{tabular}{lccccc}
\hline \hline $\begin{array}{l}\text { Band } \\
\mathrm{J}=(1-0)\end{array}$ & $\begin{array}{c}\text { Main-beam } \\
\text { Efficiency } \eta_{\mathrm{B}} \\
(\%)\end{array}$ & $\begin{array}{c}\text { Beam } \\
\text { FWHM } \\
\left({ }^{\prime \prime}\right)\end{array}$ & $\begin{array}{c}\text { Pixel } \\
\text { size } \\
\left({ }^{\prime \prime}\right)\end{array}$ & $\begin{array}{c}\text { System } \\
\text { Temperature } \\
(\mathrm{K})\end{array}$ & $\begin{array}{c}\text { Typical } \\
\text { Sensitivity } \\
\left(T_{\mathrm{A}}^{*}\right)\end{array}$ \\
\hline${ }^{12} \mathrm{CO}$ & 54 & 45 & 24.0 & 500 & $0.5^{(a)}$ \\
${ }^{13} \mathrm{CO}$ & 51 & 47 & 24.0 & 250 & $0.2^{(a)}$ \\
\hline
\end{tabular}

Notes. Instrumental parameters for the ${ }^{12} \mathrm{CO}$ and ${ }^{13} \mathrm{CO}$ TRAO observations. ${ }^{(a)}$ Typical sensitivity is calculated at a spectral resolution of $0.33 \mathrm{~km} \mathrm{~s}^{-1}$ over the mapping field for the typical 40 minute integration time (Liu et al. 2018a). 


\section{Appendix B: SED fits using PACS $160 \mu \mathrm{m}$ and SCUBA-2 $850 \mu \mathrm{m}$ data}

In the main paper we relied mainly on SED fits to $250-500 \mu \mathrm{m}$ observations because we do not have $160 \mu \mathrm{m}$ data for all fields and the inclusion of the shorter wavelength might bias the column density estimates. In Sect. 4.4 we also studied the hypothesis that the flattening of the dust emission spectrum at long wavelengths could be visible already in the $850 \mu \mathrm{m}$ observations. Also in this case we used fits to the $250-500 \mu \mathrm{m}$ range, comparing the $850 \mu \mathrm{m}$ flux densities to the MBB SEDs fitted to the shorter wavelength observations. The flux calculation in this analysis differs slightly from that used in the main paper, as here we subtract the background value in an annulus around the clump from the flux of the clump. As a result of this background subtraction, MBB fits are also performed on a clump-byclump basis instead of on the field as a whole. The background is removed from the clump in an annulus of width $0.5^{\prime}, 20^{\prime \prime}$ from the edge of the clump.

For completeness, we show in Fig. B.1 SED fits for all wavelength combinations $250-500 \mu \mathrm{m}, 160-500 \mu \mathrm{m}, 250-850 \mu \mathrm{m}$, and
$160-850 \mu \mathrm{m}$. We also derive temperatures, column densities, and masses for clumps with $250-850 \mu \mathrm{m}$ data using the MBB SED fit described in Section 3.2 with a variable opacity spectral index (Table B.1). The mass is calculated as in Eq. (7). Owing to SCUBA-2 filtering, we remove the background on all data analyzed. This results in slightly different predicted quantities in the $160-500 \mu \mathrm{m}$ and $250-50 \mu \mathrm{m}$ bands than we derive in the main paper.

Compared to the SPIRE-only fits, the inclusion of the $160 \mu \mathrm{m}$ point increases the temperature estimates by an average of $0.5 \mathrm{~K}$, whether the $850 \mu \mathrm{m}$ data point is included in the fit or not. For dust temperatures close to $10 \mathrm{~K}$, this corresponds to more than a factor of 2 decrease in the mass estimates, thus indicating a potentially significant bias. In contrast, the inclusion of the $850 \mu \mathrm{m}$ point decreases temperatures by close to $1 \mathrm{~K}$, thereby leading to significantly higher derived column densities. Mass derivation is not strongly affected by the inclusion of $850 \mu \mathrm{m}$ data. We find an increase in opacity spectral index with the inclusion of the $850 \mu \mathrm{m}$ data point, both with and without $160 \mu \mathrm{m}$ data.

Table B.1. SED fits with all wavelength combinations.

\begin{tabular}{|c|c|c|c|c|c|c|c|c|}
\hline $\begin{array}{l}\text { Band }(\mu \mathrm{m}) \\
\text { Clump } \\
\text { type }\end{array}$ & \multicolumn{4}{|c|}{$\begin{array}{r}\langle T\rangle \\
(\mathrm{K})\end{array}$} & \multicolumn{4}{|c|}{$\begin{array}{c}\left\langle N\left(\mathrm{H}_{2}\right)\right\rangle \\
\left(10^{21} \mathrm{~cm}^{-2}\right)\end{array}$} \\
\hline All & $15.5 \pm 5.3$ & $16.1 \pm 6.3$ & $14.3 \pm 6.4$ & $14.9 \pm 5.3$ & $18.3 \pm 56.8$ & $15.4 \pm 31.4$ & $21.5 \pm 30.3$ & $20.5 \pm 57.4$ \\
\hline PS-C & $15.9 \pm 1.3$ & $16.2 \pm 0.3$ & $12.7 \pm 0.2$ & $14.2 \pm 0.6$ & $6.3 \pm 2.4$ & $8.2 \pm 5.4$ & $19.4 \pm 13.1$ & $10.2 \pm 5.1$ \\
\hline PS & $14.8 \pm 3.6$ & $17.1 \pm 7.1$ & $16.0 \pm 7.7$ & $13.8 \pm 3.9$ & $7.4 \pm 3.0$ & $6.8 \pm 4.3$ & $16.5 \pm 24.7$ & $16.2 \pm 15.7$ \\
\hline PRE & $16.1 \pm 5.5$ & $16.5 \pm 6.4$ & $15.1 \pm 6.7$ & $15.6 \pm 5.5$ & $20.9 \pm 66.0$ & $17.0 \pm 36.1$ & $22.2 \pm 33.7$ & $20.7 \pm 62.7$ \\
\hline SL & $14.0 \pm 4.8$ & $14.6 \pm 5.7$ & $11.9 \pm 4.4$ & $12.8 \pm 4.5$ & $12.6 \pm 18.4$ & $12.4 \pm 14.4$ & $20.3 \pm 18.5$ & $20.9 \pm 42.7$ \\
\hline SL PRE & $15.6 \pm 5.4$ & $16.0 \pm 6.3$ & $14.3 \pm 6.3$ & $14.9 \pm 5.4$ & $18.7 \pm 57.6$ & $15.9 \pm 32.2$ & $21.8 \pm 30.6$ & $20.7 \pm 58.3$ \\
\hline PS PS-C & $15.1 \pm 3.2$ & $17.0 \pm 6.6$ & $15.6 \pm 7.3$ & $13.9 \pm 3.3$ & $7.1 \pm 2.9$ & $7.0 \pm 4.5$ & $16.9 \pm 23.6$ & $14.5 \pm 13.8$ \\
\hline Band $(\mu \mathrm{m})$ & $(160-500)$ & $(250-500)$ & $(250-850)$ & $(160-850)$ & $(160-500)$ & $(250-500)$ & $(250-850)$ & $(160-850)$ \\
\hline $\begin{array}{l}\text { Clump } \\
\text { type }\end{array}$ & \multicolumn{4}{|c|}{$\begin{array}{l}\langle M\rangle \\
\left(\mathrm{M}_{\odot}\right)\end{array}$} & \multicolumn{4}{|c|}{$\beta$} \\
\hline All & $154 \pm 276$ & $137 \pm 258$ & $149 \pm 262$ & $139 \pm 249$ & $1.88 \pm 0.66$ & $1.86 \pm 0.63$ & $2.2 \pm 0.66$ & $2.03 \pm 0.62$ \\
\hline PS-C & $10 \pm 2$ & $10 \pm 3$ & $16 \pm 5$ & $12 \pm 3$ & $1.44 \pm 0.07$ & $1.45 \pm 0.09$ & $2.03 \pm 0.17$ & $1.77 \pm 0.03$ \\
\hline PS & $14 \pm 9$ & $31 \pm 40$ & $34 \pm 38$ & $45 \pm 68$ & $1.5 \pm 0.47$ & $1.74 \pm 0.32$ & $2.04 \pm 0.85$ & $1.84 \pm 0.55$ \\
\hline PRE & $209 \pm 309$ & $175 \pm 287$ & $191 \pm 290$ & $176 \pm 275$ & $1.95 \pm 0.68$ & $1.92 \pm 0.67$ & $2.18 \pm 0.68$ & $2.03 \pm 0.65$ \\
\hline SL & $18 \pm 29$ & $17 \pm 31$ & $20 \pm 31$ & $18 \pm 30$ & $1.73 \pm 0.59$ & $1.7 \pm 0.54$ & $2.29 \pm 0.52$ & $2.07 \pm 0.54$ \\
\hline SL PRE & $159 \pm 279$ & $143 \pm 264$ & $156 \pm 268$ & $144 \pm 254$ & $1.89 \pm 0.66$ & $1.87 \pm 0.64$ & $2.21 \pm 0.65$ & $2.04 \pm 0.62$ \\
\hline PS PS-C & $13 \pm 8$ & $29 \pm 38$ & $31 \pm 36$ & $41 \pm 65$ & $1.49 \pm 0.40$ & $1.7 \pm 0.31$ & $2.04 \pm 0.79$ & $1.82 \pm 0.47$ \\
\hline
\end{tabular}

Notes. Results of the MBB fit to different wavelength ranges, with opacity spectral index as a free parameter. Temperature and column density are the average value over the clump footprint. To ensure consistency between SPIRE, PACS, and SCUBA-2 data, the background has been subtracted from all fields. The errors are the standard deviation of the distribution. This table includes only those fields for which distances (to calculate mass) are available. 


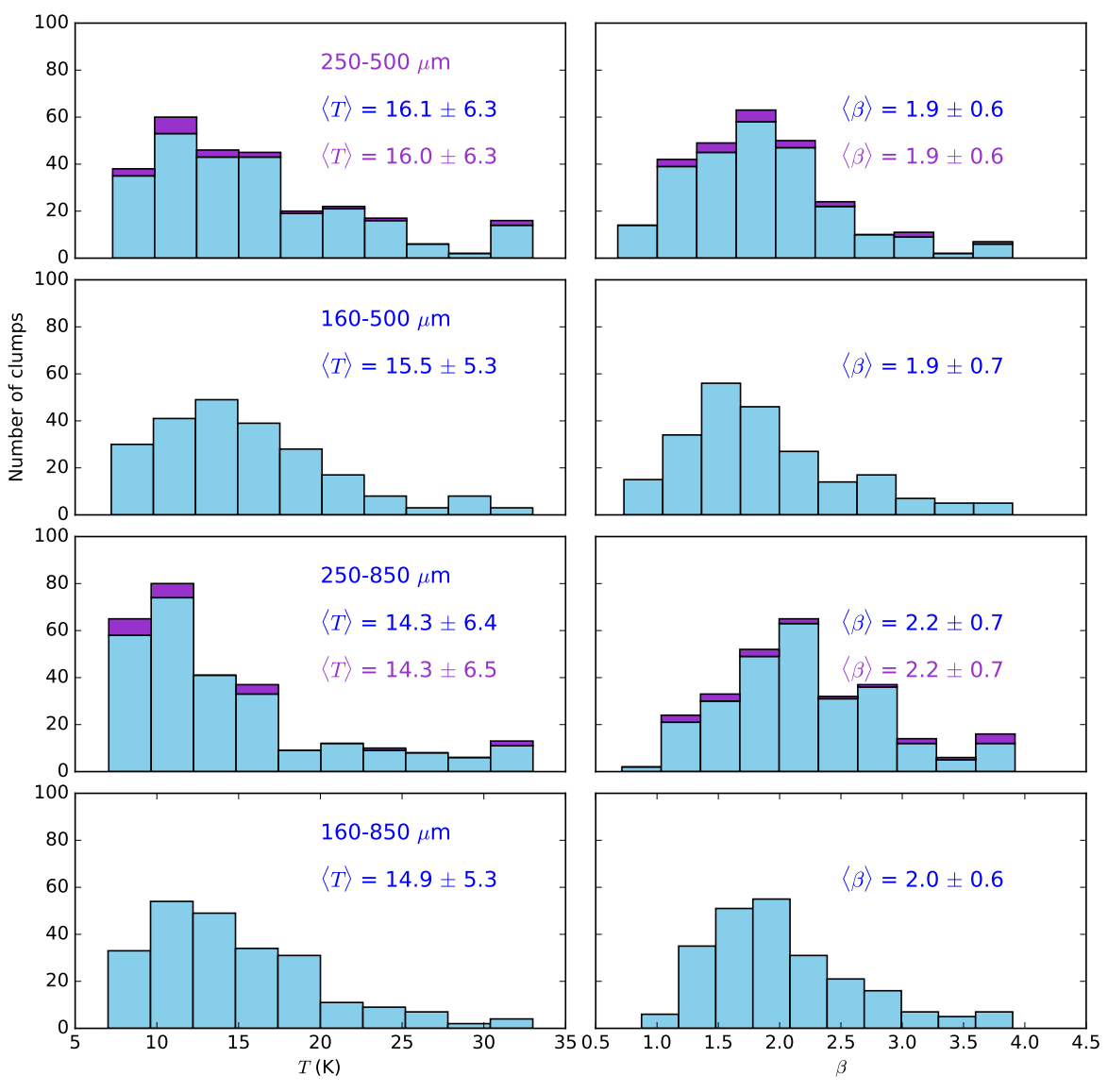

Fig. B.1. Comparison of MBB fits of clump spectra performed using different band combinations. The purple histograms correspond to all the clumps and the blue histograms to the subsets of clumps for which $160 \mu \mathrm{m}$ measurements also exist. The mean values and standard deviations of the temperature and the dust opacity spectral index distributions are given in the frames, written with the same colors. 


\section{Appendix C: Data coverage}

In Table C.1 we present what data we have for each field. In the case of Herschel, this also includes fields that are only partially covered by observations. The table also lists the YSO catalogs used.

Table C.1. Coverage of each SCUBA-2 field.

\begin{tabular}{|c|c|c|c|c|c|c|c|c|c|c|c|}
\hline \multirow{3}{*}{$\begin{array}{l}\text { SCUBA-2 } \\
\text { Field }\end{array}$} & \multirow{2}{*}{\multicolumn{2}{|c|}{$\begin{array}{l}\text { Data } \\
\text { Herschel }\end{array}$}} & \multirow{3}{*}{ TRAO } & \multicolumn{6}{|c|}{ YSO catalog } & \multirow{2}{*}{\multicolumn{2}{|c|}{ Herschel }} \\
\hline & & & & & zer & $\mathrm{Ma}$ & & & ARI & & \\
\hline & $160 \mu \mathrm{m}$ & SPIRE & & type I & type II & type I/II & type III & FIS & PSC & $70 \mu \mathrm{m}$ & $100 \mu \mathrm{m}$ \\
\hline $\mathrm{G} 001.36+20.96$ & $\mathrm{X}$ & $\mathrm{X}$ & & & & $\mathrm{X}$ & & $\mathrm{X}$ & $\mathrm{X}$ & $\mathrm{X}$ & \\
\hline G005.93-01.00 & $\mathrm{X}$ & $\mathrm{X}$ & $\mathrm{X}$ & & & $\mathrm{X}$ & & $\mathrm{X}$ & $\mathrm{X}$ & & \\
\hline G006.01+36.74 & $\mathrm{X}$ & $\mathrm{X}$ & & & & & & $\mathrm{X}$ & $\mathrm{X}$ & & \\
\hline $\mathrm{G} 010.21+02.40$ & $\mathrm{X}$ & $\mathrm{X}$ & $\mathrm{X}$ & & & $\mathrm{X}$ & $\mathrm{X}$ & $\mathrm{X}$ & $\mathrm{X}$ & & $\mathrm{X}$ \\
\hline G014.14-00.55 & $\mathrm{X}$ & $\mathrm{X}^{(a)}$ & $\mathrm{X}$ & $\mathrm{X}$ & $\mathrm{X}$ & $\mathrm{X}$ & & $\mathrm{X}$ & $\mathrm{X}$ & $\mathrm{X}$ & \\
\hline G014.15-00.55 & $\mathrm{X}$ & $\mathrm{X}^{(a)}$ & $\mathrm{X}$ & $\mathrm{X}$ & $\mathrm{X}$ & $\mathrm{X}$ & & $\mathrm{X}$ & $\mathrm{X}$ & $X$ & \\
\hline G014.22-00.19 & $\mathrm{X}$ & $\mathrm{X}$ & & & $\mathrm{X}$ & $\mathrm{X}$ & & $\mathrm{X}$ & $\mathrm{X}$ & $\mathrm{X}$ & \\
\hline G014.72-00.19 & $\mathrm{X}$ & $\mathrm{X}$ & & & $\mathrm{X}$ & $\mathrm{X}$ & & $\mathrm{X}$ & $\mathrm{X}$ & $\mathrm{X}$ & \\
\hline G014.73-00.19 & $\mathrm{X}$ & $\mathrm{X}$ & & & $\mathrm{X}$ & $\mathrm{X}$ & & $\mathrm{X}$ & $\mathrm{X}$ & $\mathrm{X}$ & \\
\hline G016.28-00.45 & $\mathrm{X}$ & $\mathrm{X}$ & & $\mathrm{X}$ & $\mathrm{X}$ & $\mathrm{X}$ & $\mathrm{X}$ & $\mathrm{X}$ & $\mathrm{X}$ & $\mathrm{X}$ & \\
\hline G016.37-00.61 & $\mathrm{X}$ & $\mathrm{X}$ & $\mathrm{X}$ & & & $\mathrm{X}$ & & $\mathrm{X}$ & $\mathrm{X}$ & $\mathrm{X}$ & \\
\hline G016.43-00.62 & $\mathrm{X}$ & $\mathrm{X}$ & $\mathrm{X}$ & $\mathrm{X}$ & $\mathrm{X}$ & $\mathrm{X}$ & & $\mathrm{X}$ & $\mathrm{X}$ & $\mathrm{X}$ & \\
\hline G016.97+00.28 & $\mathrm{X}$ & $\mathrm{X}$ & $\mathrm{X}$ & & $\mathrm{X}$ & $\mathrm{X}$ & & $\mathrm{X}$ & $\mathrm{X}$ & $\mathrm{X}$ & \\
\hline G017.23-01.47 & & $\mathrm{X}$ & $\mathrm{X}$ & & & $\mathrm{X}$ & & $\mathrm{X}$ & $\mathrm{X}$ & & \\
\hline $\mathrm{G} 017.38+02.26$ & & & & & & $\mathrm{X}$ & & $\mathrm{X}$ & $\mathrm{X}$ & & \\
\hline G023.35-00.26 & $\mathrm{X}$ & $\mathrm{X}$ & & & $\mathrm{X}$ & $\mathrm{X}$ & & $\mathrm{X}$ & $\mathrm{X}$ & $\mathrm{X}$ & \\
\hline G023.68+00.58 & $\mathrm{X}$ & $\mathrm{X}$ & $\mathrm{X}$ & & $\mathrm{X}$ & $\mathrm{X}$ & & $\mathrm{X}$ & $\mathrm{X}$ & $\mathrm{X}$ & \\
\hline $\mathrm{G} 023.69+00.58$ & $\mathrm{X}$ & $\mathrm{X}$ & $\mathrm{X}$ & & $\mathrm{X}$ & $\mathrm{X}$ & & $\mathrm{X}$ & $\mathrm{X}$ & $\mathrm{X}$ & \\
\hline G024.04+00.27 & $\mathrm{X}$ & $\mathrm{X}$ & $\mathrm{X}$ & & $\mathrm{X}$ & $\mathrm{X}$ & & $\mathrm{X}$ & $\mathrm{X}$ & $\mathrm{X}$ & \\
\hline G026.54+00.72 & $\mathrm{X}$ & $\mathrm{X}$ & $\mathrm{X}$ & & & $\mathrm{X}$ & & $\mathrm{X}$ & $\mathrm{X}$ & $\mathrm{X}$ & \\
\hline G033.73-00.01 & $\mathrm{X}$ & $\mathrm{X}$ & $\mathrm{X}$ & $\mathrm{X}$ & $\mathrm{X}$ & $\mathrm{X}$ & $\mathrm{X}$ & $\mathrm{X}$ & $\mathrm{X}$ & $\mathrm{X}$ & \\
\hline G034.74-01.39 & $\mathrm{X}$ & $\mathrm{X}$ & $\mathrm{X}$ & & & $\mathrm{X}$ & & $\mathrm{X}$ & $\mathrm{X}$ & $\mathrm{X}$ & \\
\hline G035.38-01.77 & $\mathrm{X}$ & $\mathrm{X}^{(a)}$ & $\mathrm{X}$ & & & $\mathrm{X}$ & & $\mathrm{X}$ & $\mathrm{X}$ & $\mathrm{X}$ & \\
\hline G035.50-00.31 & $\mathrm{X}$ & $\mathrm{X}$ & & & $\mathrm{X}$ & $\mathrm{X}$ & & $\mathrm{X}$ & $\mathrm{X}$ & $\mathrm{X}$ & \\
\hline G037.93+02.18 & $\mathrm{X}$ & $\mathrm{X}$ & & & & $\mathrm{X}$ & $\mathrm{X}$ & $\mathrm{X}$ & $\mathrm{X}$ & & $\mathrm{X}$ \\
\hline G039.74+01.98 & $\mathrm{X}$ & $\mathrm{X}$ & $\mathrm{X}$ & & & $\mathrm{X}$ & $\mathrm{X}$ & $\mathrm{X}$ & $\mathrm{X}$ & & \\
\hline $\mathrm{G} 057.12+03.63$ & & & & & & $\mathrm{X}$ & & $\mathrm{X}$ & $\mathrm{X}$ & & \\
\hline G069.81-01.67 & $\mathrm{X}$ & $\mathrm{X}$ & & & & $\mathrm{X}$ & & $\mathrm{X}$ & $\mathrm{X}$ & & $\mathrm{X}$ \\
\hline G070.40-01.40 & $\mathrm{X}$ & $\mathrm{X}$ & & & & $\mathrm{X}$ & & $\mathrm{X}$ & $\mathrm{X}$ & & $\mathrm{X}$ \\
\hline G074.13+00.11 & $\mathrm{X}$ & $\mathrm{X}$ & & & & $\mathrm{X}$ & & $\mathrm{X}$ & $\mathrm{X}$ & $\mathrm{X}$ & \\
\hline G082.40-01.84 & $\mathrm{X}$ & $\mathrm{X}$ & $\mathrm{X}$ & & & & $\mathrm{X}$ & $\mathrm{X}$ & $\mathrm{X}$ & & $\mathrm{X}$ \\
\hline G082.42-01.84 & $\mathrm{X}$ & $\mathrm{X}$ & $\mathrm{X}$ & & & & $\mathrm{X}$ & $\mathrm{X}$ & $\mathrm{X}$ & & $\mathrm{X}$ \\
\hline G087.07-04.20 & $\mathrm{X}$ & $\mathrm{X}$ & & & & $\mathrm{X}$ & & $\mathrm{X}$ & $\mathrm{X}$ & & \\
\hline G089.66-06.62 & $\mathrm{X}$ & $\mathrm{X}$ & & & & $\mathrm{X}$ & & $\mathrm{X}$ & $\mathrm{X}$ & & $\mathrm{X}$ \\
\hline $\mathrm{G} 091.88+04.17$ & & $\mathrm{X}$ & & & & $\mathrm{X}$ & & $\mathrm{X}$ & $\mathrm{X}$ & & \\
\hline G092.04+03.92 & $\mathrm{X}$ & $\mathrm{X}$ & & & & $\mathrm{X}$ & & $\mathrm{X}$ & $\mathrm{X}$ & & $\mathrm{X}$ \\
\hline G092.28+03.79 & $\mathrm{X}$ & $\mathrm{X}$ & & & & $\mathrm{X}$ & & $\mathrm{X}$ & $\mathrm{X}$ & & $\mathrm{X}$ \\
\hline G093.51-04.31 & $\mathrm{X}$ & $\mathrm{X}$ & & & & $\mathrm{X}$ & & $\mathrm{X}$ & $\mathrm{X}$ & $\mathrm{X}$ & $\mathrm{X}$ \\
\hline $\mathrm{G} 105.44+09.88$ & & $\mathrm{X}$ & $\mathrm{X}$ & & & $\mathrm{X}$ & & $\mathrm{X}$ & $\mathrm{X}$ & & \\
\hline G107.18+05.44 & $\mathrm{X}$ & $\mathrm{X}$ & $\mathrm{X}$ & & & $\mathrm{X}$ & & $\mathrm{X}$ & $\mathrm{X}$ & & $\mathrm{X}$ \\
\hline G107.26+05.71 & $\mathrm{X}$ & $\mathrm{X}$ & & & & $\mathrm{X}$ & & $\mathrm{X}$ & $\mathrm{X}$ & & \\
\hline $\mathrm{G} 109.70+02.52$ & $\mathrm{X}$ & $\mathrm{X}$ & & & & $\mathrm{X}$ & & $\mathrm{X}$ & $\mathrm{X}$ & $\mathrm{X}$ & \\
\hline $\mathrm{G} 109.81+02.72$ & $\mathrm{X}$ & $\mathrm{X}$ & $\mathrm{X}$ & & & $\mathrm{X}$ & & $\mathrm{X}$ & $\mathrm{X}$ & & \\
\hline G139.14-03.23 & $\mathrm{X}$ & $\mathrm{X}$ & $\mathrm{X}$ & & & $\mathrm{X}$ & & $\mathrm{X}$ & $\mathrm{X}$ & & $\mathrm{X}$ \\
\hline G159.23-20.09 & $\mathrm{X}$ & $\mathrm{X}$ & $\mathrm{X}$ & & & $\mathrm{X}$ & & $\mathrm{X}$ & $\mathrm{X}$ & $\mathrm{X}$ & $\mathrm{X}$ \\
\hline G162.46-08.69 & $\mathrm{X}$ & $\mathrm{X}$ & & & & $\mathrm{X}$ & & $\mathrm{X}$ & $\mathrm{X}$ & $\mathrm{X}$ & \\
\hline G171.53-14.91 & $\mathrm{X}$ & $\mathrm{X}$ & & & & $\mathrm{X}$ & & $\mathrm{X}$ & $\mathrm{X}$ & $X$ & $\mathrm{X}$ \\
\hline $\mathrm{G} 172.89+02.28$ & & & & & & $\mathrm{X}$ & & $\mathrm{X}$ & $\mathrm{X}$ & & \\
\hline $\mathrm{G} 173.15+02.40$ & $\mathrm{X}$ & & $\mathrm{X}$ & & & $\mathrm{X}$ & & $\mathrm{X}$ & $\mathrm{X}$ & $\mathrm{X}$ & $\mathrm{X}$ \\
\hline G178.28-00.60 & $\mathrm{X}$ & $\mathrm{X}$ & & & & $\mathrm{X}$ & & $\mathrm{X}$ & $\mathrm{X}$ & $\mathrm{X}$ & \\
\hline G195.73-02.28 & $\mathrm{X}$ & $\mathrm{X}$ & $\mathrm{X}$ & & & $\mathrm{X}$ & & $\mathrm{X}$ & $\mathrm{X}$ & & $\mathrm{X}$ \\
\hline G202.31+02.53 & $\mathrm{X}$ & $X$ & $\mathrm{X}$ & & & & & $\mathrm{X}$ & $\mathrm{X}$ & & $\mathrm{X}$ \\
\hline G215.87-17.50 & & & $\mathrm{X}$ & & & $\mathrm{X}$ & & $\mathrm{X}$ & $\mathrm{X}$ & & \\
\hline
\end{tabular}

Notes. ' $\mathrm{X}$ ' denotes fields covered by Herschel or TRAO data, or the YSO catalog. Spitzer and Marton types I-III refer to YSO classifications.

${ }^{(a)}$ This field is located at the edge of the Herschel map; the available data are not sufficient to calculate column densities and temperatures. 


\section{Appendix D: Distances to sources}

The following tables list the distance estimates used in this study. Table D.1 shows new kinematic distances derived for sources with ${ }^{12} \mathrm{CO}$ TRAO data, as well as the galactocentric and both near- and far heliocentric distances. Field G105.44+09.88 uses ${ }^{12} \mathrm{CO}$ line data from Wang et al. (2018). The distances are derived as explained in Sect. 3.1. Table D.2 summarizes all distance estimates, including the values from Montillaud et al. (2015) and Planck Collaboration XXVIII (2016).

Table D.1. Input values and results of kinematic distance calculations.

\begin{tabular}{lccccc}
\hline \hline Field & $\begin{array}{c}{ }^{2} \mathrm{CO} \\
v_{L S R} \\
\left(\mathrm{~km} \mathrm{~s}^{-1}\right)\end{array}$ & $\begin{array}{c}R \\
(\mathrm{kpc})\end{array}$ & $\begin{array}{c}d \\
(\mathrm{kpc})\end{array}$ & $\begin{array}{c}d \\
(\mathrm{kpc})\end{array}$ & $\begin{array}{c}\text { Adopted } \\
\text { distance } \\
(\mathrm{kpc})\end{array}$ \\
\hline G005.91-01.00 & 14.341 & 5.227 & 3.301 & 13.608 & 3.3 \\
G010.20+2.39 & 5.582 & 7.53 & 0.988 & 15.744 & 1.0 \\
G014.14-0.55 & 20.403 & 6.214 & 2.386 & 14.099 & 2.4 \\
G016.36-0.62 & 22.111 & 6.321 & 2.306 & 14.006 & 2.3 \\
G016.42-0.63 & 22.483 & 6.299 & 2.331 & 13.976 & 2.3 \\
G016.96+0.27 & 23.609 & 6.269 & 2.373 & 13.888 & 2.4 \\
G017.21-01.46 & 42.355 & 5.17 & 3.602 & 12.637 & 3.6 \\
G023.68+0.57 & 9.068 & 7.813 & 0.756 & 14.812 & 0.8 \\
G024.04+0.26 & 7.988 & 7.912 & 0.649 & 14.877 & 0.6 \\
G026.53+0.71 & 8.174 & 7.956 & 0.613 & 14.597 & 0.6 \\
G033.72-0.027 & 10.887 & 7.912 & 0.719 & 13.421 & 0.7 \\
G034.73-1.39 & 14.149 & 7.74 & 0.948 & 13.023 & 0.9 \\
G035.37-01.76 & 42.86 & 6.419 & 2.809 & 11.054 & 2.8 \\
G035.49-0.31 & 13.238 & 7.806 & 0.873 & 12.969 & 0.9 \\
G039.38-1.98 & 31.112 & 7.03 & 2.061 & 11.079 & 2.1 \\
G082.39-1.84 & -3.231 & 8.763 & -1.284 & 3.535 & 3.5 \\
G105.44+09.88 $(a)$ & -10.48 & 9.087 & -6.188 & 1.669 & 1.7 \\
G107.17+5.44 & -9.502 & 9.047 & -6.495 & 1.477 & 1.5 \\
G109.79+2.71 & -9.805 & 9.067 & -7.15 & 1.394 & 1.4 \\
G139.19-3.29 & -31.792 & 11.121 & -16.068 & 3.201 & 3.2 \\
G159.23-34.51 & -4.927 & 9.223 & -16.664 & 0.769 & 0.8 \\
G173.15+02.38 & -17.722 & 26.556 & -34.976 & 18.098 & 18.1 \\
G195.74-2.29 & 4.548 & 9.355 & -17.248 & 0.885 & 0.9 \\
G202.32+02.51 & 6.825 & 9.412 & -16.704 & 0.978 & 1.0 \\
G215.8-17.51 & 10.082 & 9.377 & -14.845 & 1.056 & 1.1 \\
\hline & & & & &
\end{tabular}

Notes. Velocities have been found from TRAO CO line data. ${ }^{(a)}$ Velocity estimate derived using the ${ }^{12}$ line measured by Wang et al. (2018).
Table D.2. Distances of the fields.

\begin{tabular}{|c|c|c|c|}
\hline $\begin{array}{l}\text { SCUBA-2 } \\
\text { field }\end{array}$ & & $\begin{array}{c}\text { Distance } \\
(\mathrm{kpc})\end{array}$ & \\
\hline (1) & $\begin{array}{c}(2) \\
\text { (GCCIV) }\end{array}$ & $\begin{array}{c}(3) \\
\text { PGCC }\end{array}$ & $\begin{array}{c}(4) \\
\text { CO-line data }\end{array}$ \\
\hline G005.93-01.00 & - & - & $3.3_{-1.7}^{+6.6}$ \\
\hline G006.01+36.74 & $0.110 \pm 0.010$ & - & -1.1 \\
\hline $\mathrm{G} 010.21+02.40$ & $0.830 \pm 0.400$ & - & $1.0_{-0.5}^{+1.0}$ \\
\hline G014.14-00.55 & - & - & $2.4_{-1.2}^{+2.4}$ \\
\hline G014.15-00.55 & - & - & $2.4_{-12}^{+2.2}$ \\
\hline G016.37-00.61 & - & - & $2.3_{-12}^{+2.2}$ \\
\hline G016.43-00.62 & - & - & $2.3_{-1.2}^{+1.2}$ \\
\hline $\mathrm{G} 016.97+00.28$ & - & - & $2.4_{-1.2}^{+1.4}$ \\
\hline G017.21-01.46 & - & - & $3.6_{-1 .}^{+3.6}$ \\
\hline G023.68+00.58 & - & - & $0.8_{-0.4}^{+0.8}$ \\
\hline G023.69+00.58 & - & - & $0.8_{-0.4}^{+0.8}$ \\
\hline G024.04+00.27 & - & 4.460 & $0.6_{-0.3}^{+0.6}$ \\
\hline $\mathrm{G} 026.54+00.72$ & - & 4.220 & $0.6_{-0.3}^{+0.6}$ \\
\hline G033.73-00.01 & - & 4.220 & $0.7_{-0.4}^{+0.7}$ \\
\hline G034.74-01.39 & - & 4.220 & $0.9_{-0.9}^{+0.9}$ \\
\hline G035.38-01.77 & - & 4.220 & $2.8_{-14}^{+2.8}$ \\
\hline G035.50-00.31 & - & 4.220 & -1.4 \\
\hline G037.93+02.18 & $1.060 \pm 0.790$ & 1.060 & - \\
\hline G039.74+01.98 & $0.990 \pm 0.480$ & - & $2.1_{-1.1}^{+2.1}$ \\
\hline G069.81-01.67 & $1.780 \pm 0.810$ & - & -1.1 \\
\hline G070.40-01.40 & $2.090 \pm 0.830$ & - & - \\
\hline G082.40-01.84 & $1.000_{-0.600}^{+1.000}$ & - & $3.5_{-18}^{+3.5}$ \\
\hline G082.42-01.84 & $1.000_{-0.600}^{+1.000}$ & - & $3.5_{-1.8}^{+3.8}$ \\
\hline G087.07-04.20 & $0.700 \pm 0.100$ & - & -1.8 \\
\hline G089.66-06.62 & $1.210 \pm 1.210$ & - & - \\
\hline G092.04+03.92 & $0.800 \pm 0.100$ & - & - \\
\hline G092.28+03.79 & $0.800 \pm 0.100$ & - & - \\
\hline G105.44+09.88 & - & - & $1.7 \pm 0.3$ \\
\hline G107.18+05.44 & $0.800 \pm 0.100$ & 0.900 & $1.5_{-0.8}^{+1.5}$ \\
\hline G107.26+05.71 & $0.800 \pm 0.100$ & 0.900 & -0 \\
\hline G109.70+02.52 & $0.800 \pm 0.100$ & - & - \\
\hline G109.81+02.72 & $0.800 \pm 0.100$ & 0.700 & $1.4_{-0 .}^{+1.4}$ \\
\hline G139.14-03.23 & $2.500 \pm 0.500$ & - & -0.7 \\
\hline G139.19-3.29 & - & - & 3.2 \\
\hline G159.23-20.09 & - & - & $0.8_{-0.4}^{+0.8}$ \\
\hline G162.46-08.69 & $0.450 \pm 0.023$ & - & - \\
\hline G173.15+02.40 & $2.000 \pm 0.400$ & - & $18.1_{-9.1}^{+18.1}$ \\
\hline G195.73-02.28 & $1.000 \pm 0.500$ & - & $0.9_{-0.9}^{+0.1}$ \\
\hline $\mathrm{G} 202.31+02.53$ & $0.760 \pm 0.100$ & - & $1.0_{-0.5}^{+1.0}$ \\
\hline G215.87-17.50 & $0.425 \pm 0.100$ & - & $1.1_{-0.6}^{+0.1}$ \\
\hline
\end{tabular}

Notes. Distances estimated in Montillaud et al. (2015) (column 2), from the PGCC catalog (Planck Collaboration XXVIII 2016) (column 3), and estimated from $\mathrm{CO}$ line data in this paper (column 4). In case of discrepancy between estimates from the literature and kinematic distances, the former is adopted owing to lower uncertainty. 


\section{Appendix E: FellWalker input values}

The input values used for the FellWalker algorithm are listed in Table E.1. See Sect. 3.3 for details of the RMS values.

Table E.1. Input values used for FellWalker algorithm.

\begin{tabular}{lc}
\hline \hline Variable & Value \\
\hline RMS & Varies \\
MinHeight & $3 \times$ RMS \\
AllowEdge & 0 \\
MinPix & 7 \\
CleanIter & 5 \\
Noise & $1 \times$ RMS \\
FWHMBeam & 1 \\
MaxJump & 3 \\
deconv & True \\
MinDip & $1.5 \times$ RMS \\
\hline
\end{tabular}

Notes. See Section 3.3 for details on RMS values. FlatSlope, VeloRes, and MaxBad values were left to their default values.

\section{Appendix F: Centers and reference regions of SCUBA-2 fields}

Table F.1 lists the central coordinates of each SCUBA-2 field and the coordinates of the reference regions used for background subtraction. Sources without Herschel data (or with insufficient data) do not need background subtraction.

Table F.1. Centers of the SCUBA-2 fields and reference regions.

\begin{tabular}{lcccc}
\hline $\begin{array}{l}\text { SCUBA-2 } \\
\text { field }\end{array}$ & \multicolumn{2}{l}{$\begin{array}{l}\text { Center of } \\
\text { SCUBA-2 field }\end{array}$} & \multicolumn{3}{l}{$\begin{array}{l}\text { Center of } \\
\text { reference region }\end{array}$} \\
\hline & $\begin{array}{c}\text { RA } \\
\left({ }^{\circ}\right)\end{array}$ & $\begin{array}{c}\text { DEC } \\
\left({ }^{\circ}\right)\end{array}$ & $\begin{array}{c}\text { RA } \\
\left({ }^{\circ}\right)\end{array}$ & $\begin{array}{c}\text { DEC } \\
\left({ }^{\circ}\right)\end{array}$ \\
\hline G001.36+20.96 & 248.625 & -15.787 & 248.630 & -15.814 \\
G005.93-01.00 & 270.732 & -24.33 & 270.707 & -24.277 \\
G006.01+36.74 & 238.534 & -2.877 & 238.575 & -2.894 \\
G010.21+02.40 & 269.831 & -18.926 & 269.858 & -18.99 \\
G014.14-00.55 & 274.55 & -16.921 & 274.477 & -16.907 \\
G014.15-00.55 & 274.552 & -16.911 & 274.467 & -16.913 \\
G014.22-00.19 & 274.254 & -16.675 & 274.307 & -16.712 \\
G014.72-00.19 & 274.499 & -16.236 & 274.464 & -16.180 \\
G014.73-00.19 & 274.503 & -16.231 & 274.458 & -16.189 \\
G016.28-00.45 & 275.502 & -14.99 & 275.586 & -15.073 \\
\hline
\end{tabular}

Table F.1. continued.

\begin{tabular}{lcccc}
\hline \hline SCUBA-2 & Center of & \multicolumn{3}{c}{ Center of } \\
field & SCUBA-2 field & reference region \\
\hline G016.37-00.61 & 275.694 & -14.986 & 275.675 & -15.036 \\
G016.43-00.62 & 275.735 & -14.937 & 275.814 & -15.015 \\
G016.97+00.28 & 275.178 & -14.034 & 275.120 & -14.016 \\
G017.23-01.47 & 276.89 & -14.625 & 276.923 & -14.661 \\
G017.38+02.26 & 273.58 & -12.731 & & \\
G023.35-00.26 & 278.69 & -8.639 & 278.625 & -8.601 \\
G023.68+00.58 & 278.094 & -7.958 & 278.041 & -7.955 \\
G023.69+00.58 & 278.101 & -7.947 & 278.046 & -7.944 \\
G024.04+00.27 & 278.541 & -7.782 & & \\
G026.54+00.72 & 279.292 & -5.356 & 279.245 & -5.341 \\
G033.73-00.01 & 283.236 & 0.706 & 283.280 & 0.719 \\
G034.74-01.39 & 284.922 & 0.976 & 284.867 & 0.991 \\
G035.38-01.77 & 285.547 & 1.372 & 285.519 & 1.418 \\
G035.50-00.31 & 284.311 & 2.146 & 284.320 & 2.187 \\
G037.93+02.18 & 283.195 & 5.438 & 283.188 & 5.438 \\
G039.74+01.98 & 284.2 & 6.962 & 284.214 & 6.9292 \\
G057.12+03.63 & 290.992 & 23.131 & & \\
G069.81-01.67 & 303.393 & 31.365 & 303.413 & 31.337 \\
G070.40-01.40 & 303.513 & 32.015 & 303.492 & 32.067 \\
G074.13+00.11 & 304.504 & 35.955 & 304.472 & 36.008 \\
G082.40-01.84 & 312.858 & 41.406 & 312.902 & 41.379 \\
G082.42-01.84 & 312.874 & 41.425 & 312.884 & 41.394 \\
G087.07-04.20 & 319.447 & 43.31 & 319.444 & 43.2741 \\
G089.66-06.62 & 324.313 & 43.358 & 324.352 & 43.350 \\
G091.88+04.17 & 315.126 & 52.522 & 315.200 & 52.512 \\
G092.04+03.92 & 315.616 & 52.475 & 315.669 & 52.472 \\
G092.28+03.79 & 316.033 & 52.569 & 315.951 & 52.573 \\
G093.51-04.31 & 326.236 & 47.624 & 326.131 & 47.631 \\
G105.44+09.88 & 325.816 & 66.131 & 325.935 & 66.152 \\
G107.18+05.44 & 335.376 & 63.626 & 335.266 & 63.605 \\
G107.26+05.71 & 335.206 & 63.903 & 335.116 & 63.884 \\
G109.70+02.52 & 343.374 & 62.326 & 343.240 & 62.329 \\
G109.81+02.72 & 343.391 & 62.546 & 343.267 & 62.570 \\
G139.14-03.23 & 42.617 & 55.849 & 42.487 & 55.859 \\
G159.23-20.09 & 53.363 & 31.127 & 53.404 & 31.134 \\
G162.46-08.69 & 65.388 & 37.567 & 65.434 & 37.620 \\
G171.53-14.91 & 67.179 & 26.832 & 67.221 & 26.843 \\
G172.89+02.28 & 84.235 & 36.177 & & \\
G173.15+02.40 & 84.533 & 36.019 & & \\
G178.28-00.60 & 84.784 & 30.081 & 84.720 & 30.108 \\
G195.02.28 & 92.751 & 14.171 & 92.761 & 14.198 \\
G0 & 88.416 & -10.401 & & \\
\hline
\end{tabular}

Notes. (Columns 2-3) Right ascension and declination (J2000) of the center of the SCUBA-2 maps. (Columns 4-5) The right ascension and declination (J2000) for the centers of empty reference regions used for background subtraction. The background subtraction uses a radius of $0.02^{\circ}$. 


\section{Appendix G: Comparison of clump-finding algorithms}

Table G.1. Input values used for ClumpFind and GaussClumps algorithms.

\begin{tabular}{lccc}
\hline \hline $\begin{array}{l}\text { ClumpFind } \\
\text { Variable }\end{array}$ & Value & $\begin{array}{c}\text { GaussClumps } \\
\text { Variable }\end{array}$ & Value \\
\hline RMS & Varies & RMS & Varies \\
Allowedge & 0 & Extracols & 0 \\
Minpix & 16 & Minpix & 3 \\
Maxbad & 0.05 & Maxbad & 0.05 \\
FWHMbeam & 2 & FWHMbeam & 2 \\
Velores & 2 & Velores & 2 \\
IDLalg & 1 & Wmin & 0.05 \\
DeltaT & 2×RMS & Npeak & 9 \\
Naxis & 3 & Maxskip & 10 \\
Noise & $2 \times$ RMS & Nsigma & 3 \\
Tlow & $2.5 \times$ RMS & Thresh & 5 \\
& & Width & 2 \\
\hline
\end{tabular}

Notes. See section 3.3 for details on RMS values, which are the same as used for FellWalker analysis. The values used are the same as those used in (Li et al. 2020).

There are many methods for finding dense clumps, for example, ClumpFind (Williams et al. 1994), GaussClumps (Stutzki \& Guesten 1990; Stutzki 2014), and AstroDendro ${ }^{7}$ (Robitaille et al. 2019). Compared to the others, AstroDendro is more recent but has been used in several studies in the past year alone (i.e. Kong 2019; Hall \& Battersby 2019). ClumpFind is an older, more established algorithm that has been used in many of the GBS papers (Tosaki et al. 2017; Figueira et al. 2019).

We examined the importance of the clump-finding algorithm used by comparing ClumpFind and GaussClumps with FellWalker. The parameters used in the ClumpFind and GaussClumps algorithms are listed in Table G.1. Figure G.1 shows cumulative histograms of the number of clumps per field found using all three algorithms. The results for GaussClumps and ClumpFind are very similar. FellWalker found 529 clumps, but ClumpFind found 779 and GaussClumps 785. Likewise, with ClumpFind each field has between 1 and 54 clumps, and both ClumpFind and GaussClumps have a mean of just under 15 clumps per field, compared to a mean of 10 clumps per field with FellWalker. This is also visible in Fig. G.2, which shows the 11 FellWalker, 14 ClumpFind, and 16 GaussClumps clumps found in the field G202.31+02.53. Compared to FellWalker, the other two algorithms divided the central structure into smaller pieces but detected fewer clumps in other parts of the map.

Cheng et al. (2018) compared the results of AstroDendro with those of ClumpFind and also find a difference in number of clumps; however, this difference is slightly smaller than that which we find for field G202.31+02.53. ClumpFind is also found by the authors to overestimate the number of clumps when

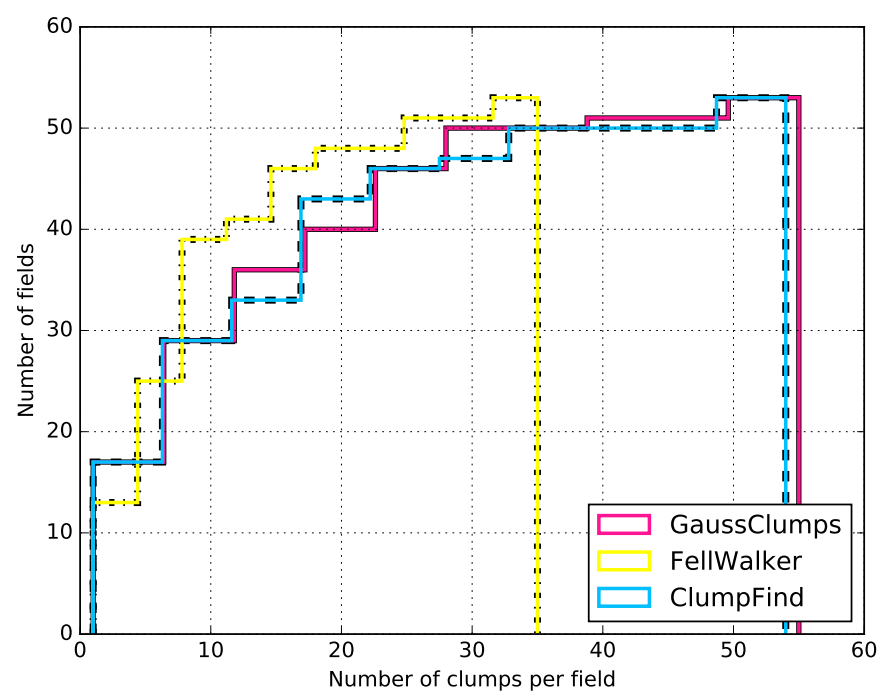

Fig. G.1. Cumulative histograms of number of clumps found in each field using the GaussClumps (pink), FellWalker (yellow), and ClumpFind (blue) algorithms.

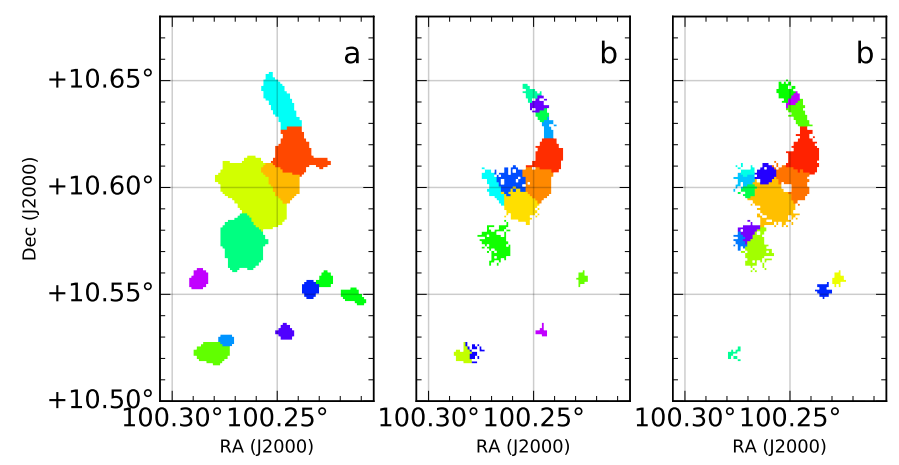

Fig. G.2. Clumps produced by FellWalker (a), ClumpFind (b), and GaussClumps (c) for field G202.31+02.53.

compared with other clump-finding algorithms. Fellwalker, Dendrograms, and GaussClumps have been ranked as the best clump-finding algorithms by Li et al. (2020). These three had better detection completeness on a large sample of simulated clumps. Furthermore the authors found that FellWalker was best at extracting the total flux of the clumps.

The differences in the size and the number of the detected clumps would have a significant consequences for the analysis. The derived masses and radii of ClumpFind- or GaussClumpsderived clumps would be smaller, thus probably also decreasing the number of clumps that would be classified as prestellar. In addition to this, the parameters used and the determination of the RMS value also affects the number and type of clumps detected.

\footnotetext{
7 http://WwW.dendrograms .org/
} 


\section{Appendix H: Extended emission maps}
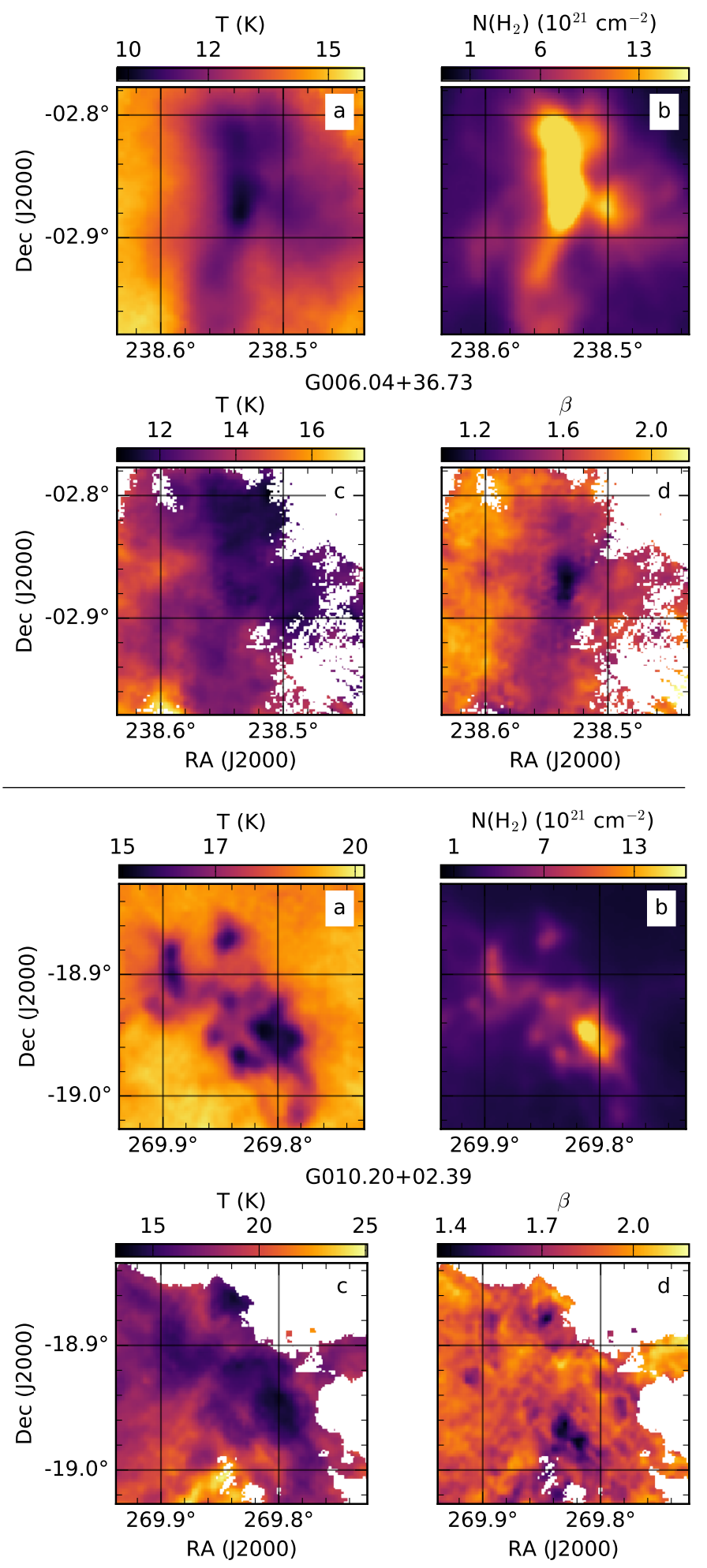

Fig. H.1. Extended emission maps of the full Herschel fields. The name of the field is written below frames a and $\mathrm{b}$. The two fields are divided by the horizontal line. (a) $250-500 \mu \mathrm{m}$ temperature with constant $\beta=1.8$. (b) $250-500 \mu \mathrm{m}$ column density $N\left(\mathrm{H}_{2}\right)$ with constant $\beta=1.8$. (c) 160 $500 \mu \mathrm{m}$ temperature with background subtraction and varied spectral index. (d) $160-500 \mu \mathrm{m}$ spectral index with background subtraction.

We show temperature, column density, and spectral index maps for Herschel fields with SPIRE and PACS $160 \mu \mathrm{m}$ data in Figs. H.1-H.12. The temperatures and column densities of the
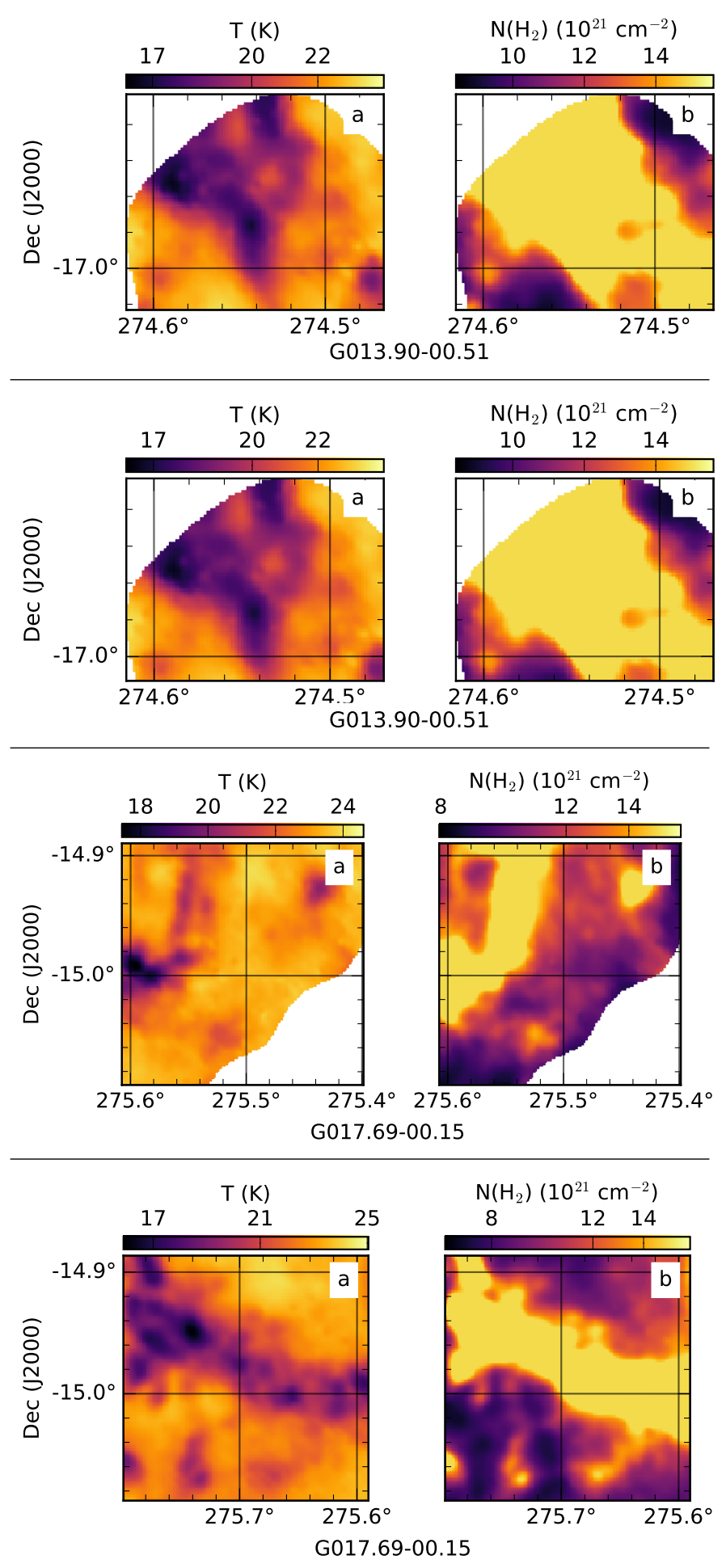

Fig. H.2. Same as Fig. H.1 for fields G014.14-00.55 and G014.1500.55, both located on Herschel field G013.90, and G016.28-00.45 and G016.37-00.61, both located on Herschel field G017.69.

upper frames are calculated using the SPIRE bands and a fixed value of $\beta=1.8$. The lower frames correspond to fits that include the PACS $160 \mu \mathrm{m}$ band and have spectral index as a free parameter. All Herschel fields are cropped to the size of the SCUBA2 field. Fields with insufficient PACS $160 \mu \mathrm{m}$ data only show frames a and b. Pixels with negative PACS $160 \mu \mathrm{m}$ intensities are masked in frames $\mathrm{c}$ and $\mathrm{d}$. 

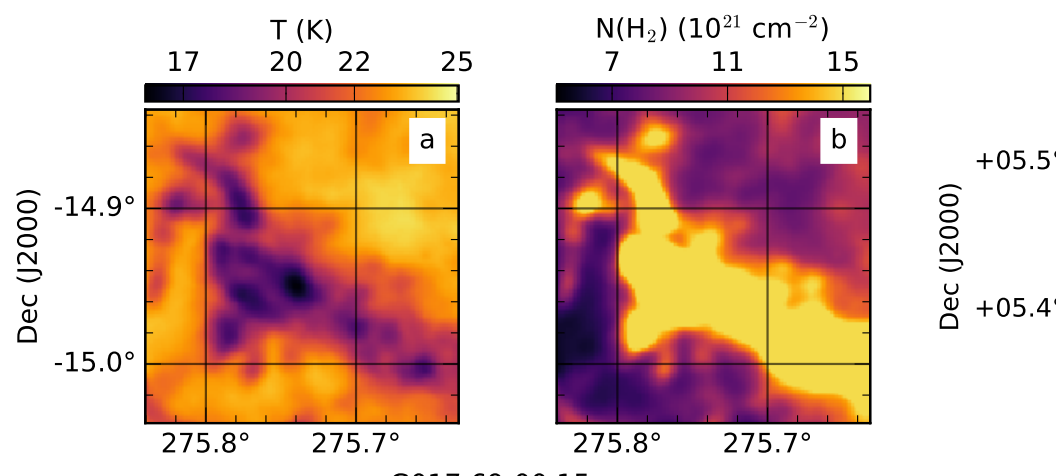

$\mathrm{T}(\mathrm{K})$

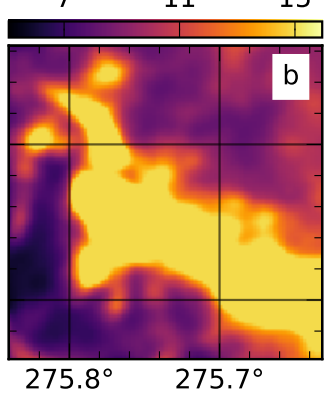

G017.69-00.15

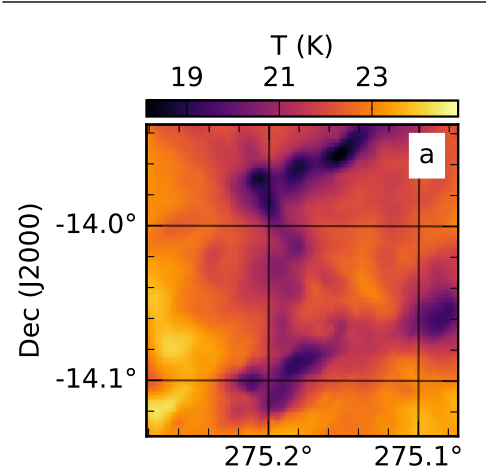

$\mathrm{N}\left(\mathrm{H}_{2}\right)\left(10^{21} \mathrm{~cm}^{-2}\right)$
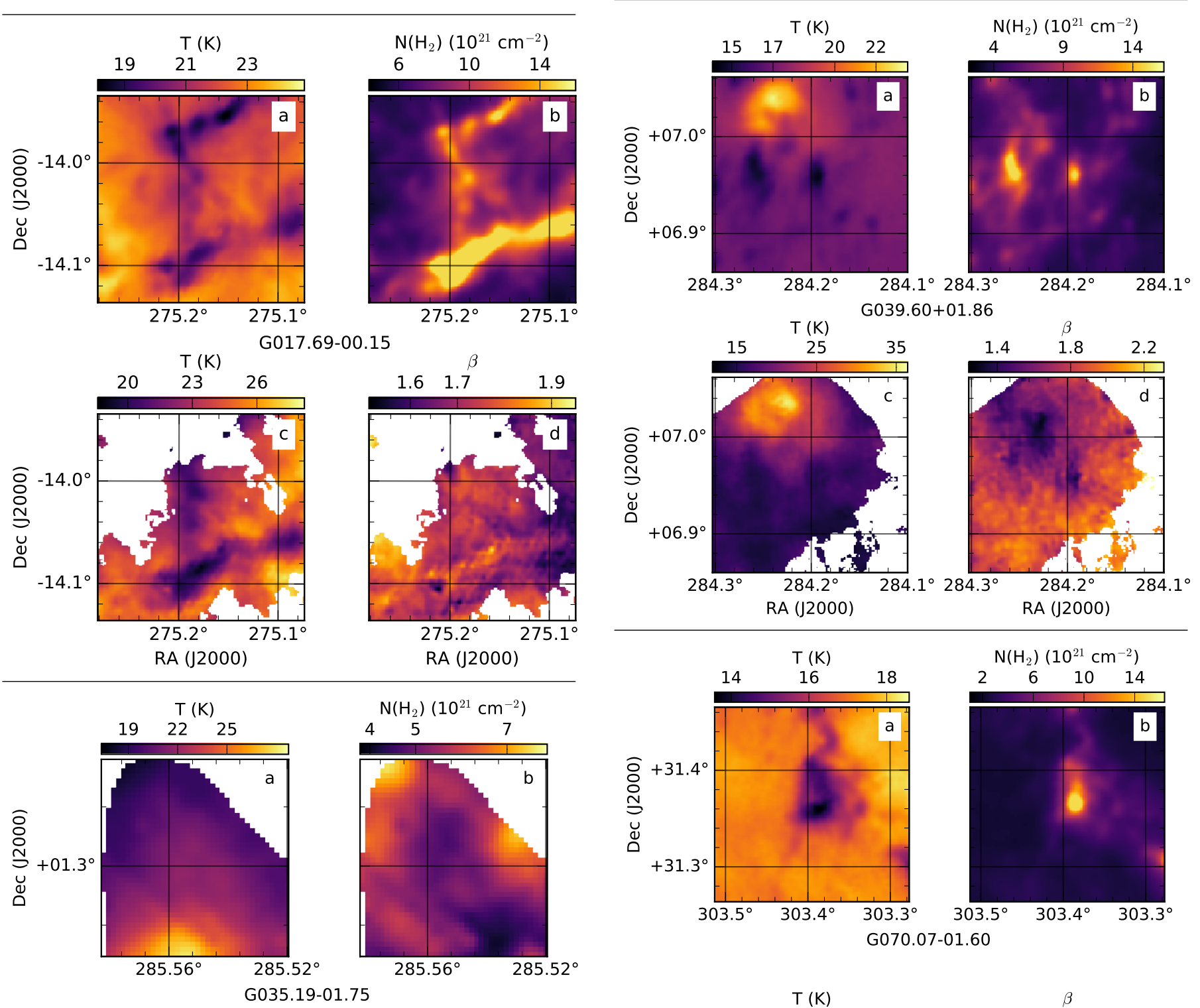

Fig. H.3. Same as Fig. H.1 for fields G016.43-00.62, G016.97+00.28 (both located on Herschel field G017.69), and G035.38-01.77.
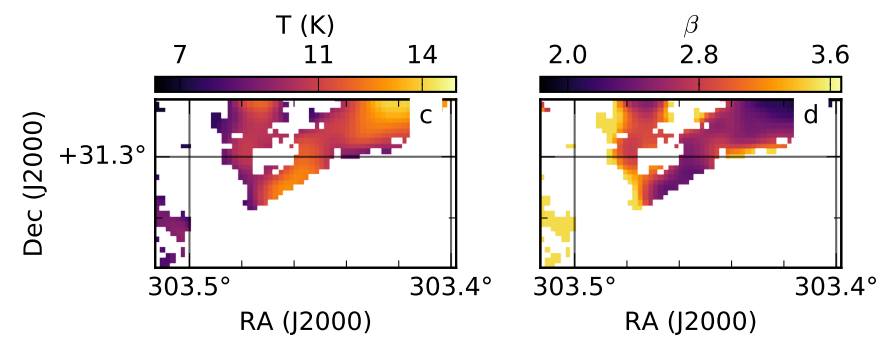

Fig. H.4. Same as Fig. H.1 for fields G037.93+02.18, G039.74+01.98, and G069.81-01.67. 
E. Mannfors et al.: Characterization of dense Planck clumps observed with Herschel and SCUBA-2
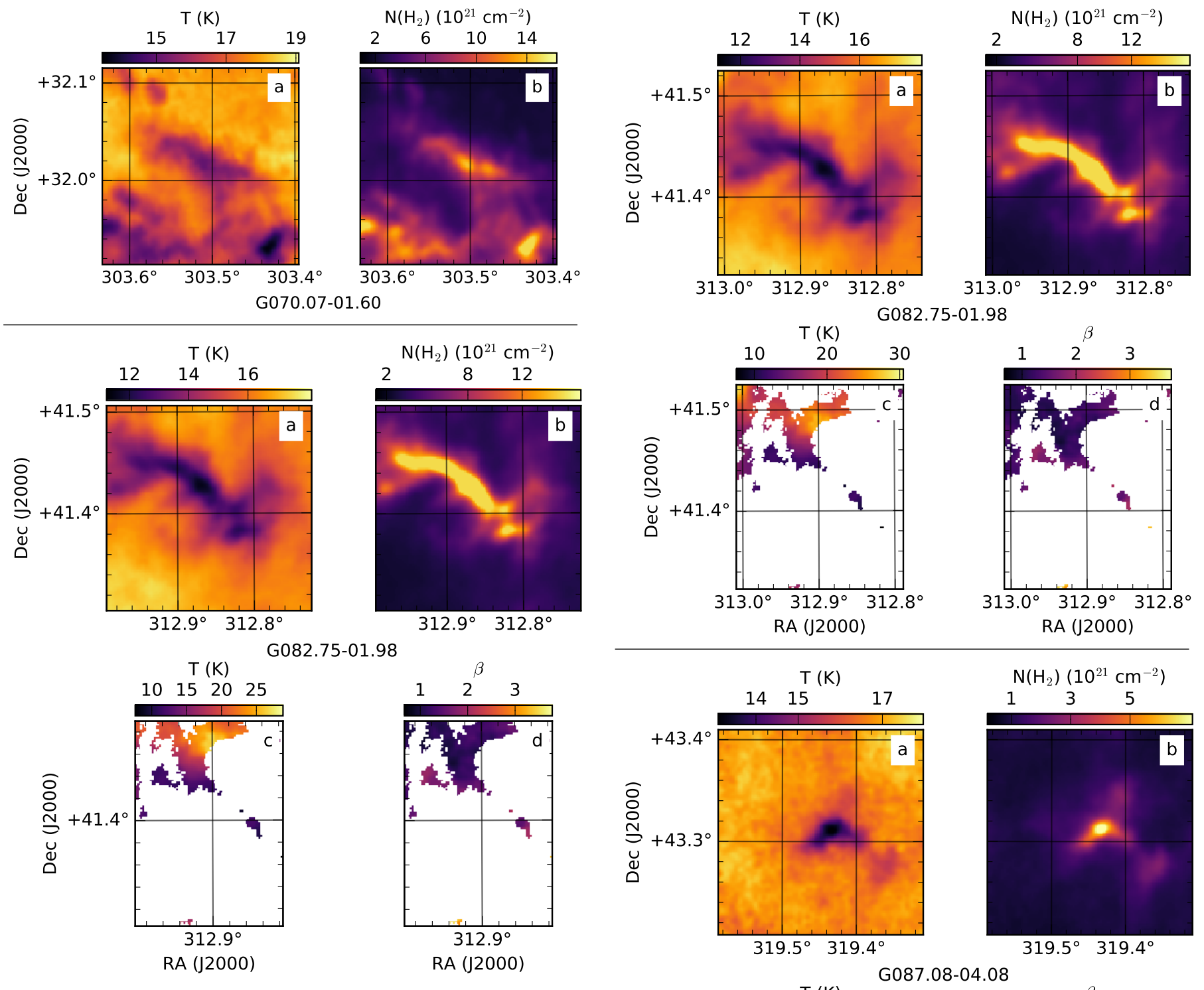

Fig. H.5. Same as Fig. H.1 for fields G070.40-01.40 and G082.40-
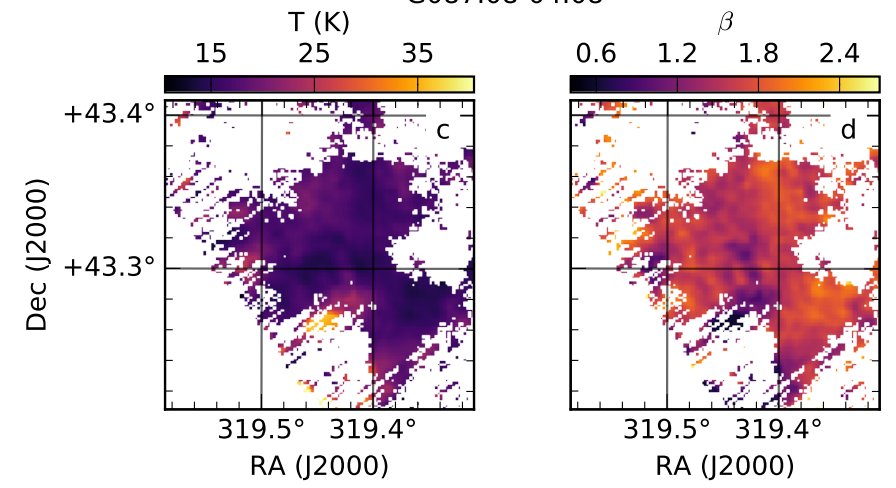

Fig. H.6. Same as Fig. H.1 for fields G082.42-01.84 and G087.0704.20 . 

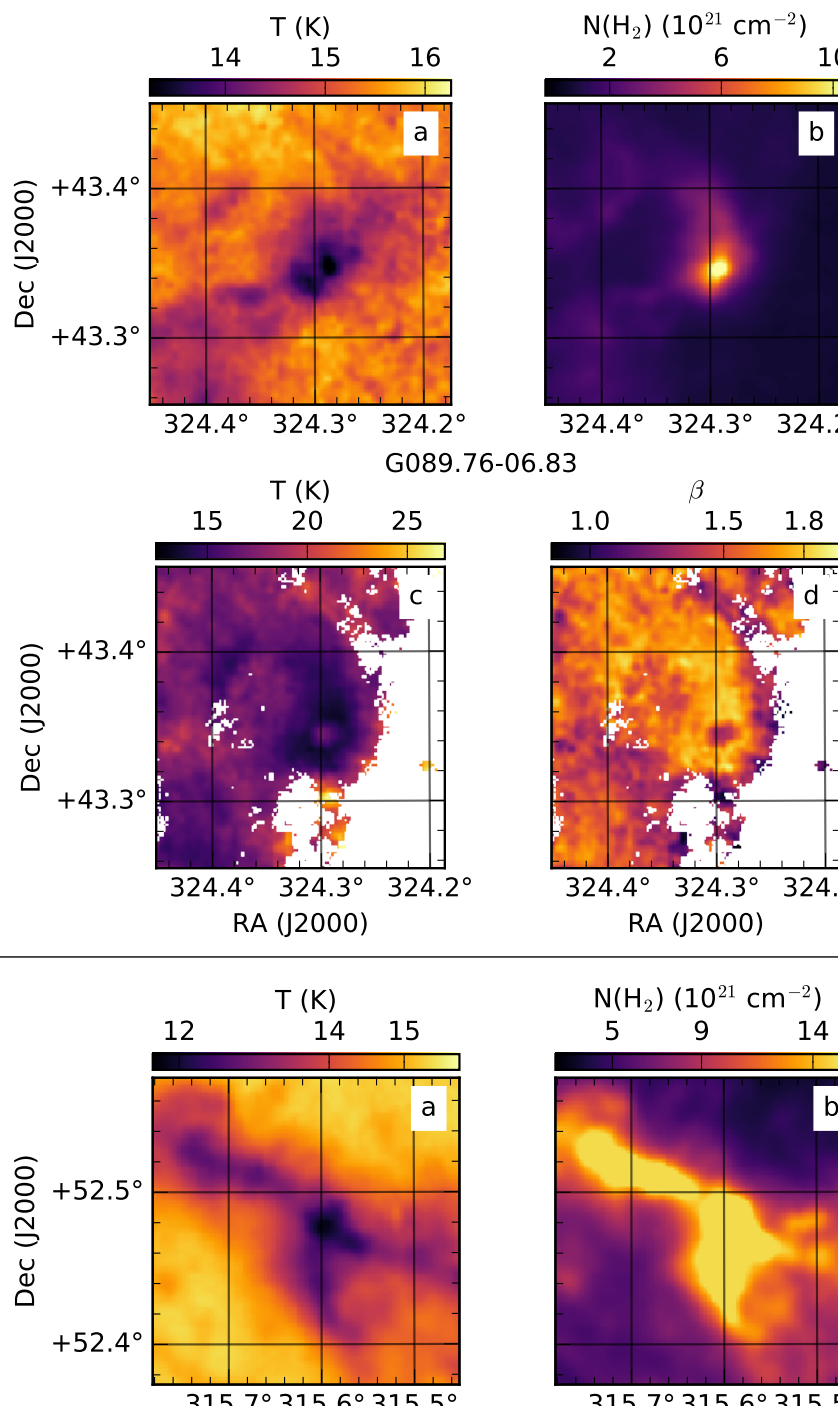

$315.7^{\circ} 315.6^{\circ} 315.5^{\circ}$

$\mathrm{G} 092.12+03.88$
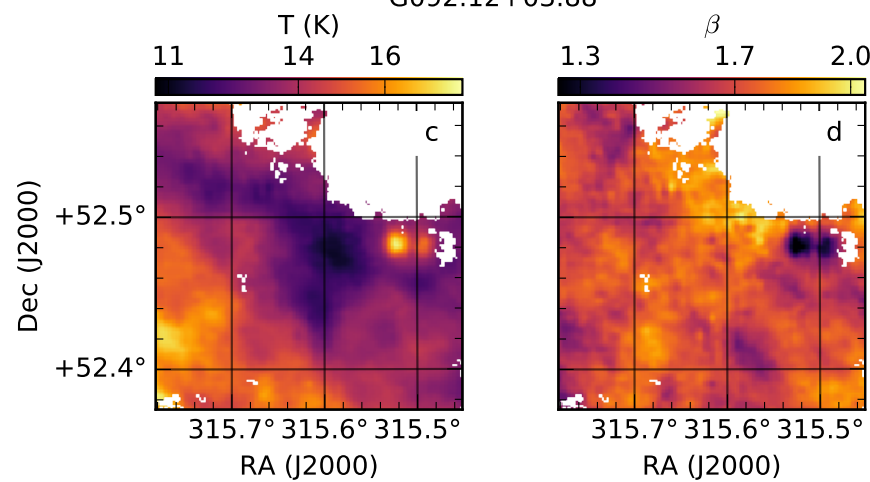

Fig. H.7. Same as Fig. H.1 for fields G089.66-06.62 and G092.04+ 03.92 .
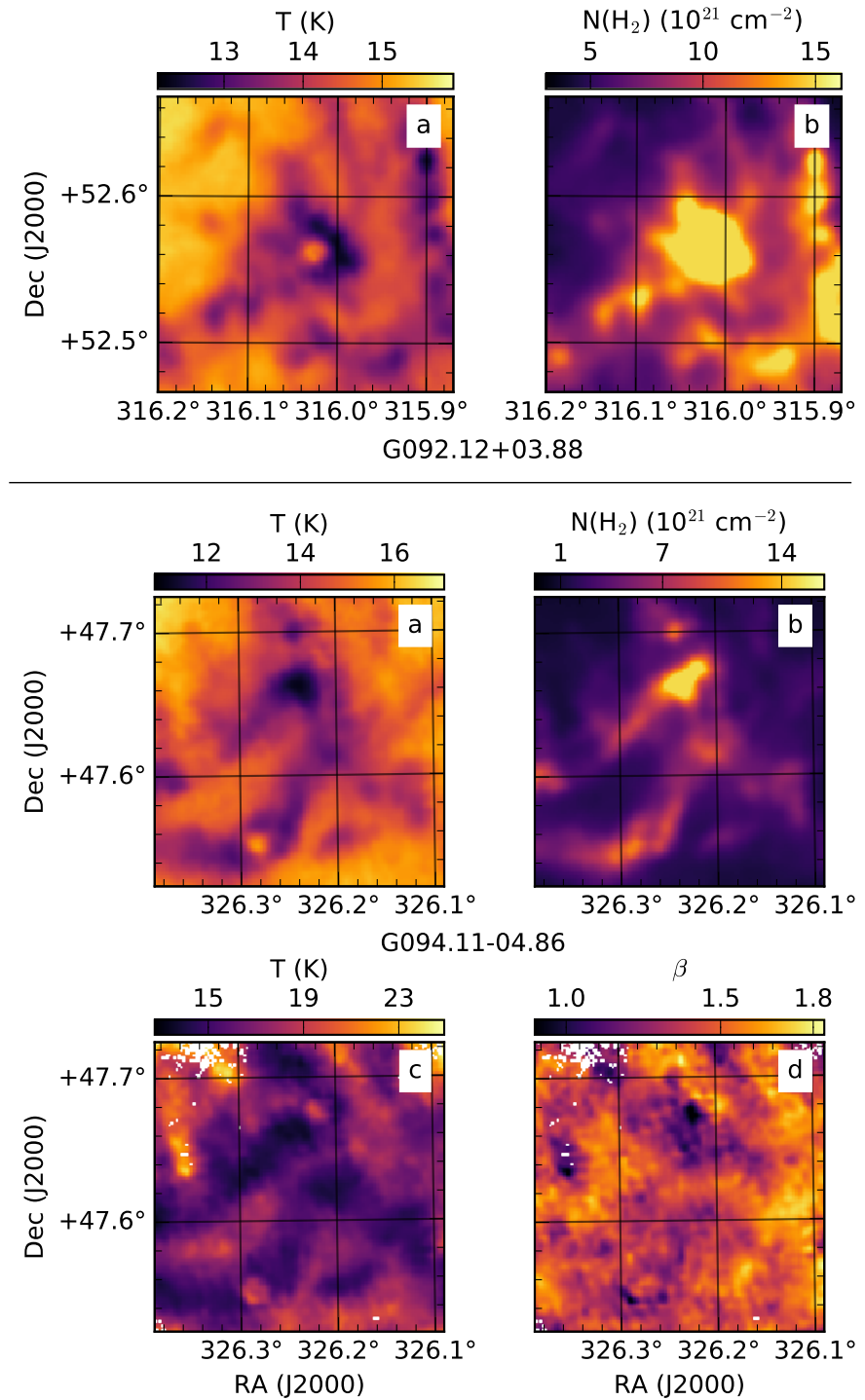

Fig. H.8. Same as Fig. H.1 for fields G092.28+03.79 and G093.5104.31 . 
E. Mannfors et al.: Characterization of dense Planck clumps observed with Herschel and SCUBA-2
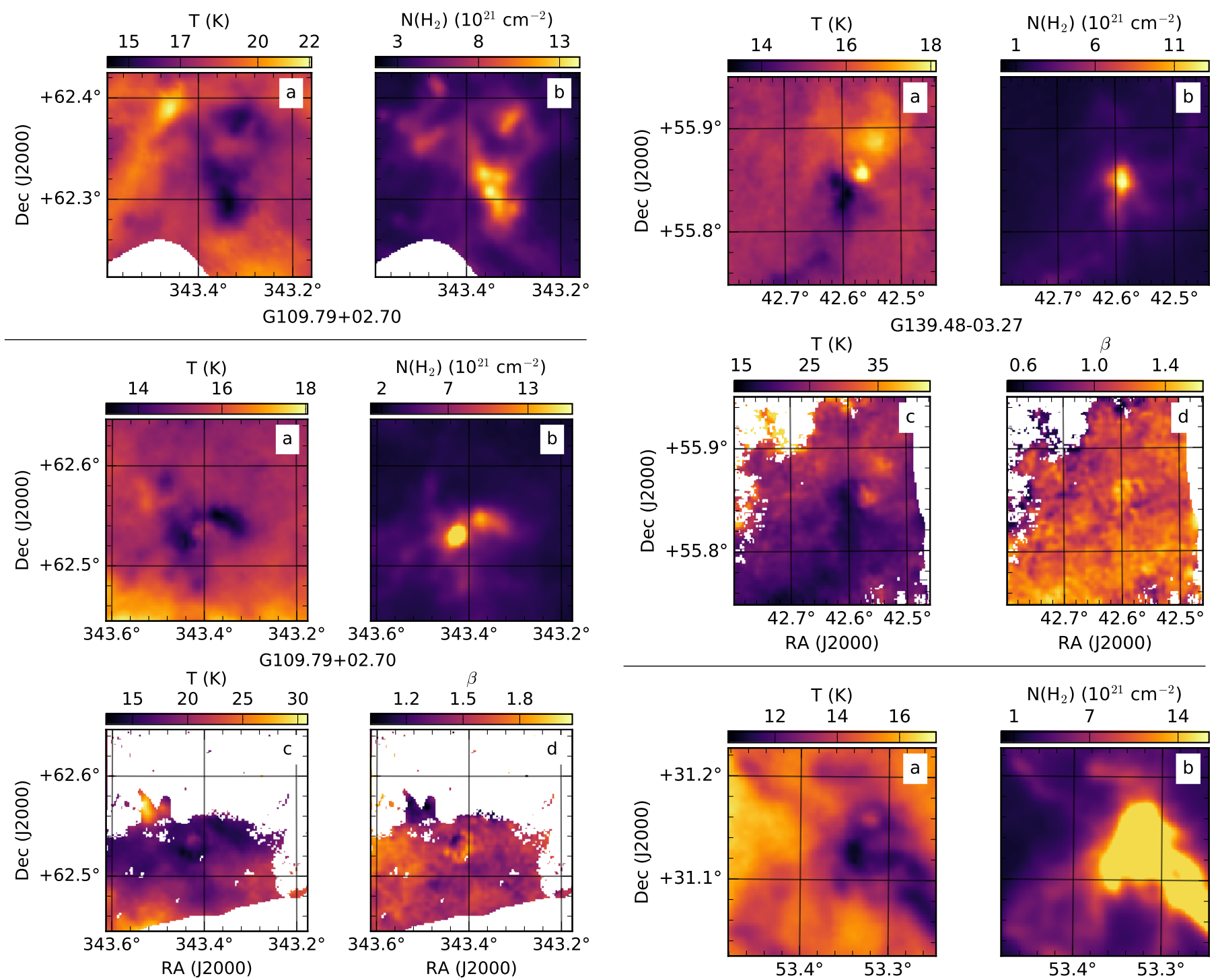

Fig. H.9. Same as Fig. H.1 for fields G109.70+02.52 and G109.81+ 02.72 .

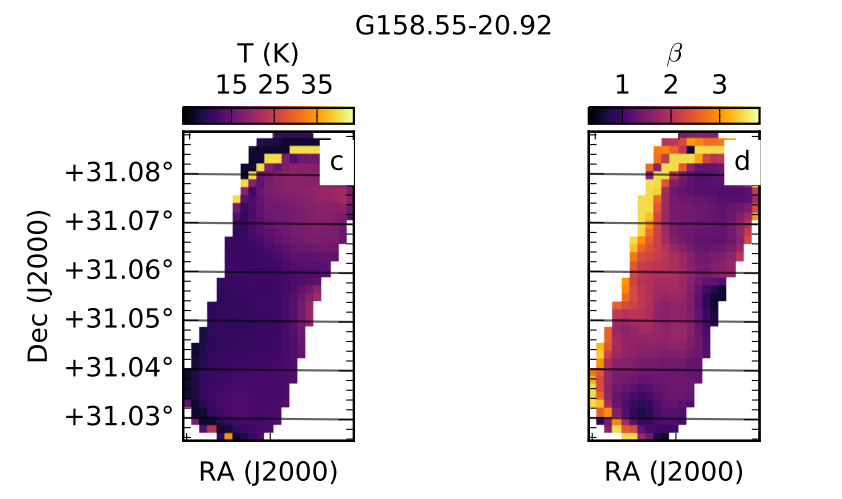

Fig. H.10. Same as Fig. H.1 for fields G139.14-03.23 and G159.2320.09 . 

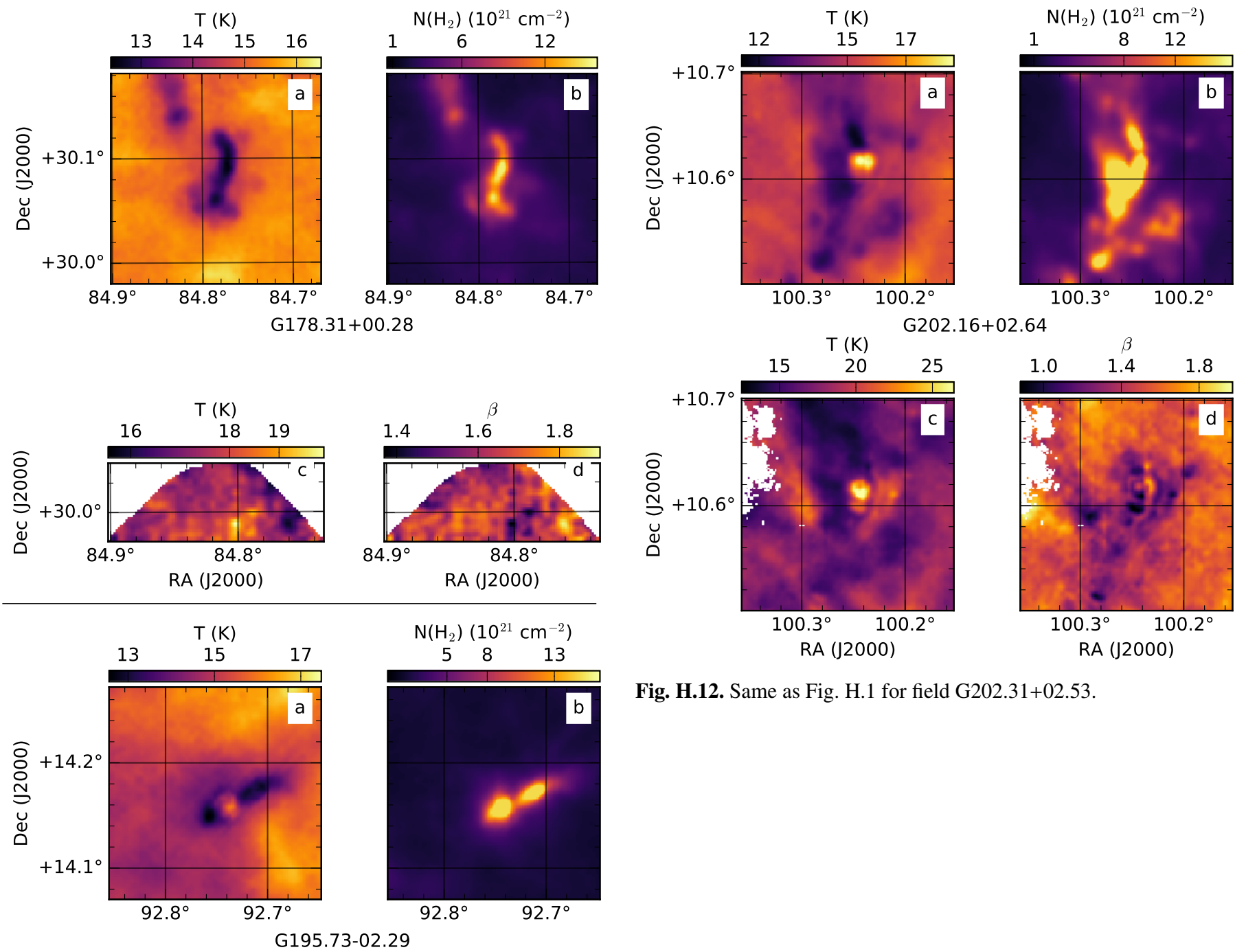

Fig. H.12. Same as Fig. H.1 for field G202.31+02.53.
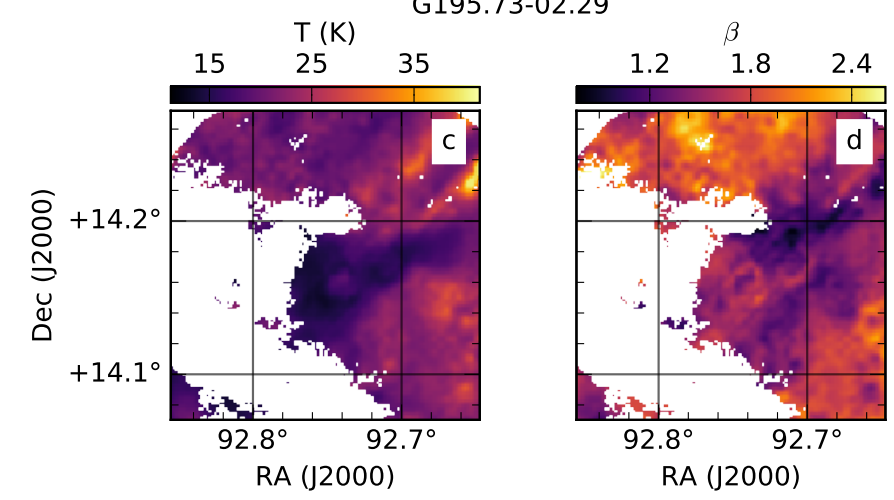

Fig. H.11. Same as Fig. H.1 for fields G178.28-00.60 and G195.7302.28 . 


\section{Appendix I: Herschel spectra}

In Fig. I.1 we show as an example the SEDs of nine randomly selected clumps used for studying $850 \mu \mathrm{m}$ excess. See Sect. 4.4 for further details.
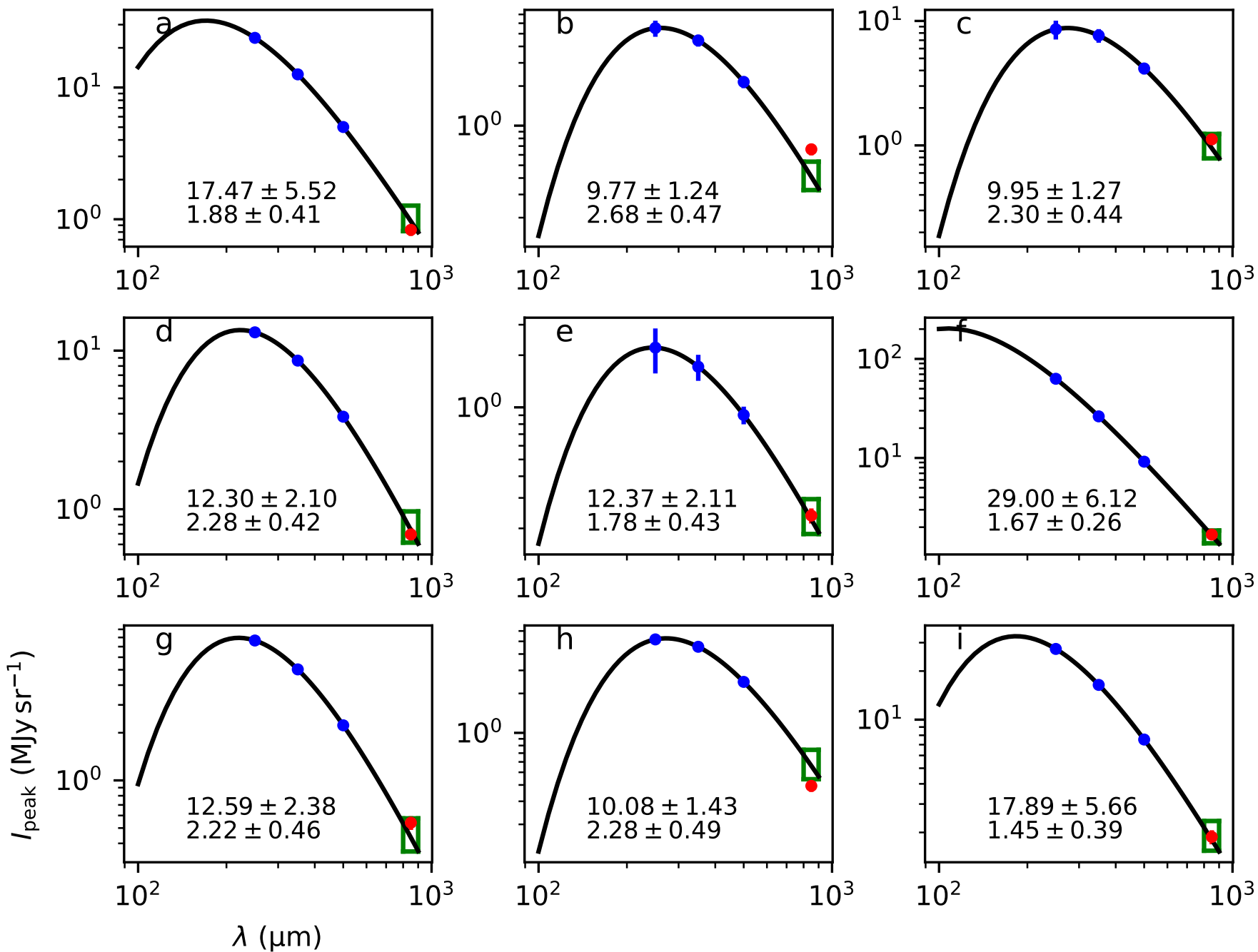

Fig. I.1. SEDs of the peak pixels of nine randomly-selected clumps with $\mathrm{S} / \mathrm{N}>3$ in all bands. The blue dots are at 250,350 , and $500 \mu \mathrm{m}$, respectively. The green box indicates the 5\%-95\% confidence interval of the extrapolated $850 \mu \mathrm{m}$ value, and the red dot the observed value at $850 \mu \mathrm{m}$. 


\section{Appendix J: YSOs and clumps on $850 \mu \mathrm{m}$ intensity maps}

All candidate YSOs associated with the clumps are shown on SCUBA-2 intensity maps in Figs. J.1-J.5. The clumps are plotted with white ellipses.
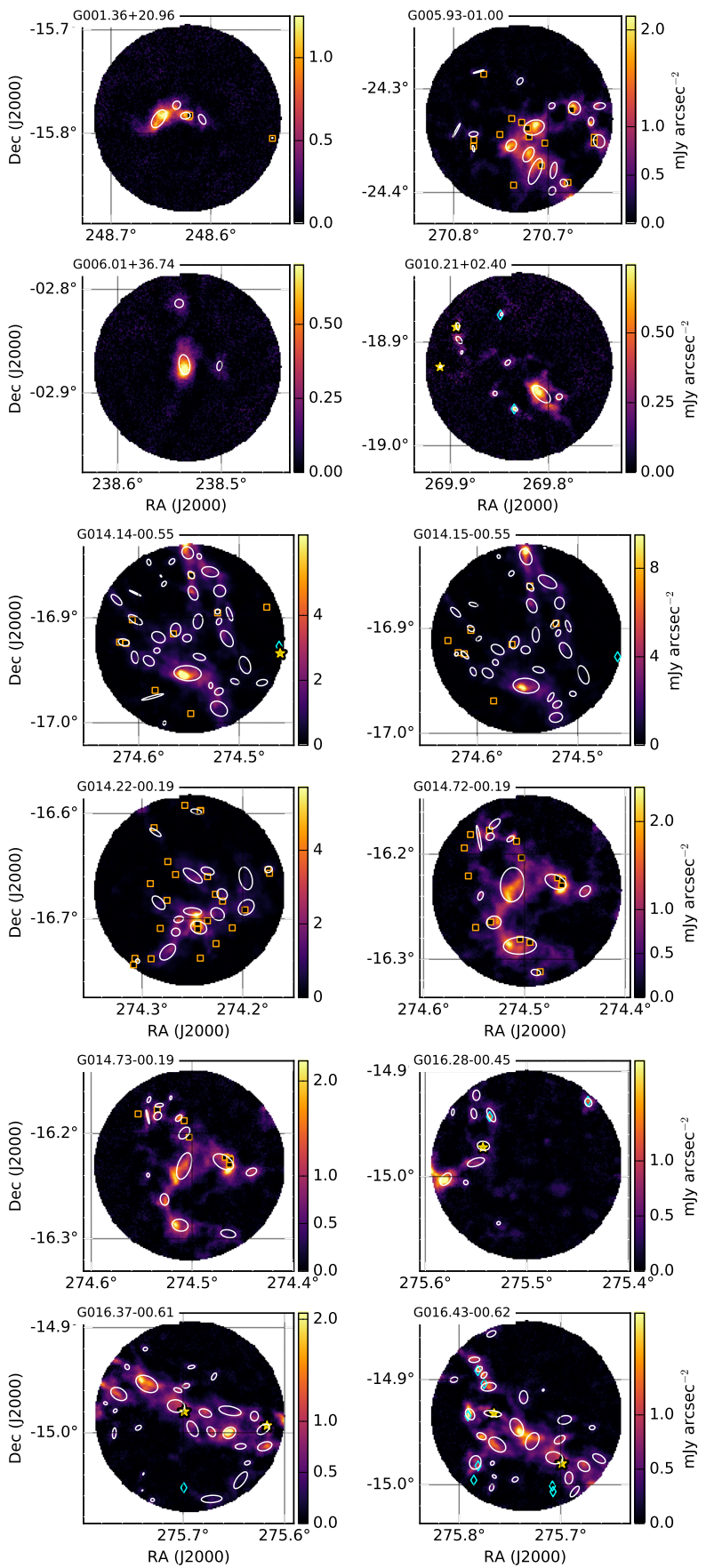

Fig. J.1. Surface brightness maps of the SCUBA-2 $850 \mu \mathrm{m}$ intensity. Clumps found by FellWalker are plotted with white ellipses. The orange squares with black centers show the locations of unconfirmed YSOs for which Herschel intensity ratio was not possible to calculate. The cyan diamonds show those YSOs that do not show significant increase in $\Delta C$, and yellow stars those YSOs that have been confirmed with $\Delta C$. Field YSOs are not plotted and no distinction is made between YSOs from different catalogs. The field names are in the top left.
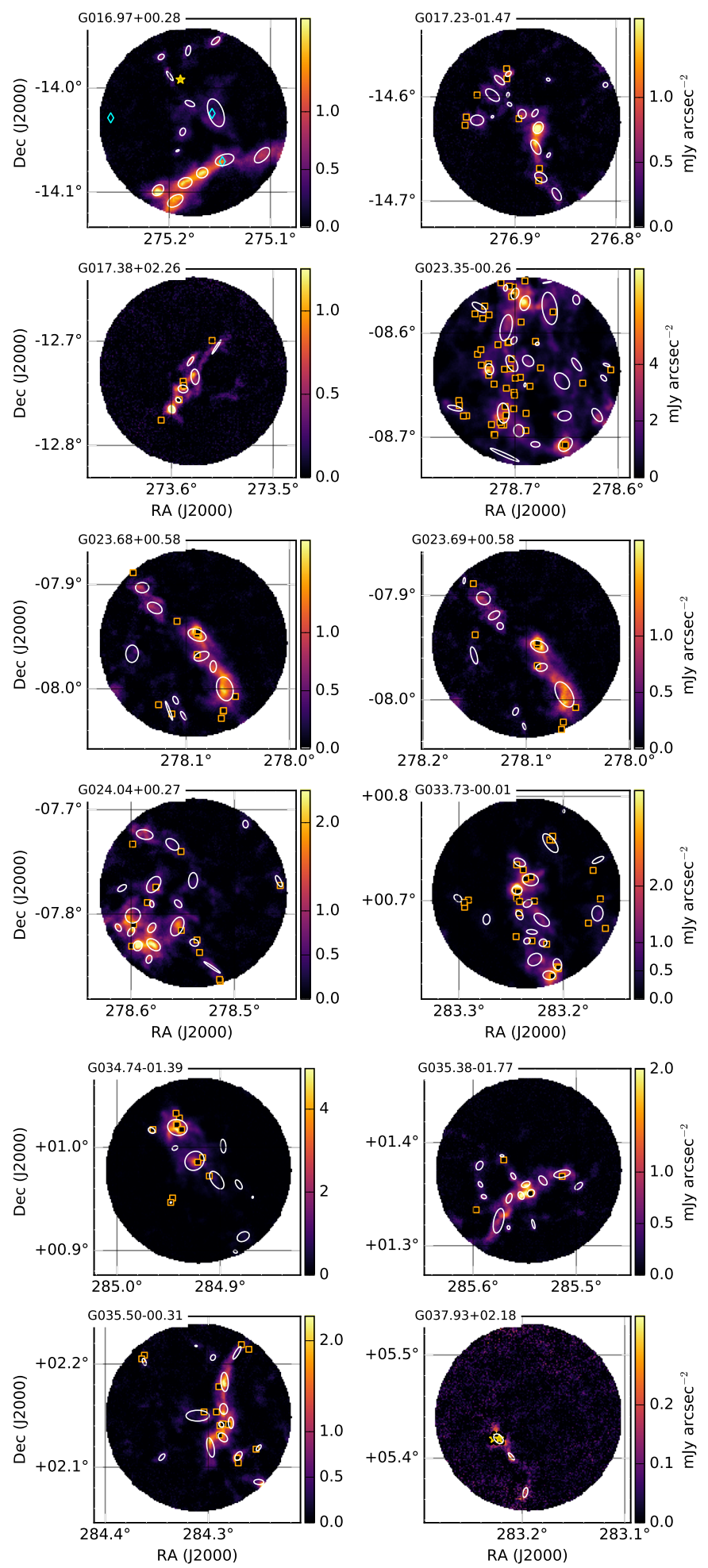

Fig. J.2. Same as Fig. J.1 for fields G016.97+00.28 - G037.93+02.18. 
E. Mannfors et al.: Characterization of dense Planck clumps observed with Herschel and SCUBA-2
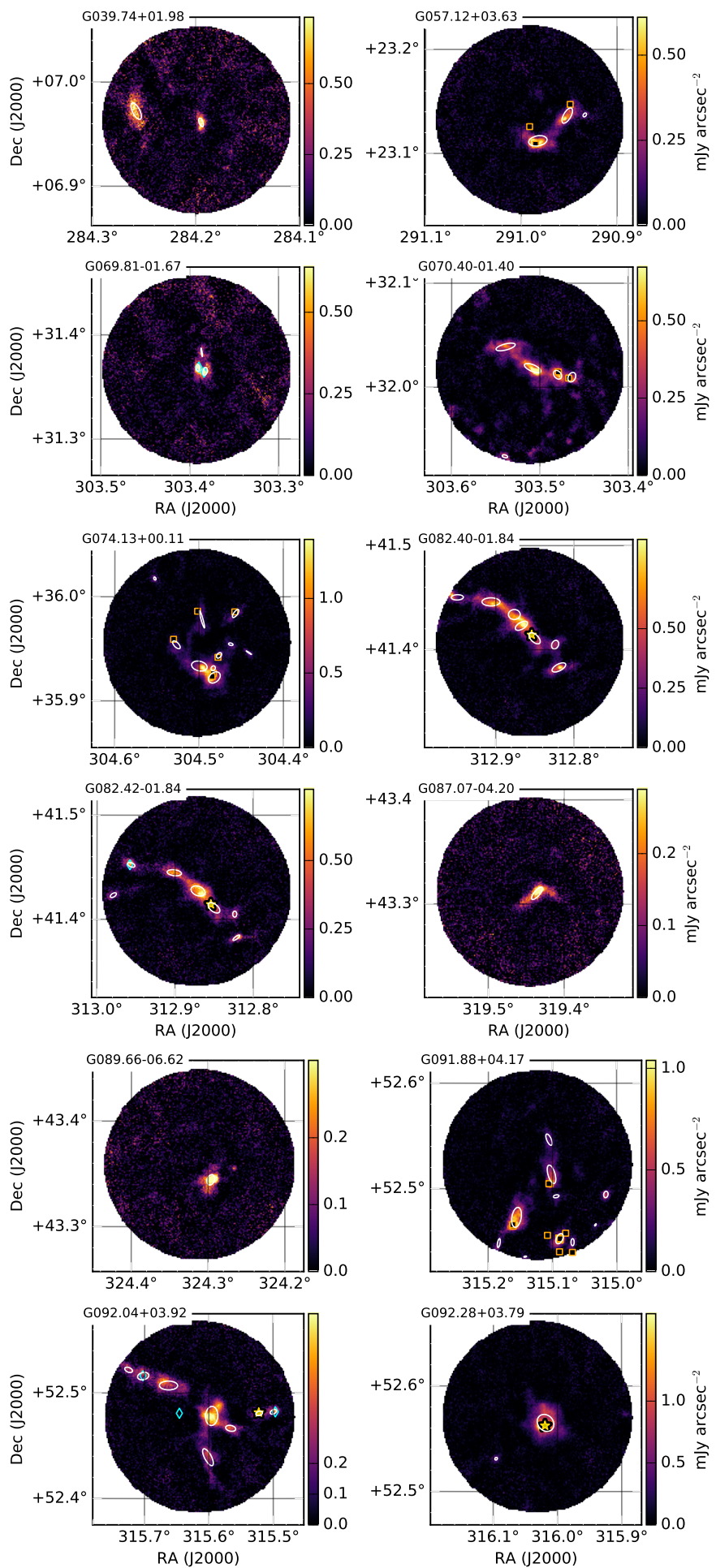

Fig. J.3. Same as Fig. J.1 for fields G039.74+01.98 - G092.28+03.79.
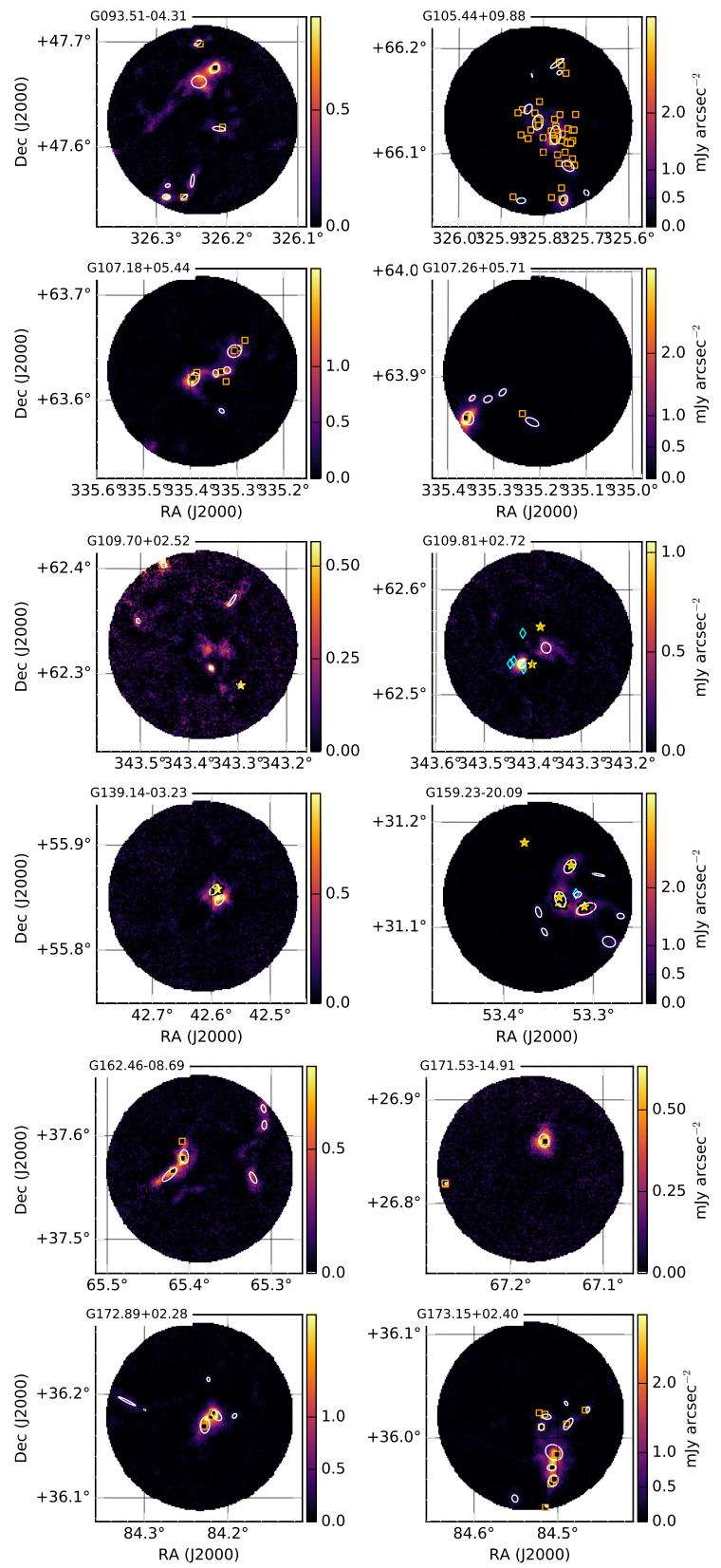

Fig. J.4. Same as Fig. J.1 for fields G093-04.31 - G173.15+02.40.
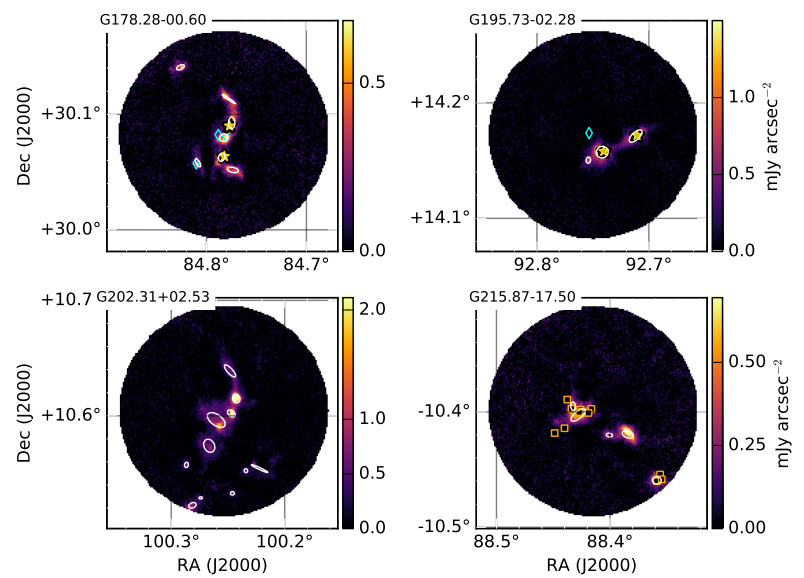

Fig. J.5. Same as Fig. J.1 for fields G178.28-00.60 - G215.87-17.50. 


\section{Appendix K: Catalog of derived clump properties}

We derive up to 14 properties for each clump. The temperature, flux density, $I_{250}, N\left(\mathrm{H}_{2}\right)$, and $N_{\mathrm{YSO}, \Delta \mathrm{C}}$ were not possible to calculate for clumps without Herschel data. The effective radius, mass, and virial parameters were not possible to calculate for clumps without distance estimates. These are denoted with a "-" when a value was not available.

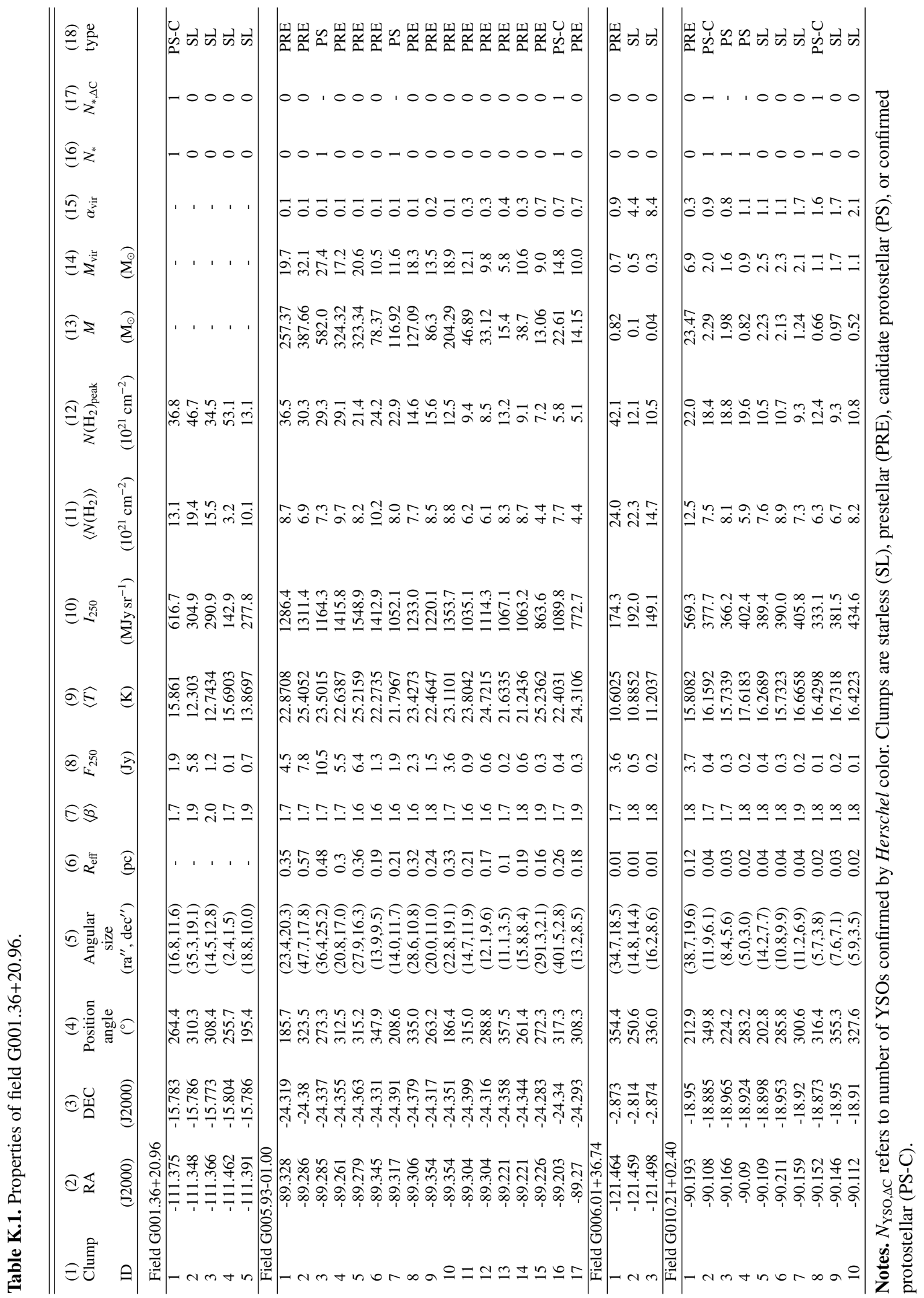


E. Mannfors et al.: Characterization of dense Planck clumps observed with Herschel and SCUBA-2

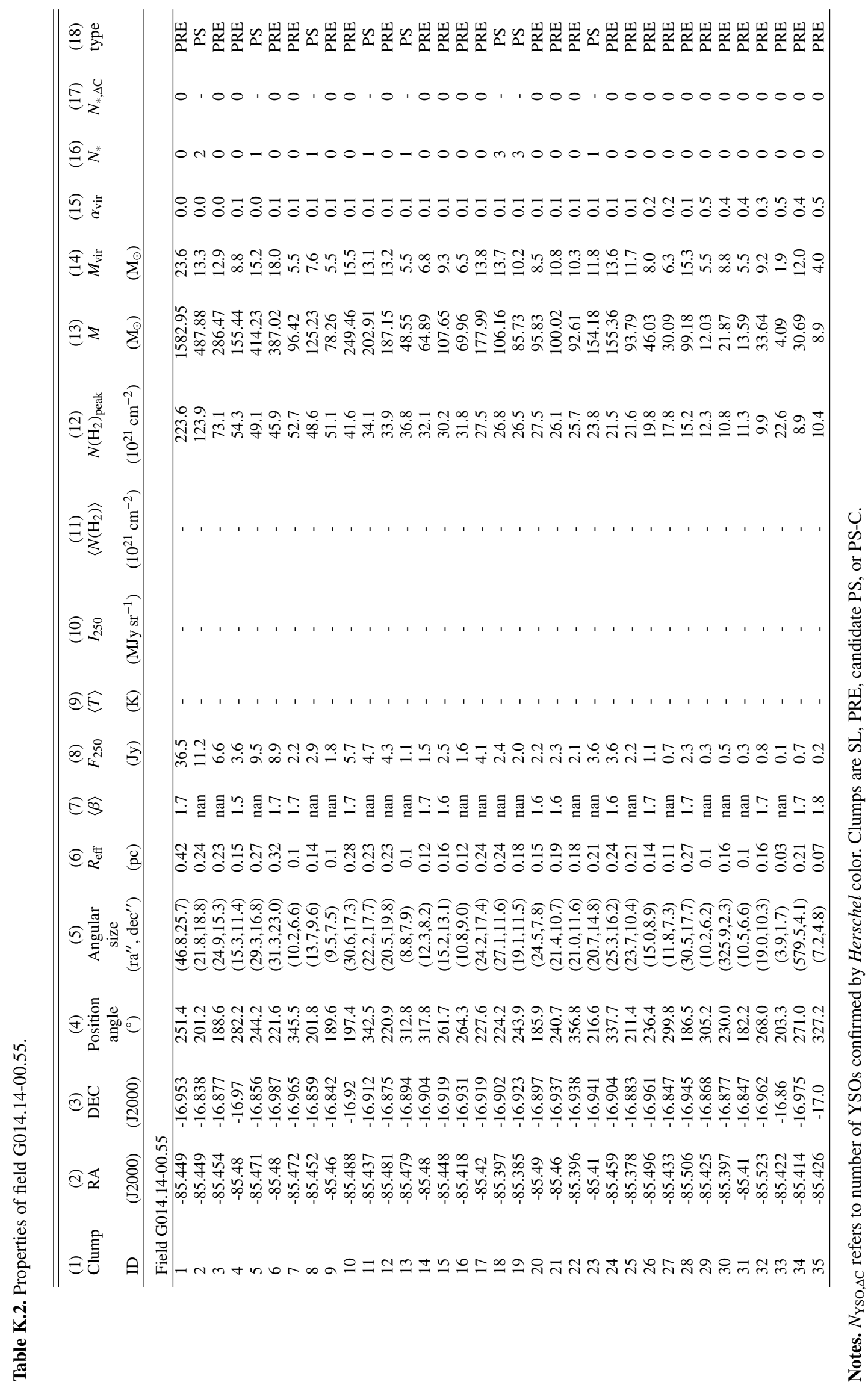




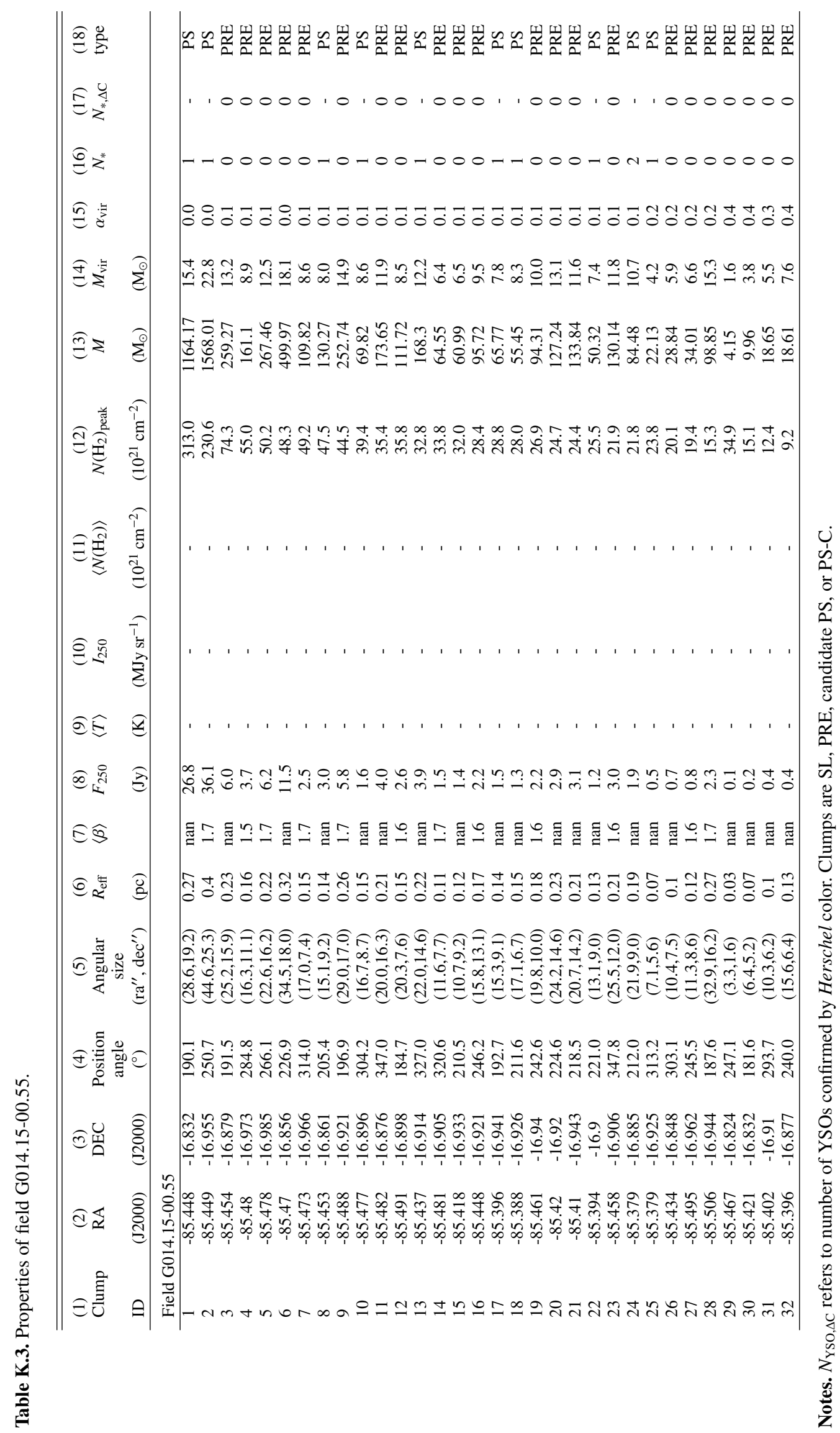


E. Mannfors et al.: Characterization of dense Planck clumps observed with Herschel and SCUBA-2

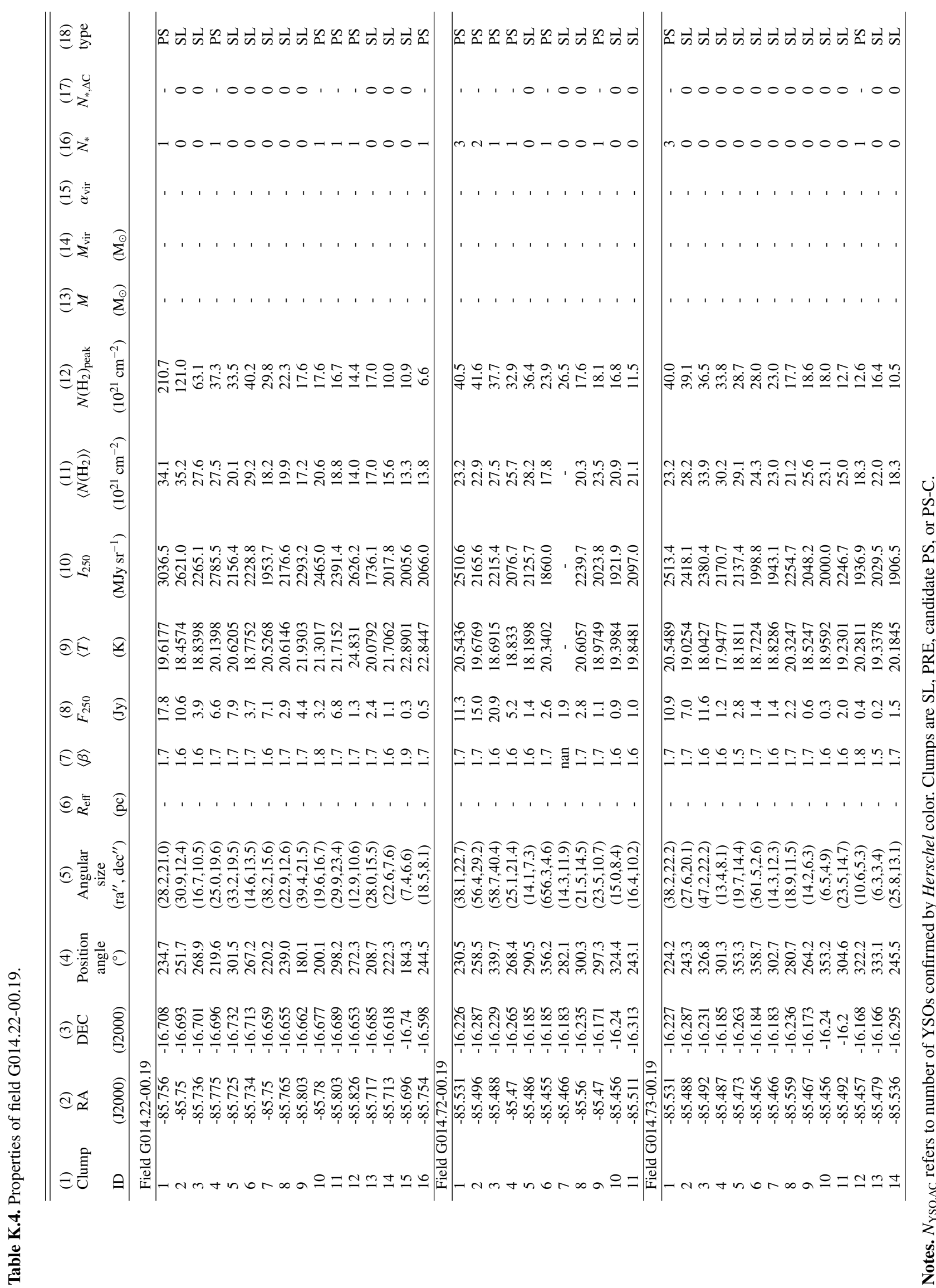


A\&A 654, A123 (2021)

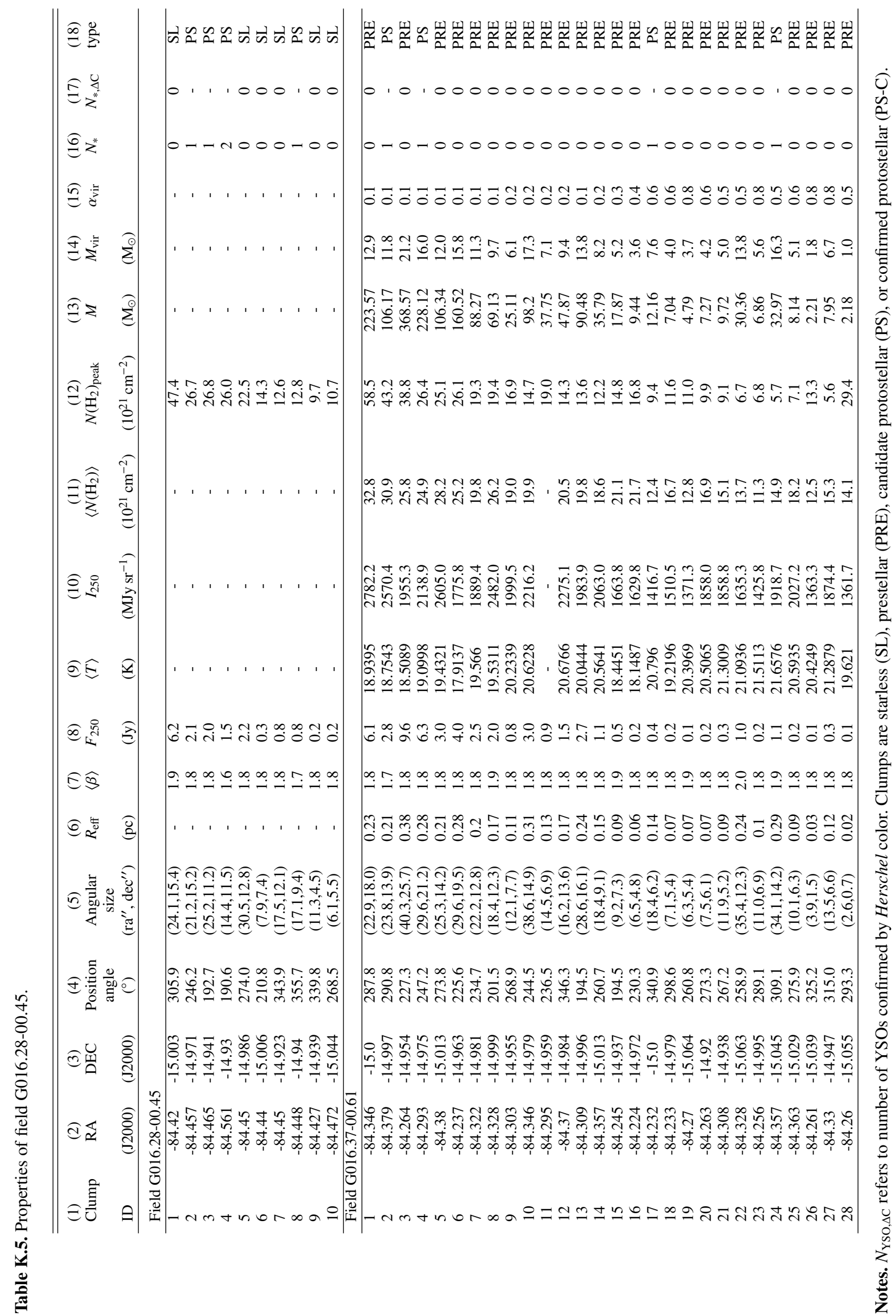


E. Mannfors et al.: Characterization of dense Planck clumps observed with Herschel and SCUBA-2

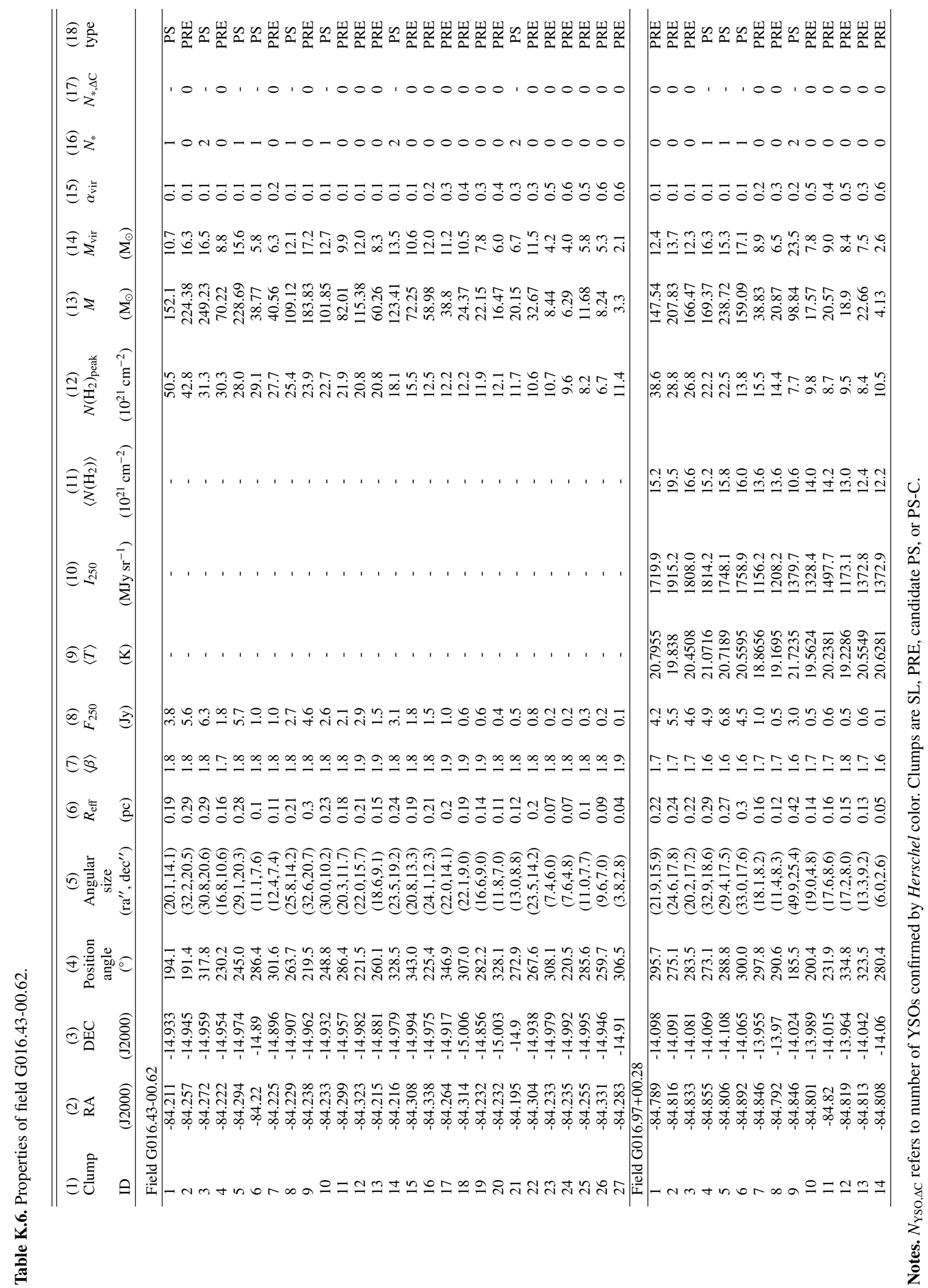




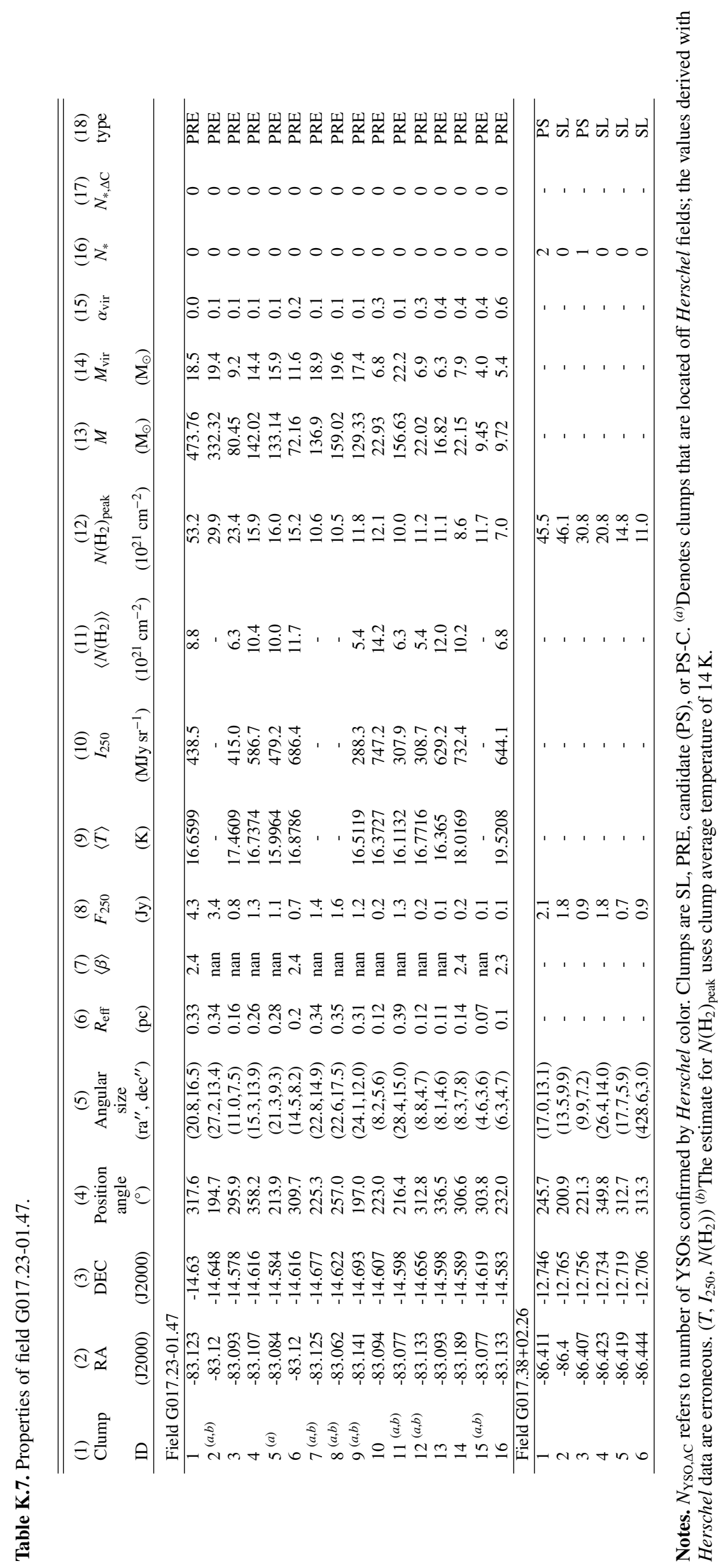


E. Mannfors et al.: Characterization of dense Planck clumps observed with Herschel and SCUBA-2

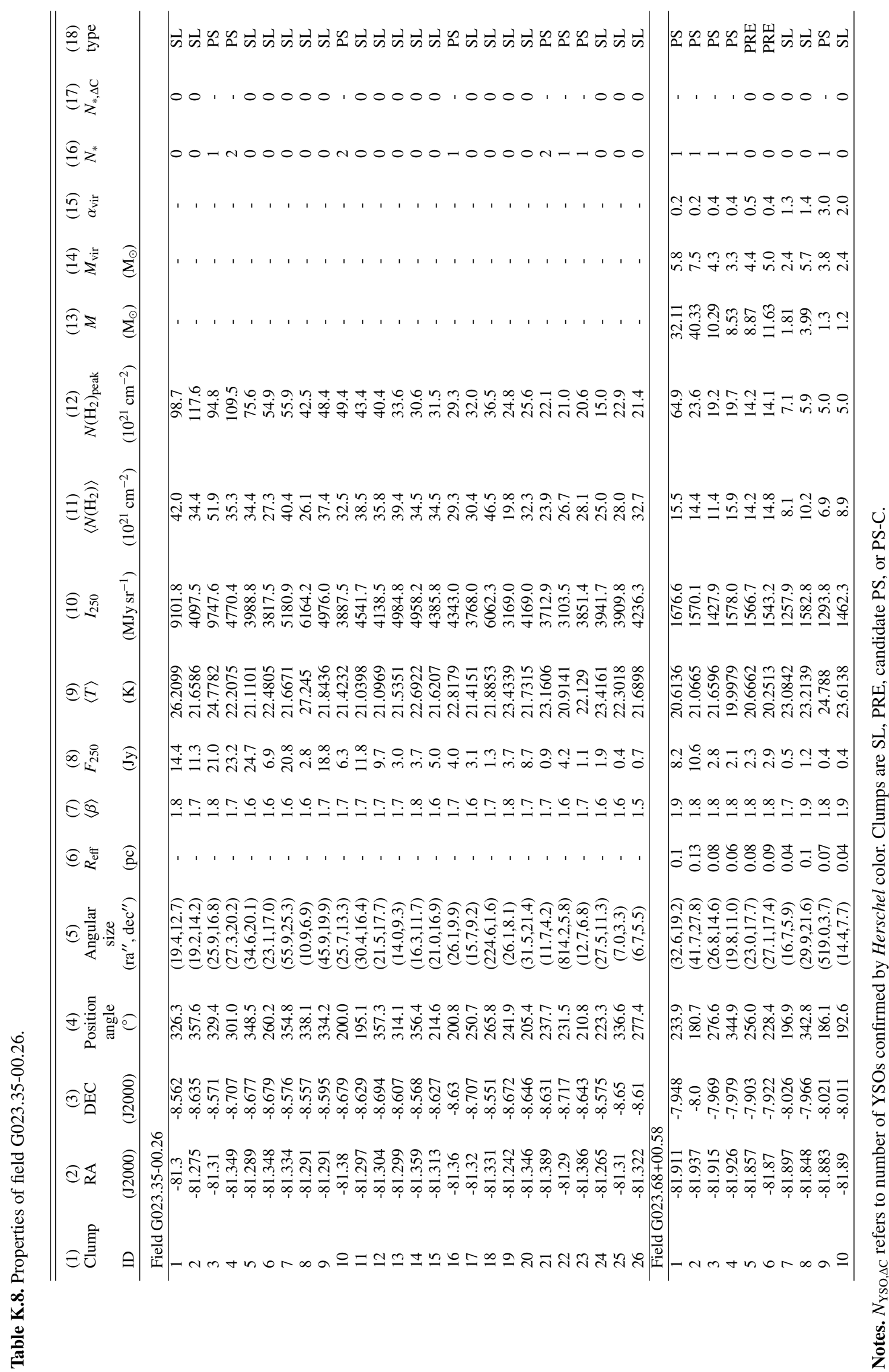




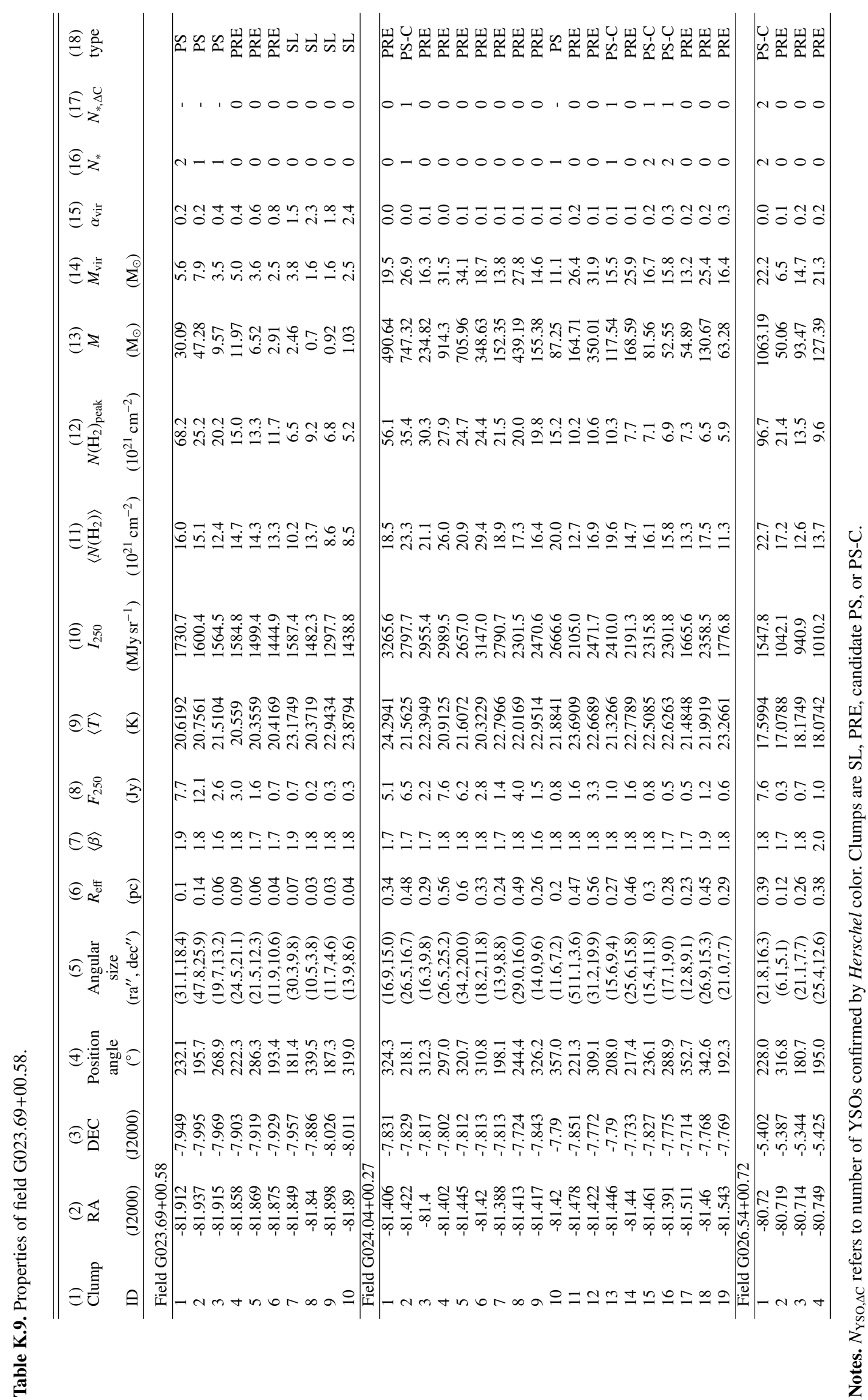


E. Mannfors et al.: Characterization of dense Planck clumps observed with Herschel and SCUBA-2

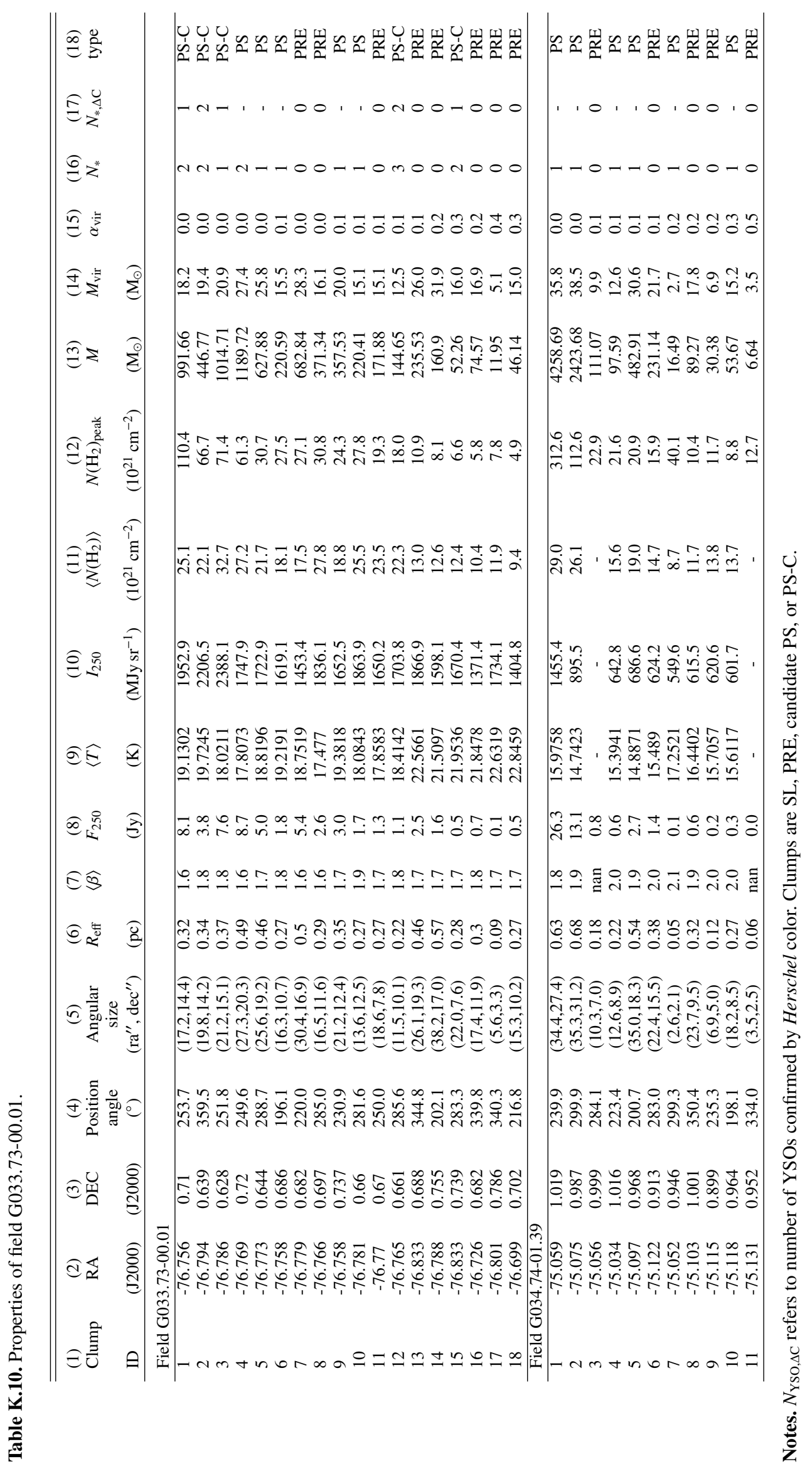




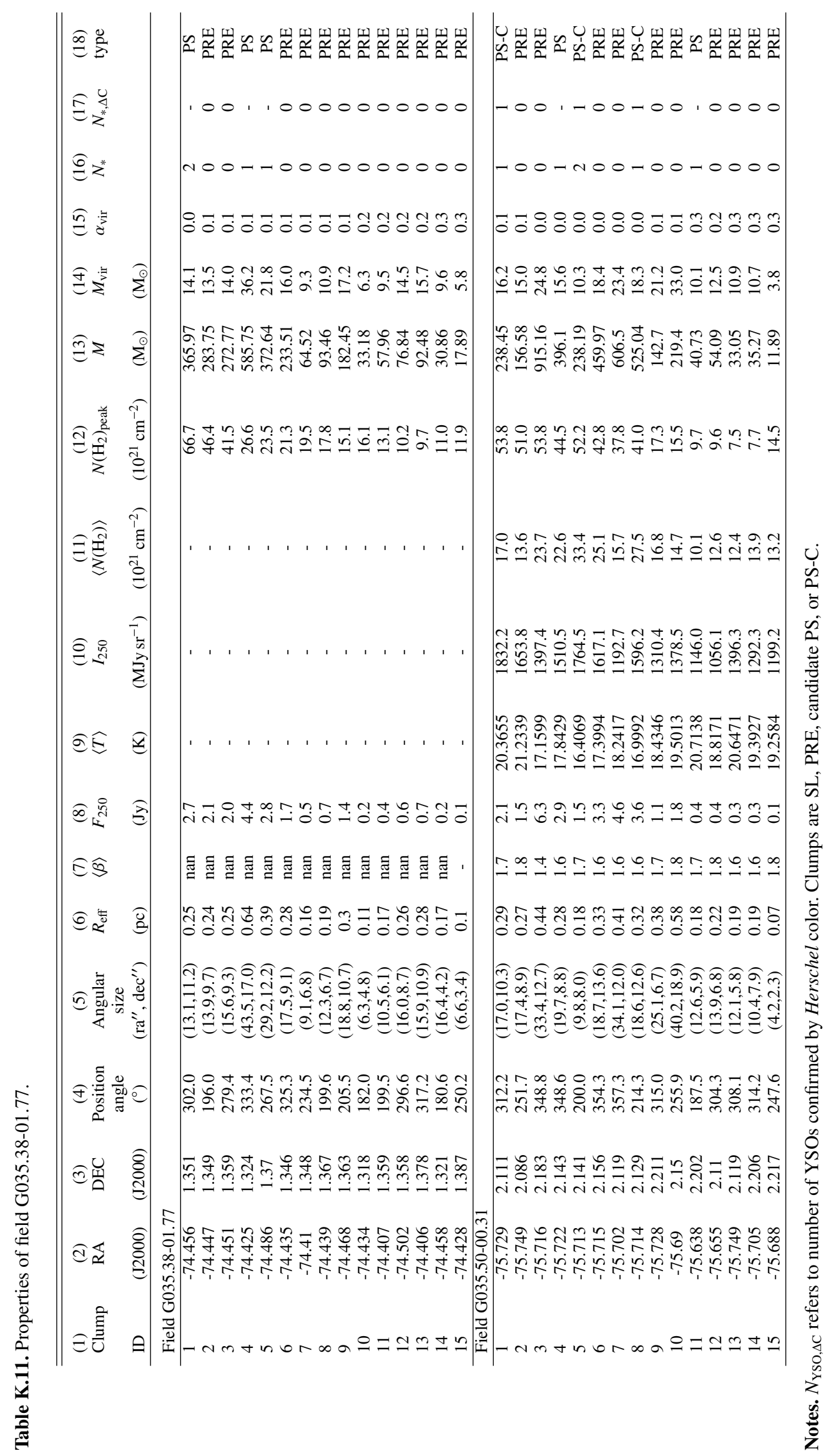


E. Mannfors et al.: Characterization of dense Planck clumps observed with Herschel and SCUBA-2

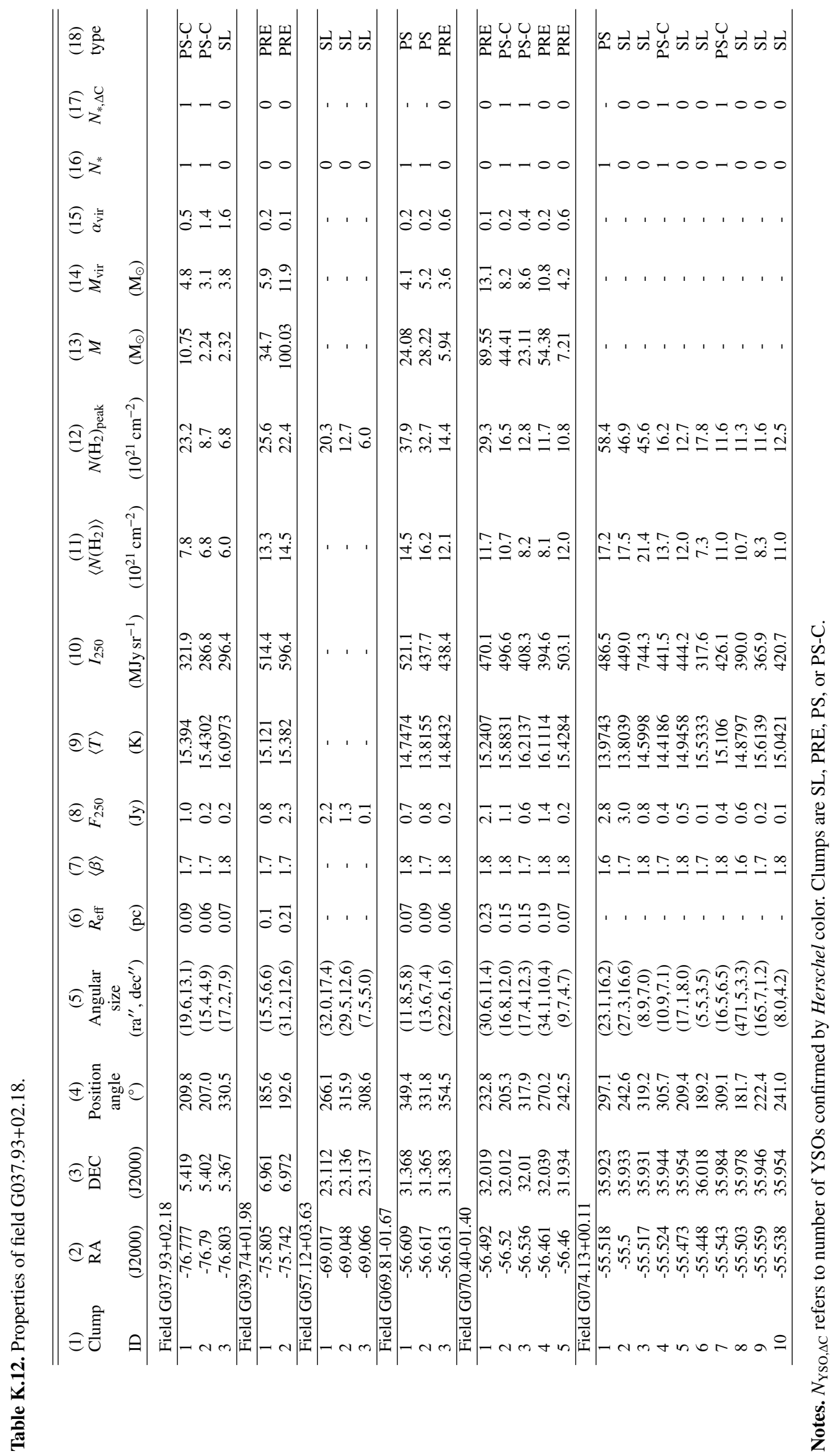




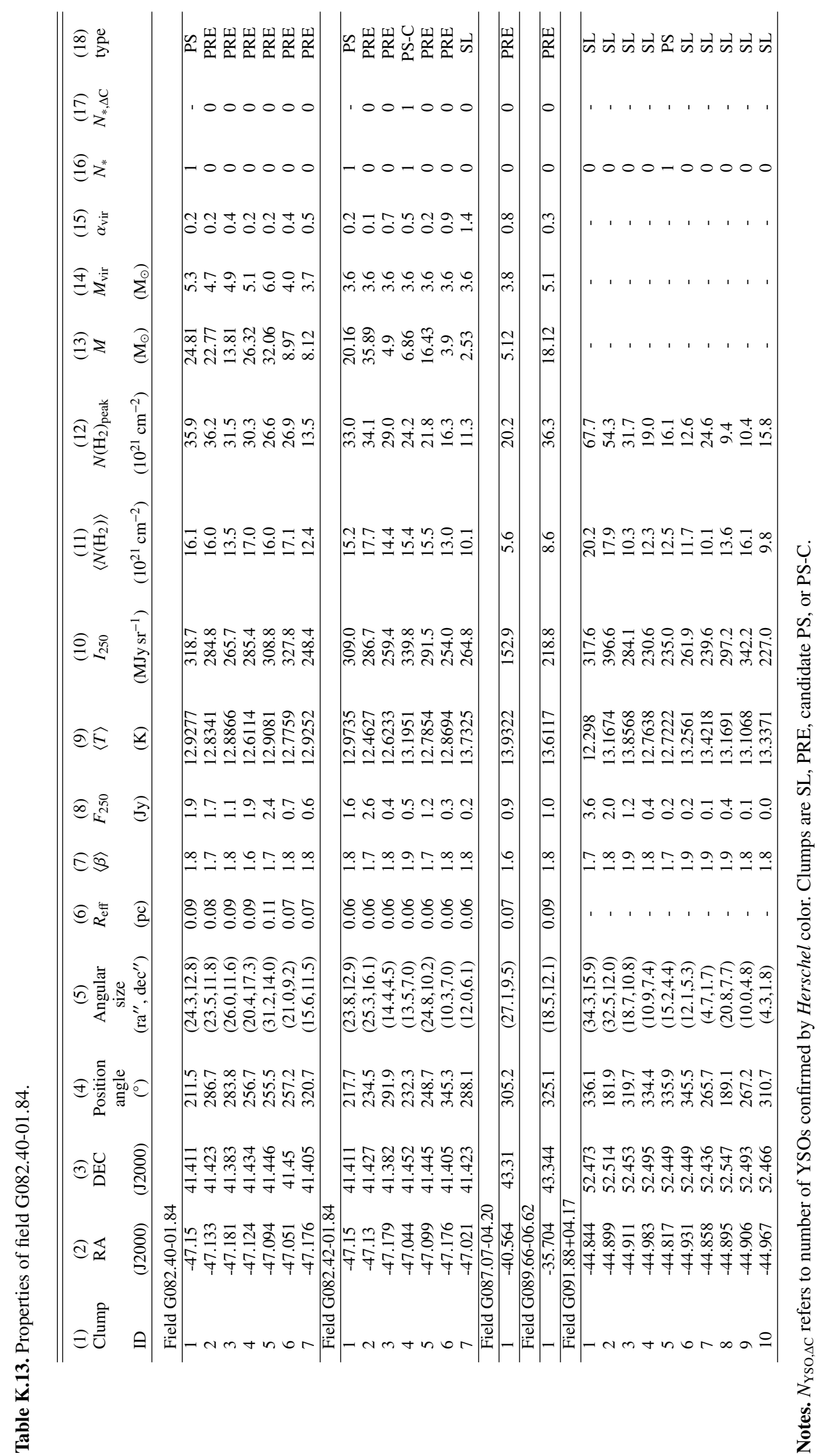


E. Mannfors et al.: Characterization of dense Planck clumps observed with Herschel and SCUBA-2

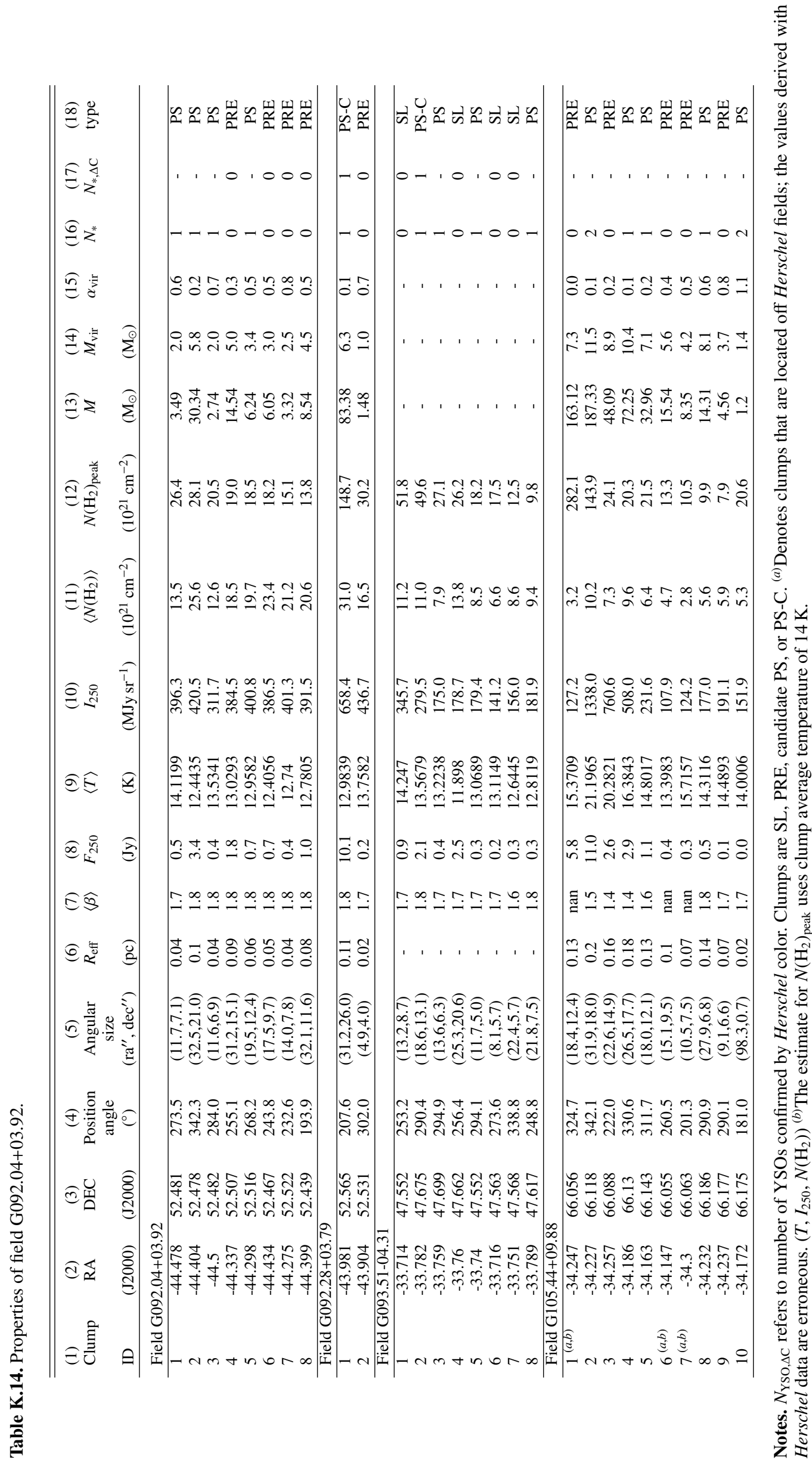




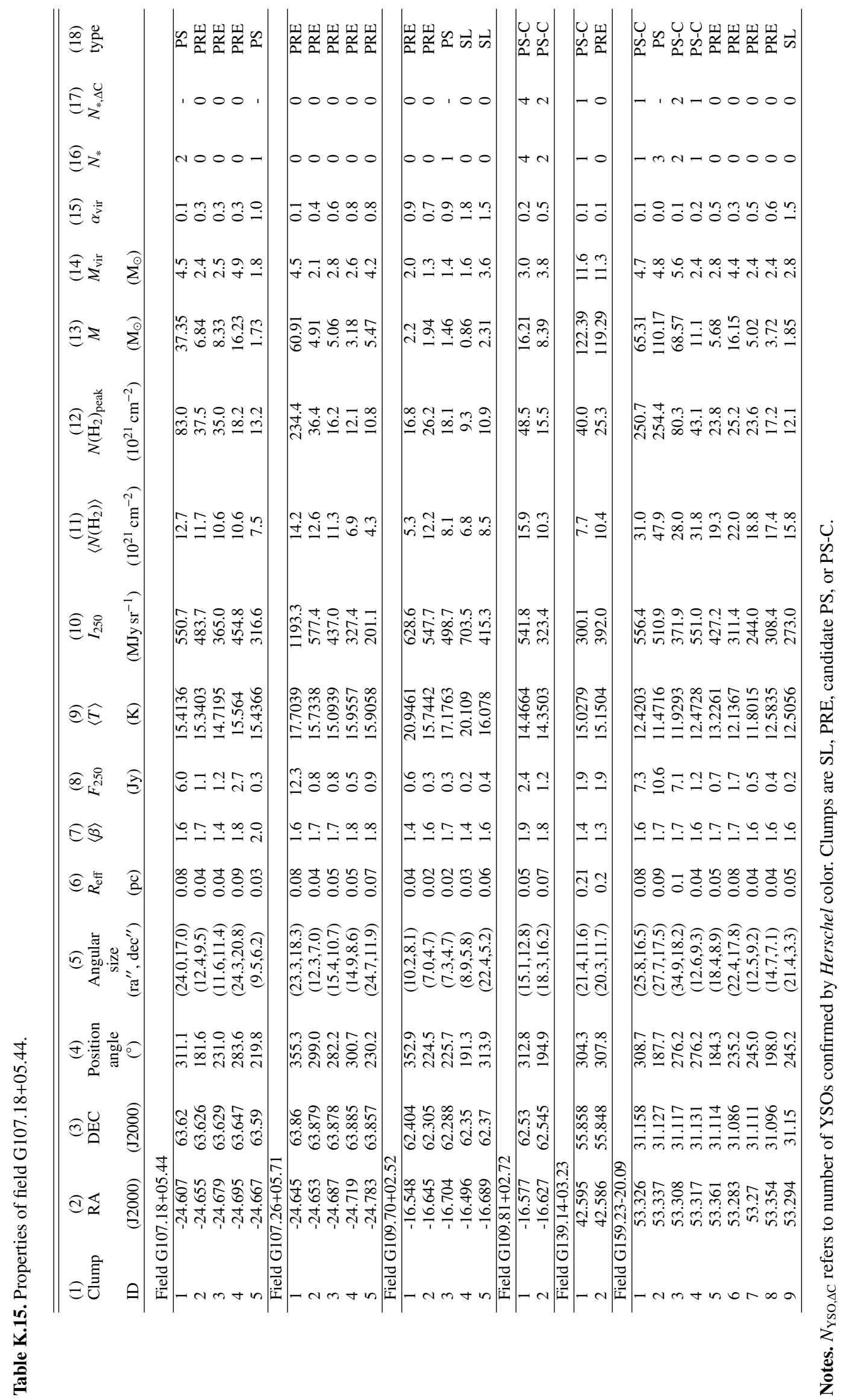


E. Mannfors et al.: Characterization of dense Planck clumps observed with Herschel and SCUBA-2

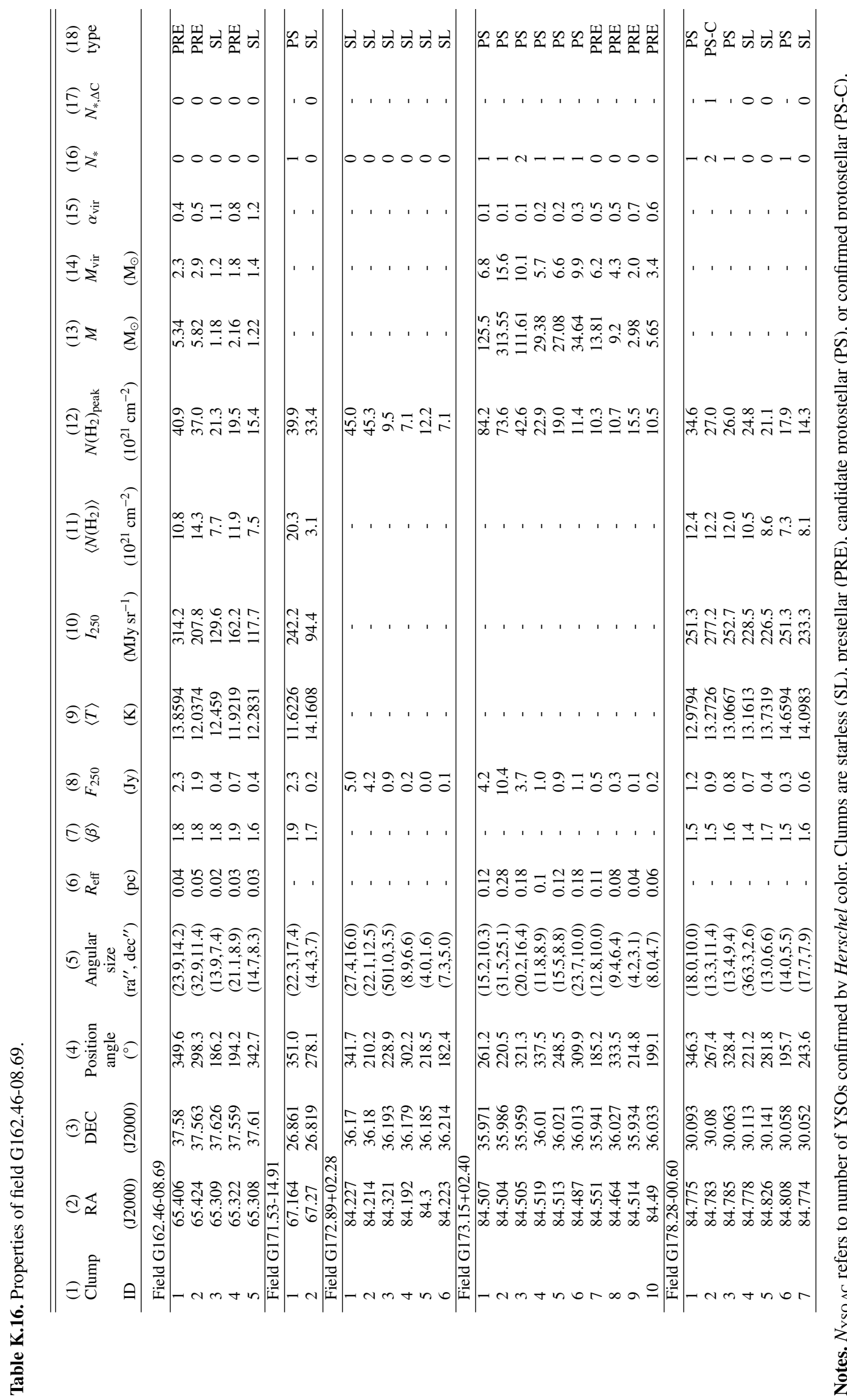




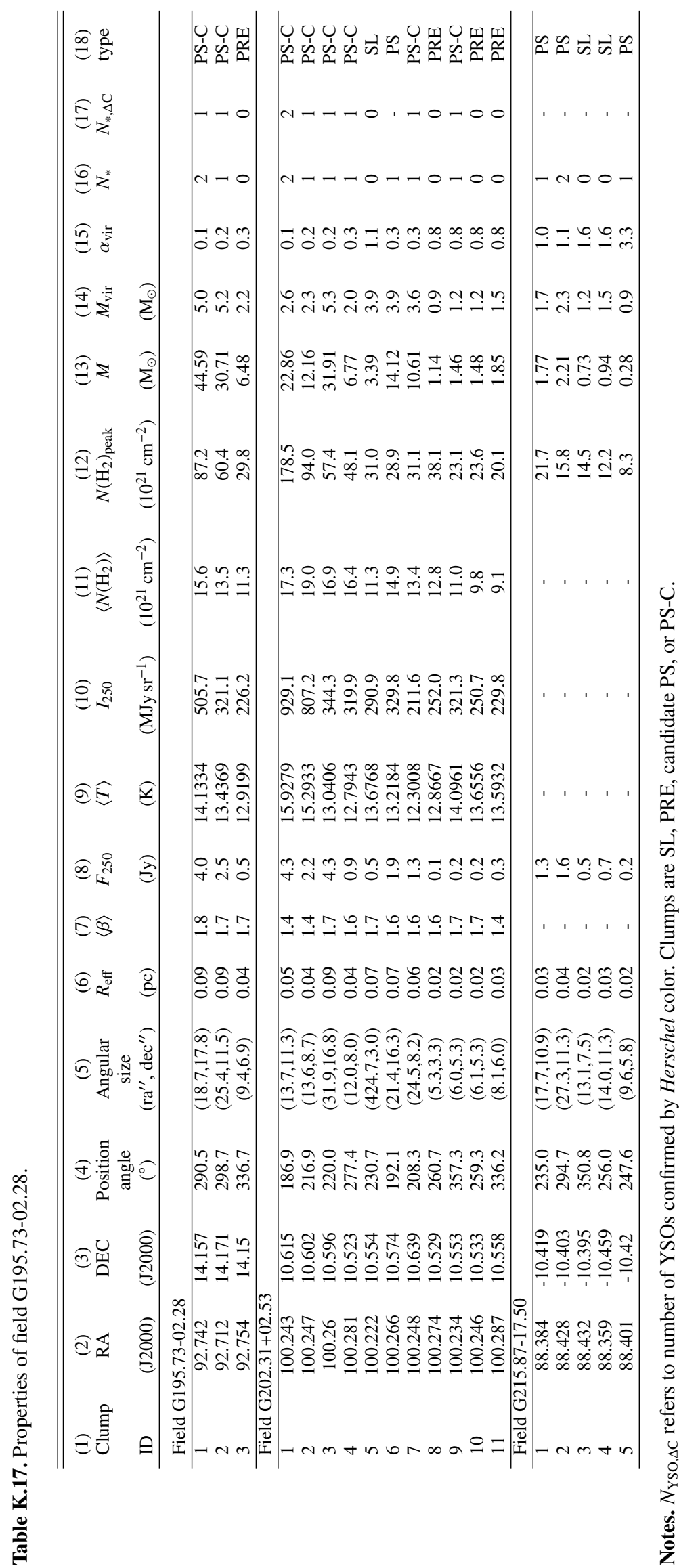


E. Mannfors et al.: Characterization of dense Planck clumps observed with Herschel and SCUBA-2

\section{Appendix L: ${ }^{13} \mathrm{CO}$ line graphs for individual \\ clumps}

The ${ }^{13} \mathrm{CO}$ spectra of the 13 clumps in the fields G105.44+09.88, G159.23-20.09, and G202.31+02.53 are shown in Figs. L.1-L.3. The radial velocity of the peak emission $v_{0}$ and the line widths $\Delta V$ are listed in the frames.
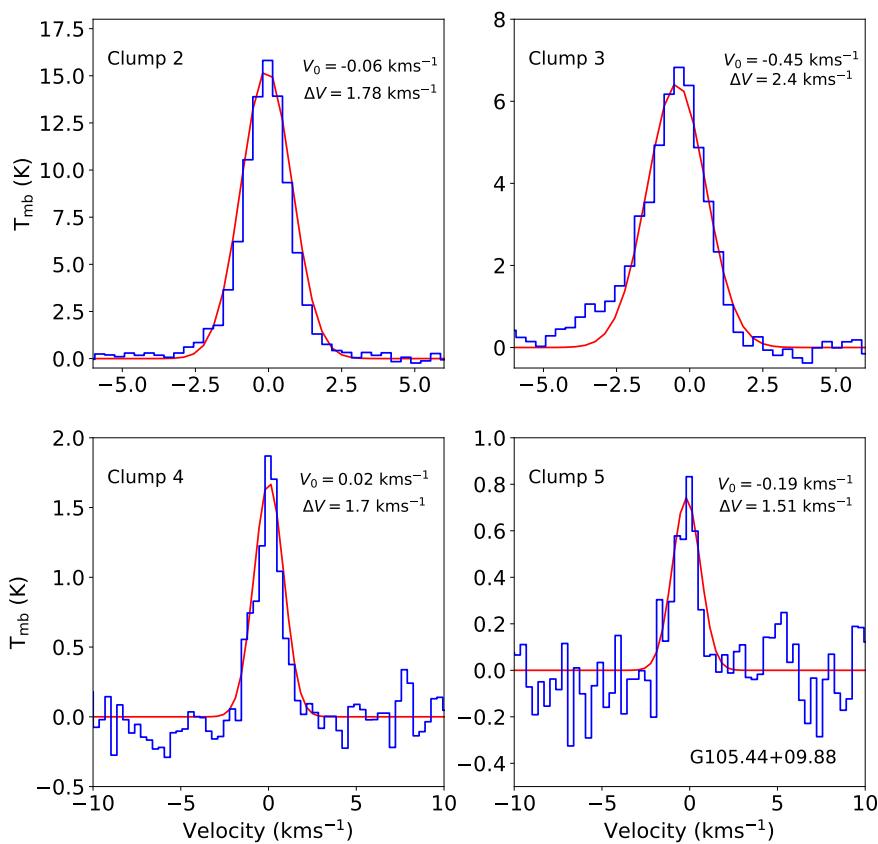

Fig. L.1. ${ }^{13} \mathrm{CO}$ spectra for clumps 2, 3, 4, and 5 in field G105.44+09.88.
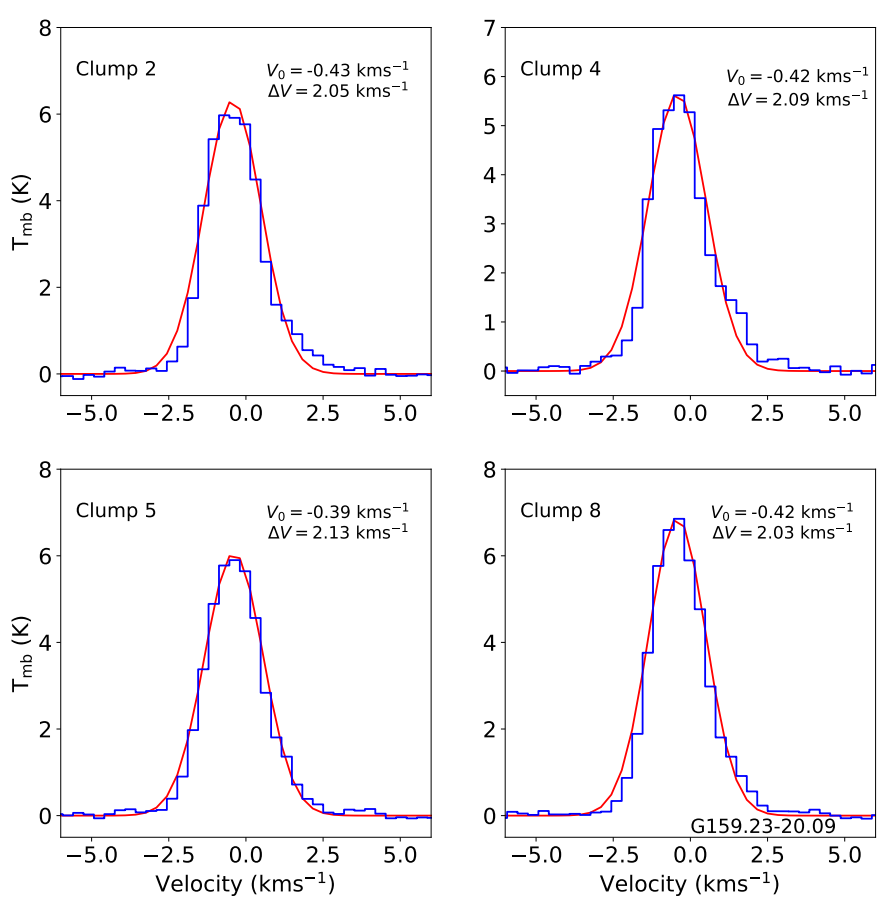

Fig. L.2. ${ }^{13} \mathrm{CO}$ spectra for clumps 2, 4, 5, and 8 in field G159.23-20.09.
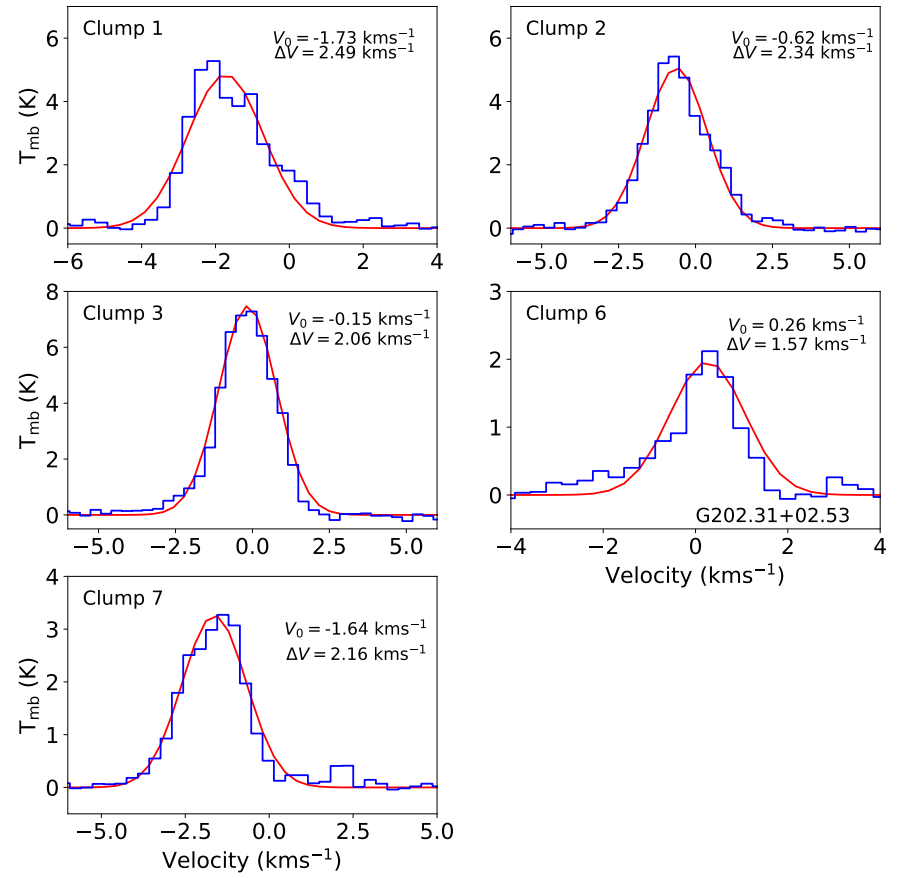

Fig. L.3. ${ }^{13} \mathrm{CO}$ spectra for clumps 1, 2, 3, 6, and 7 in field $\mathrm{G} 202.31+02.53$. 


\section{Appendix M: Clump data}

In this appendix,we show further plots for the clump properties. Figure M.1 shows a correlation plot including the clump distances, aspect ratios, masses, temperatures, column densities, and volume densities. Figures M.2-M.7 show histograms for the individual clump properties, categorized according to the estimated source distances.
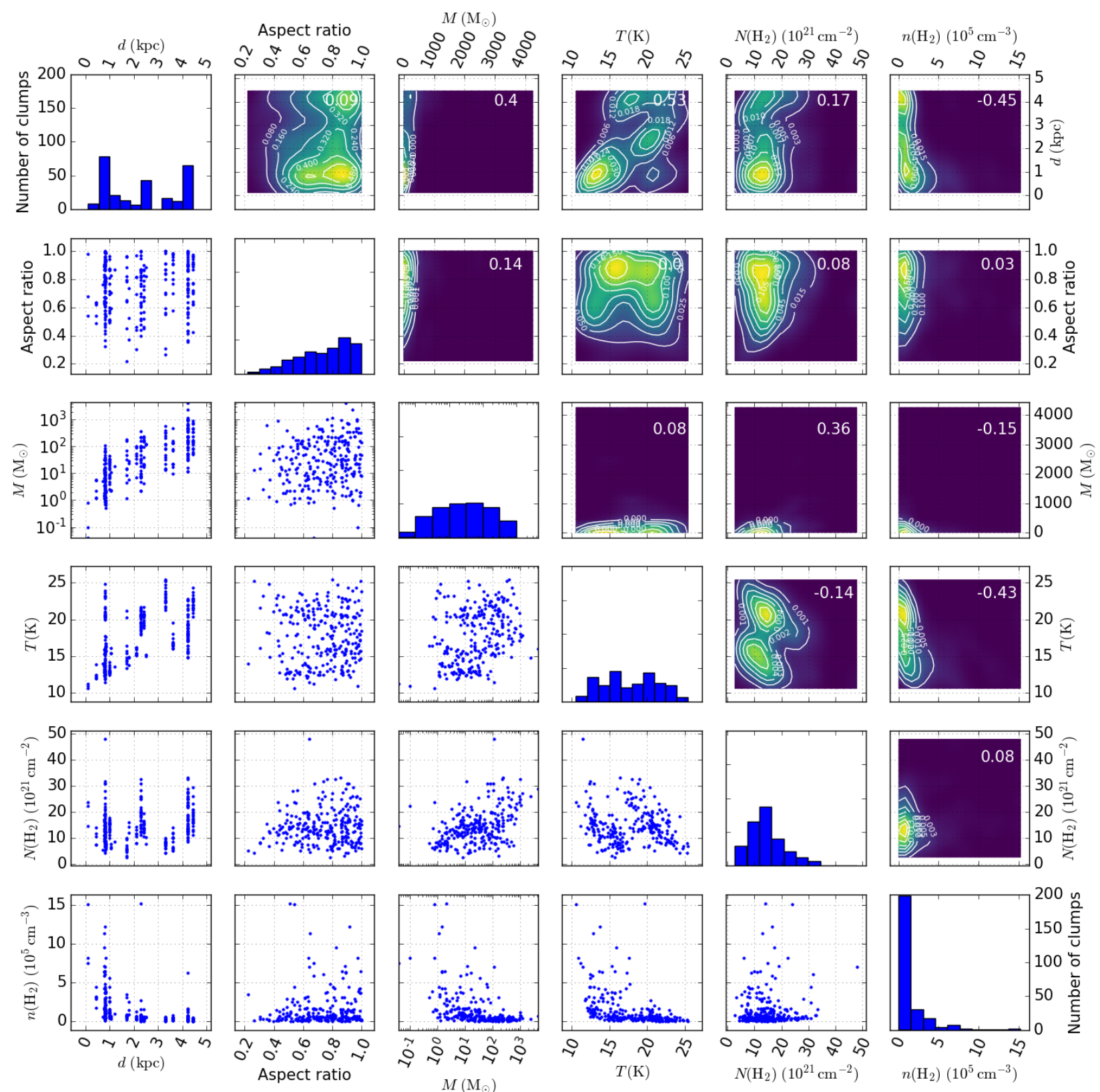

Fig. M.1. Histograms of clump distance, aspect ratio (minor/major axis), mass, temperature, column density $N\left(\mathrm{H}_{2}\right)$, and number density $n$ are shown on the diagonal, with scatter plots relating each parameter to each other on the lower frames. The upper frames show a contour plot of the same relation; the correlation coefficient is quoted in the frame. These values are derived using SPIRE data with constant $\beta=1.8$. In this figure, 242 clumps with distance estimates and SPIRE data are included. Mass has a logarithmic scale for only the histogram and scatter plots. 
E. Mannfors et al.: Characterization of dense Planck clumps observed with Herschel and SCUBA-2
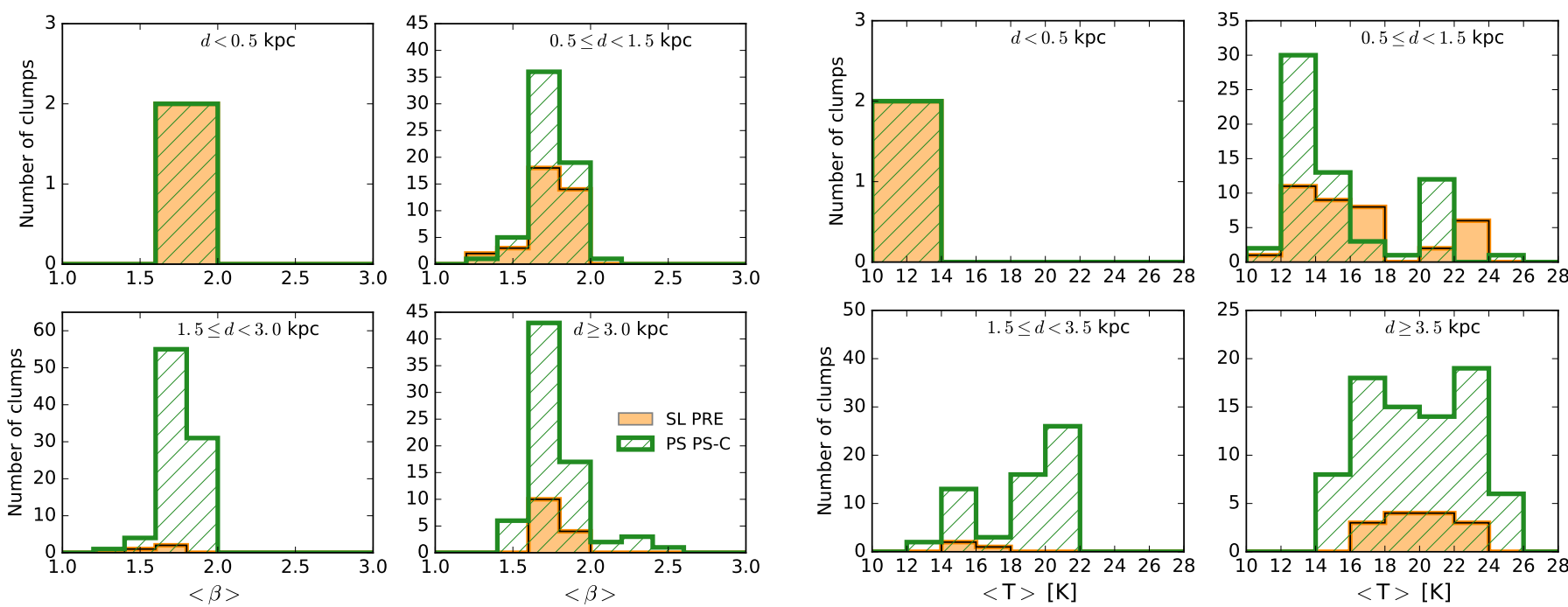

Fig. M.2. Histograms for mean spectral index of all starless (SL PRE), and all protostellar (PS PS-C) clumps in distance bins of $d<0.5 \mathrm{kpc}$, $0.5 \leq d<1.5 \mathrm{kpc}, 1.5 \leq d<3.0 \mathrm{kpc}$, and $d \geq 3.0 \mathrm{kpc}$, written on the images. Includes all 242 clumps with distance estimates and SPIRE data. The number of clumps in each bin is written in column 11 of Table 5.
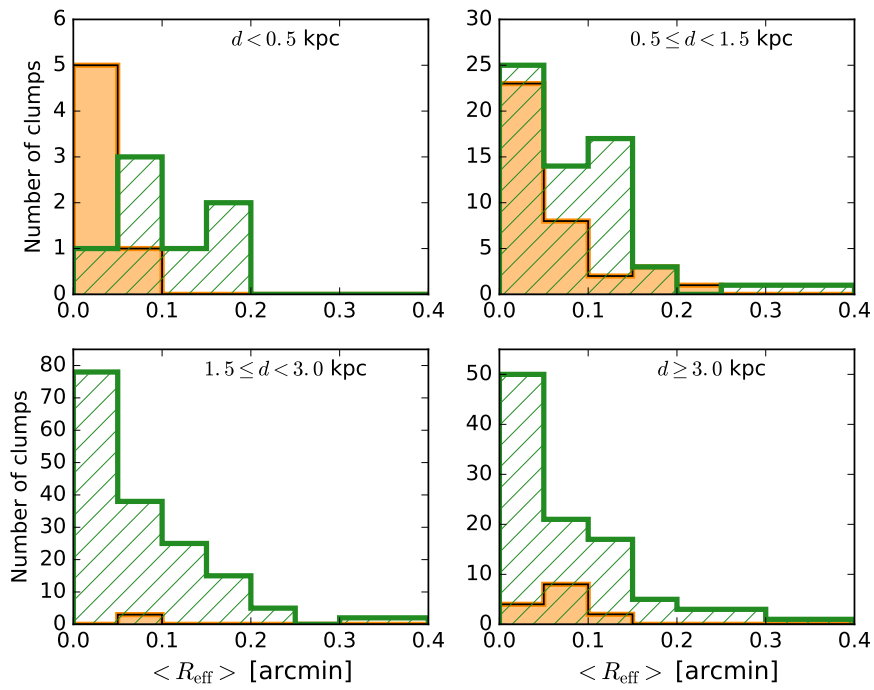

Fig. M.3. Same as Fig. M.2 for mean clump angular effective radius (Eq. (6)). Includes all 336 clumps with distance estimates. The number of clumps in each bin is written in column 11 of Table 5.

Fig. M.4. Same as Fig. M.2 for temperature. The number of clumps in each bin is written in column 10 of Table 5 .
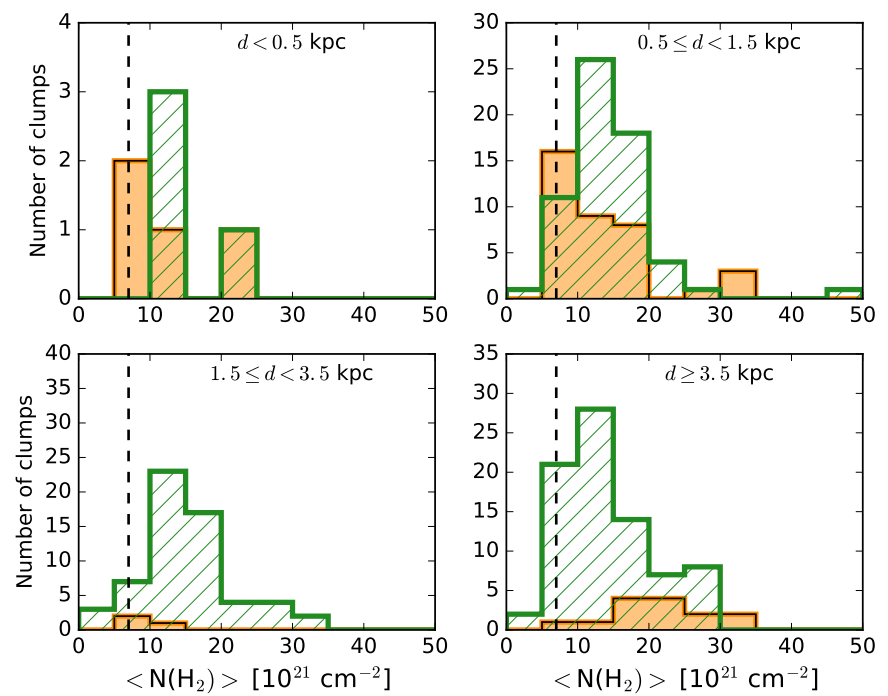

Fig. M.5. Same as Fig. M.2 for mean clump column density. The dotted line corresponds to $A_{\mathrm{v}}=7$. Includes all 242 clumps with distance estimates and SPIRE data. The number of clumps in each bin is written in column 10 of Table 5 . 

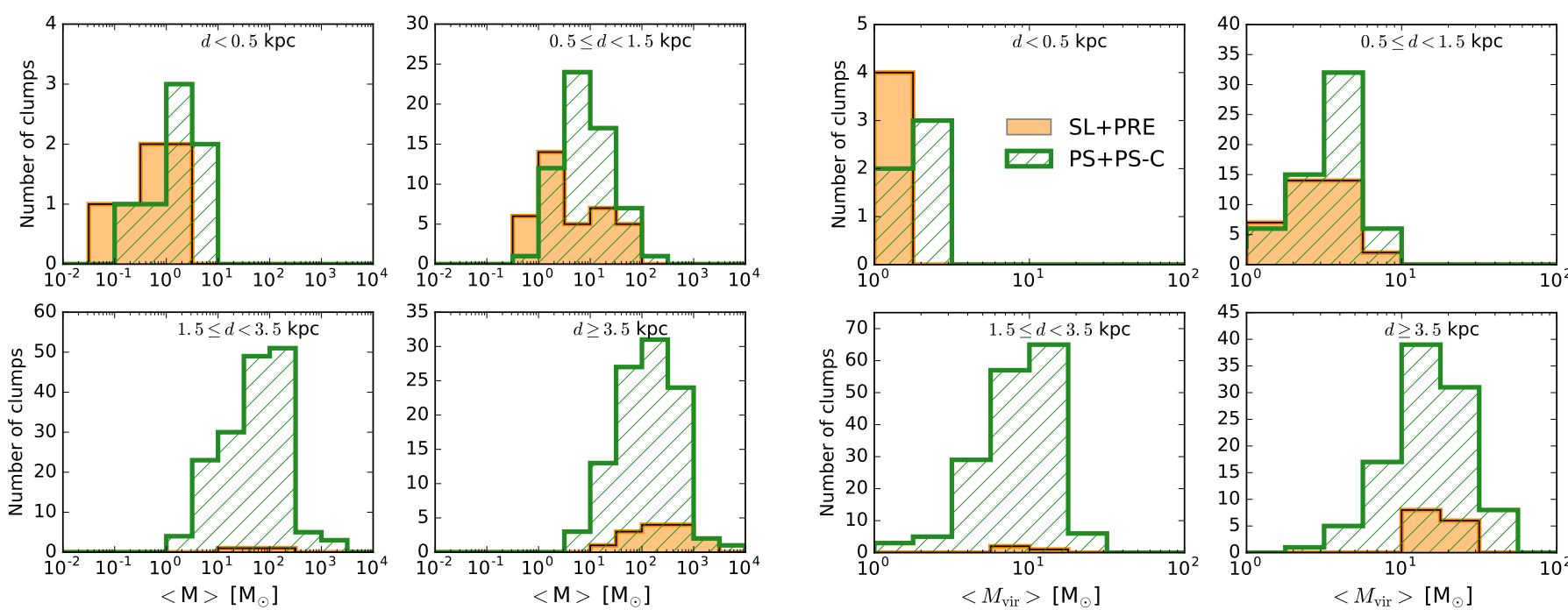

Fig. M.6. Same as Fig. M.2 for clump mass. Includes all 336 clumps with distance estimates. The number of clumps in each bin is written in column 11 of Table 5.
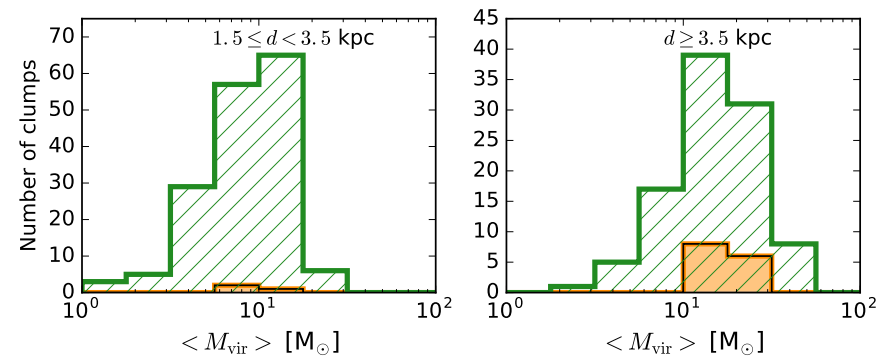

Fig. M.7. Same as Fig. M.2 for clump virial mass. Includes all 336 clumps with distance estimates. The number of clumps in each bin is written in column 11 of Table 5 . 